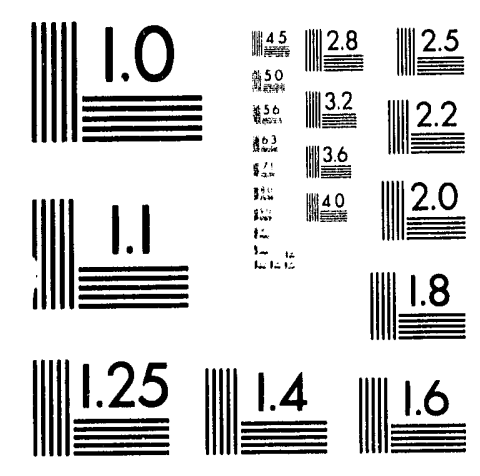



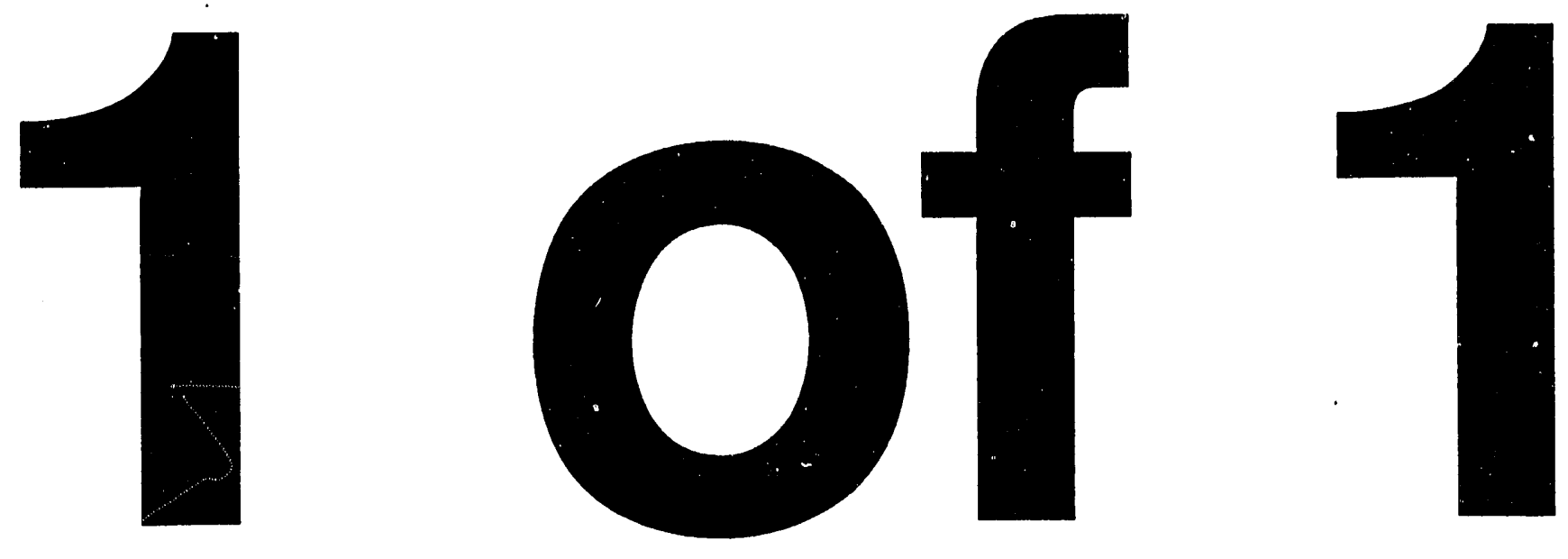


\title{
Transport Effects of Low $(m, n)$ MHD Modes on TFTR Supershots
}

Z. CHANG* ${ }^{\dagger}$, E. D. FREDRICKSON, J. D. CALLEN*, K. M. McGUIRE, M. G. BEL,L, R. V. BUDNY, C. E. BUSH, D. S. DARROW, A. C. JANOS, L. C. JOHNSON, H. PARK, S. D. SCOTT, J. D. STRACHAN, E. J. SYNAKOWSKI, G. TAYLOR, R. M. WIELAND, M. C. ZARNSTORFF, S. J. ZWEBEN and the TFTR group Plasma Physics Laboratory, Princeton University, P. O. Box 451, Princeton, NJ 08543, USA,

*University of Wisconsin, Madison, WI 53706-1687, USA

\begin{abstract}
Supershots in TFTR often suffer a performance deterioration characterized by a gradual decrease of the D-D fusion neutron yield and plasma stored energy after several hundred milliseconds of auxiliary heating. The correlation between this performance deterioration and the development of low $m$ (the poloidal mode number), $n$ (the toroidal mode number) MHD modes is studied through shot-to-shot comparisons and statistical data analyses. A good correlation is observed between performance deterioration and the appearance of strong $3 / 2$ and $4 / 3$ macroscopic modes (magnetic islands) in small major radius plasmas $(R=2.45 \mathrm{~m})$. The magnetic island structures are observed using Mirnov and ECE diagnostics. The measured $T_{e}, T_{i}$ and $n_{e}$ profiles show that development of the islands corresponds to a nearly constant decrement of these quantities over the core region $r<r_{s}$, where $r_{s}$ is the mode rational surface, on a transport time scale $\left(t>\tau_{E}\right)$. The observed energy deterioration scaling, $\delta W / W \sim w / a$, where $w$ is the magnetic island width, agrees with both a local transport model and predictive numerical simulations. For larger major radius plasmas $(R=2.52,2.60 \mathrm{~m})$, a continuous increase of edge recycling rate during the neutral beam injection phase scems to have a larger effect on the performance deterioration than does the MHD.

$\dagger$ Present address: Plasma Physics Laboratory, Princeton University, P. O. Box 451, Princeton, NJ 08543, USA.
\end{abstract}

1

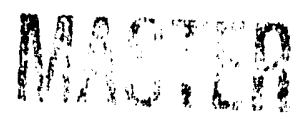




\section{INTRODUCTION}

The supershot [1] in TFTR is a high performance operation regime for fusion plasma physics studies in tokamak devices. It is characterized by its high D-D fusion neutron yiel.d $\left(S_{n}\right)$, high plasma stored energy $(W)$, high ratio of $\tau_{E} / \tau_{E}^{L-\text { mode }}$ ( $\tau_{E}$ is the plasma energy confinement time, and $\tau_{E}^{L-\text { mode }}$ is the Goldston L-mode confinement time [2]), high ratio of $T_{i} / T_{e}$ (temperature ratio for ions and electrons), and highly centrally peaked plasma profiles (density, ion temperature, D-D fusion neutron yield, etc.). Unfortunately, like other recently observed high perfor: mance modes (hot-ion H-mode and PEP H-mode in JET [3], VH-mode in DIII-D [4], and high- $\beta_{p} \mathrm{H}$-mode in JT-60U [5]), the TFTR supershot often suffers a performance deterioration - gradual decrease of the global plasma parameters, such as the total D-D fusion neutron yield, plasma stored energy and the energy confinement time after several hundred milliseconds of high power auxiliary heating. This kind of performance deterioration is observed even in plasmas which do not suffer from other known drastic events such as Carbon blooms [6] and major or minor disruptions.

The performance deterioration in TFTR is a complicated physics phenomenon. Since it usually has a long time evolution phase $\left(t>\tau_{E}\right)$, the plasma behaves as a dissipative non-equilibrium dynamic system. Many internal and external physical processes are involved. This paper will study one of the important mechanisms - low $(m, n)$ MHD (Magnetohydrodynamics) modes (where $n$ and $m$ denote the toroidal and poloidal mode number). The paper is organized as follows. Section 2 describes the basic observation and the characteristics of the correlation between the existence of the low $(m, n)$ MHD modes (primarily the $m / n=2 / 1,3 / 2,4 / 3$ modes) and the performance deterioration. Both shot-to-shot comparisons and statistical analyses of a large number of supershots from the TFTR 1992 run period are presented. Section 3 summarizes the experimental observations of the magnetic island-type structure corresponding to the low $(m, n)$ MHD modes and shows how the plasma parameters evolve when the MHD modes develop. A theoretical model based on a local transport assumption is introduced in Section 4 to describe the physics of the island-induced plasma transport effects. Its predictions are compared with TFTR experiments. In Section 4 numerical calculations are used to simulate the dynamical features of the island-induced performance deterioration. 
The appearance of the MHD modes is not the only mechanism that correlates with the performance deterioration, as will be discussed in Section 2. For larger major radius plasmas the changes in the plasma recycling rate seem to play a more important role. A brief discussion of the possible effects of changes in the edge recycling is given in Section 5. Finally, the conclusions are summarized in Section 6.

\section{OBSERVATION OF THE LOW $(m, n)$ MHD-CORRELATED PER- FORMANCE DETERIORATION}

A correlation between supershot performance deterioration and the existence of low $(m, n)$ coherent MHD modes has been observed [7] for many years in TFTR supershot plasmas. However, due to the lack of highly reproducible, high performance supershots, the MHD effects on supershot performance were often obscured by changes in other plasma parameters or physical processes, such as the large increase of the edge recycling rate often observed during the performance deterioration phase. The situation has been improved in the 1992 TFTR run. In addition to the improvement in many diagnostic systems, a great deal of effort has been dedicated towards achievement of reproducible high performance supershots [8]. Among those similar discharges, many kinds of low $(m, n)$ coherent MHD modes were observed. This allows us to undertake a close comparison between the discharges with and without the MHD modes and to do a reliable statistical data analysis [9], which is important since it may provide us with a useful perspective for conducting future tokamak experiments.

\subsection{Shot-to-shot comparison}

A comparison of three sequential, neutral bearn heated supershots is shown in Fig. 1. They have nearly identical plasma size (major radius $R=2.45 \mathrm{~m}$, minor radius $a=0.80 \mathrm{~m})$, beam power $(24 \mathrm{MW})$, toroidal magnetic field $(4.8 \mathrm{~T})$, plasma current (1.6 MA), wall conditioning (inferred from Carbon II and $D_{\alpha}$ emission measurements), and Ohmic target density. Despite these macroscopic similarities, the three discharges developed very different MHD activity. (The MHD activity studied in this paper is detected mainly by an array of 30 Mirnov coils [10] and a high resolution 20-channel grating polychromator ECE diagnostic system [11].) Note 
that in Fig. 1 the two discharges which developed $m / n=3 / 2$ or $4 / 3$ modes suffered a performance deterioration (i.e., the fractional decrease from the peak value to the value near the end of NBI). The discharge which suffered no deterioration did not have a continuous coherent MHD mode, except for a bursting, fishbone-type $(m / n=1 / 1)$ mode which started at about $3.35 \mathrm{sec}$ (see Section 3.4 for a detailed discussion). The development of the $3 / 2$ mode correlates with a deterioration of $\sim 30 \%$ in the neutron yield and $\sim 15 \%$ in stored energy. This is a larger deterioration than in the case of the $4 / 3$ mode which correlates with a $\sim 25 \%$ drop in neutron yield and a $\sim 10 \%$ drop in stored energy. Corresponding changes are also observed in other plasma parameters (Fig. 2), such as the central electron density $\left(n_{e}\right)$ obtained from the Multichannel Infrared Interferometer (MIRI) [12], central electron temperature $\left(T_{e}\right)$ from the Michelson interferometer system [13] and the core ion temperature $\left(T_{i}\right)$ from the Charge Exchange Recombination Spectroscopy (CHERS) [14]. It is important to emphasize the similarity of the three discharges before the onset of MIID as well as the similarity of the wall conditioning during the entire NBI phase as inferred from the $C_{I I}$ and $D_{\alpha}$ emissivity measurements. These similarities suggest that the performance deterioration observed in these discharges is due mainly to the development of the coherent MHD modes within the plasma and not from changes in edge recycling.

A close correlation in time between the appearance of the $3 / 2$ or $4 / 3$ modes and the decay of the plasma parameters can also be seen from Fig. 1. The time correlation can be seen more clearly in Fig. 3 where two different discharges which display performance deterioration have dominant 3/2 MHD modes that developed at different times. It is clear that the later onset of the mode corresponds to a later start of the deterioration. A similar situation is seen for the $4 / 3$ mode cases shown in Fig. 4. A more severe deterioration correlated with a strong $m / n=2 / 1$ mode has also been observed during the 1992 TFTR run with a larger plasma major radius $\left(R_{0} \simeq 2.61 \mathrm{~m}\right.$ ). Similar phenomenon in the $R=2.45 \mathrm{~m}$ supershot plasmas has also been observed in previous runs [7]. Figure 5 shows two otherwise similar discharges, one with no significant MHD, the other with a strong $2 / 1$ mode. In this case drops of $\sim 60 \%$ in the neutron yield and $\sim 30 \%$ in total stored energy are observed for the $2 / 1$ mode discharge.

The appearance of coherent MHD modes in supershot plasmas appears to be 
unpredictable, although many operational plasma parameters are known to correlate with MHD activity, including wall conditioning and edge safety factor $q_{a}$. Thus, statistical data analysis is used to determine the probability of MHD modes and their effects on plasma performance.

\subsection{Statistical data analysis}

More than 500 neutral beam heated supershot discharges from the TFTR 1992 run period. To restrict the analysis to high performance discharges, several constraints were applied:

(1) Beam power $P_{B}>10 \mathrm{MW}$, constant during the heating pulse.

(2) $\tau_{E} / \tau_{E}^{L-\text { mode }}($ Goldston $)>1.5$.

(3) Beam duration 0.8-1.0 sec.

(4) No major or minor disruptions.

(5) No strong impurity influx.

In the following data analysis we divide the database into three groups according to the plasma major radius.

\subsection{1. $R=2.45 \mathrm{~m}$ supershots}

Figures 6 and 7 show the neutron yield deterioration for a collection of 148 neutral beam heated supershots with plasma major radius $R=2.45 \mathrm{~m}$, minor radius $a=0.80 \mathrm{~m}$ (the plasma touches the inner bumper limiter). Using the constraints listed above and restrictions on the plasma current $I_{p} \simeq 1.60 \mathrm{MA}$ and toroidal magnetic field $B_{t} \simeq 4.8 \mathrm{~T}$ (to emphasize the similarity of the discharges), this subset database covers: beam power $P_{B} \sim 11-25 \mathrm{MW}$ and at the time of peak neutron yield, $S_{n} \sim(1.3-3.1) \times 10^{16} \mathrm{~s}^{-1}, \tau_{E} / \tau_{E}^{L-m o d e} \sim 1.7-2.7, \tau_{E} \sim$ $0.11-0.19 \mathrm{sec},\left\langle\beta_{p}\right\rangle \sim 0.70-1.25, \beta_{N}\left(\equiv \beta_{t}(\%) a B_{t} / I_{p}\right) \sim 1.4-2.1, q_{a} \simeq 4.6-5.1$, $n_{e 0} \sim(4.2-7.7) \times 10^{19} \mathrm{~m}^{-3}, n_{e 0} /\left\langle n_{e}\right\rangle \sim 1.7-2.9, T_{e 0} \sim 7-10 \mathrm{keV}, T_{i 0} \sim 30 \pm 5 \mathrm{keV}$.

The performance deterioration in neutron yield is plotted in Fig. 6, where we have defined

$$
\frac{\delta S_{n}^{\prime}}{S_{n}} \equiv \frac{S_{n}\left(t_{b m-o f f}\right)-S_{n}\left(t_{p k}\right)}{S_{n}\left(t_{p k}\right)} .
$$

Here, $t_{b m-o f f}$ is the time near the beam turn off and $t_{p k}$ is the time at peak neutron yield. In most cases studied, the NBI phase has a one-second duration, from 3.0 
to $4.0 \mathrm{sec}$. We take $t_{b m-o f f}$ to be $\sim 50 \mathrm{~ms}$ before the end of the NBI phase to avoid the small neutral beam power drop at the end of the NBI phase in some discharges. So if the neutron yield keeps increasing during the entire NBI phase, $\delta S_{n} / S_{n}$ will become positive. A similar expression is used for the stored energy deterioration $\delta W / W$ in the following analysis. In this case, $t_{p k}$ is the time at the peak of the stored energy, which is usually $\lesssim 50 \mathrm{~ms}$ later than the peak neutron yield time. The following statistics are observed:

(1) Performance deterioration statistics

Long duration, high performance was sustained for most of the supershots: Namely, 108 discharges $(73 \%)$ have $\delta S_{n} / S_{n}<10 \%$. Only about 40 discharges $(27 \%)$ have $\delta S_{n} / S_{n}>10 \%$. Of these, 28 discharges $(\leqslant 20 \%)$ displayed significant neutron yield deterioration with $\delta S_{n} / S_{n}>15 \%$. Figure 7 shows that the amount of deterioration increases with neutral beam power. In the discharges with $P_{B}<$ $14 \mathrm{MW}$ no deterioration was observed. The deterioration in stored energy (Fig. 8) satisfies a relation $\delta W / W \simeq(1 / 2) \delta S_{n} / S_{n}$, which agrees with the quadratic scaling $S_{n} \propto W^{2}$ previously reported for TFTR supershots [15].

(2) MHD statistics

Of the 148 discharges, 16 discharges $(\sim 10 \%)$ developed a dominant $m / n=3 / 2$ mode, typically near the time of peak performance (in neutron yield and stored energy). A dominant $m / n=4 / 3$ mode developed in 28 discharges ( $\leqslant 20 \%$ ). A distinct coherent $m / n=5 / 4$ mode was detected in $\sim 11$ discharges $(\sim 7 \%)$. These coherent MHD modes usually do not co-exist. Once excited, they tend to persist through the rest of the NBI phase. More than half of the discharges ( $\sim 77$ discharges) have fishbone-type $(q=1)$ bursting MHD modes. There are 16 discharges $(\sim 10 \%)$ which are "MHD quiet", which means no coherent MHD modes were detected by either the Mirnov or ECE diagnostics. In many cases some edge kink-like $(m / n=5 / 1,4 / 1$ or $3 / 1)$ MHD activity was detected in coexistence with the dominant MHD modes listed above. No $m / n=2 / 1$ modes were detected in this $I_{p} \simeq 1.6 \mathrm{MA}$ subset database.

Although Fig. 7 seems to suggest a low probability of the $3 / 2$ or $4 / 3$ MHD activity in low $P_{B}(<16 \mathrm{MW})$ discharges, the lack of enough statistical data in this region weakens this argument. The appearance of the coherent MHD modes does not seem to be closely dependent on the global $\beta$ value (or nearness to the 
Troyon limit [16]). As can be seen from Fig. 9, a lot of coherent MHD activity has already been observed in the lower (comparing with the Troyon limit) $\beta_{N}$ discharges. For example, among the 30 discharges that have $\beta_{N}=1.5-1.6$ or $\beta_{N} / \beta_{c}^{\text {Troyon }} \sim 70 \%, 10$ discharges $(30 \%)$ developed $3 / 2$ or $4 / 3$ modes. This statistics is about the same for the higher $\beta_{N}$ case, e.g., in the group of another 30 discharges with $\beta_{N}=1.9-2.0$ or $\beta_{N} / \beta_{c}^{\text {Troyon }} \sim 90 \%, 10$ of them (30\%) developed $3 / 2$ or $4 / 3$ modes.

(3) Correlation between $M H D$ and performance deterioration

Among the 40 discharges that have $\delta S_{n} / S_{n}>10 \%$, we find that 34 discharges have either $3 / 2$ or $4 / 3$ modes. All but one of the 28 discharges with $\delta S_{n} / S_{n}>15 \%$ have a rotating $3 / 2$ or $4 / 3$ MHD mode. The drop in neutron yield associated with a $3 / 2(4 / 3)$ mode can be up to $\sim 30 \%(\sim 25 \%)$ (see Fig. 8$)$, and the corresponding $\tau_{E}$ drop (between the time of the peak neutron yield and the end of NBI phase) is $\sim 25 \%$ (20\%), where the measurement is taken between the time of peak neutron yield and the end of NBI. Note from Fig. 1 that since the stored energy keeps decreasing in the large deterioration discharges after the onset of the $3 / 2$ or $4 / 3$ MHD modes, we have $\delta \tau_{E} / \tau_{E} \neq \delta W / W$ [where $\tau_{E} \equiv W /\left(P_{i n}-d W / d t\right), P_{\text {in }}$ is the total input power].

Like the MHD-quiet discharges (except the one exceptional discharge 68244), most of the discharges with fishbone-type modes or the $5 / 4$ mode do not exhibit significant performance deterioration (i.e., $\delta S_{n} / S_{n} \leqslant 10 \%$ ).

The above statistics suggest that there is a strong correlation between the existence of the $3 / 2$ and $4 / 3 \mathrm{MHD}$ modes and performance deterioration. Fig. 10 illustrates the correlation between the onset time of the $3 / 2$ and $4 / 3$ modes and the peak neutron yield time. We find that, within the statistical error bars, the peak in $S_{n}$ never precedes the MHD. This is consistent with the conjecture that the MHD causes the performance deterioration. However, the presence of coherent low $(m, n)$ MHD modes is not a necessary condition for the deterioration. Infrequently, performance roll-over is observed in the absence of a strong MHD event. One example is the exceptional discharge (68244) with high neutron yield shown in Fig. 6. As shown in Fig. 11, the neutron yield starts to decrease in this discharge at $\sim 0.5 \mathrm{sec}$ after the NBI. There is no detectable rotating, coherent MHD activity around that time. $\mathrm{A} \sim 16 \%$ deterioration is already observed in the neutron yield 
before the time of an internal crash at $\sim 3.70 \mathrm{sec}$. The reason for this early deterioration is not clear. It may correlate with the small increase in the $C_{I I}$ emissivity and/or the broadening of the electron density profile. Nevertheless, this kind of event occurs much less often than the MHD events in this $R=2.45 \mathrm{~m}$ subset of the supershot database.

The development of low $(m, n)$ MHD modes also correlates with a significant enhancement in the fusion product losses ( $3 \mathrm{MeV}$ proton and $1 \mathrm{MeV}$ Triton) which are measured by a scintillator detector located at the bottom of the vacuum chamber [17]. Figure 12 shows a subset of data from Fig. 6 with $P_{B} \simeq 24 \mathrm{MW}$. The enhancement factor above MHD-quiescent discharges is about $10-20 \%$ for the fishbone-type modes (see a typical discharge in Fig. 22), a factor of about 2 for the $m / n=4 / 3$ modes, and a factor of about 3 for the $m / n=3 / 2$ modes [17]. The MHD-quiescent level of about 10 units on this scale in Fig. 12 is composed of first-orbit and "delayed loss" [17]. A fast saturation of the neutron-normalized, MHD-induced loss is observed as the $\log \left(\tilde{B}_{\theta m, n}\right)$ increases, where the $\tilde{B}_{\theta m, n}$ is the edge magnetic fluctuation of the dominant coherent MHD modes measured by Mirnov coils. This scaling is true for both this set of discharges at $0.7 \mathrm{sec}$ after NBI [Fig. 12(a)] and for the time-dependence of the loss versus the $\tilde{B}_{\theta}$ for a single discharge, Fig. 12(b). This seems to imply that the fusion product population susceptible to MHD-induced loss is depleted at MHD levels well below the largest found in the experiment. The loss of beam ions $\left(E_{b} \sim 100 \mathrm{keV}\right)$, on the other hand, is not well diagnosed in TFTR supershot operation regime. It is not clear how many beam ions were lost during the MHD-correlated performance deterioration.

\subsection{2. $R \simeq 2.52 \mathrm{~m}$ supershots}

The supershot database with major radius $R \simeq 2.52 \mathrm{~m}$ is relatively small ( $\sim$ 56 discharges) in the TFTR 1992 run, under the constraints given in Section 2.2. The performance deterioration is plotted in Fig. 13. The parameter ranges are: $R=2.50-2.55 \mathrm{~m}, I_{p}=1.6-2.5 \mathrm{MA} P_{B}=18-28 \mathrm{MW}, B_{t} \simeq 4.6,5.1 \mathrm{~T}$, and at the time of peak neutron yield: $S_{n} \sim(1.4-3.4) \times 10^{16} \mathrm{~s}^{-1}, \tau_{E} / \tau_{E}^{L-\text { mode }} \sim 1.5-2.6$, $\left\langle\beta_{p}\right\rangle \sim 0.5-1.1, q_{a} \simeq 3.5-5.6, n_{e 0} \sim(5-8) \times 10^{19} \mathrm{~m}^{-3}, n_{e 0} /\left\langle n_{e}\right\rangle \sim 1.7-2.9$, $T_{e 0} \sim 8-11 \mathrm{keV}$.

As can be seen from Fig. 13, stronger performance deterioration was seen in 
these discharges than was seen in the $R=2.45 \mathrm{~m}$ plasmas. Out of the 56 discharges, 40 discharges $(\gtrsim 70 \%)$ have neutron yield deterioration $\delta S_{n} / S_{n}>15 \%$, and 30 discharges $(\gtrsim 50 \%)$ displayed $\delta S_{n} / S_{n}>20 \%$.

MHD analysis shows that most of the discharges do not have strong $m \geq 2$ modes. Instead, the $m / n=1 / 1$ type mode and/or fishbone-type bursting mode is the dominant MHD activity in most of the discharges at around the peak neutron time. The correlation between the appearance of the $3 / 2$ or $4 / 3$ MHD modes and the neutron deterioration can still be seen. But this correlation is much weaker statistically compared to the $R=2.45 \mathrm{~m}$ cases. Namely, at $R \simeq 2.52 \mathrm{~m}$ there are more discharges that do not have strong $3 / 2$ or $4 / 3$ modes but displayed significant performance deterioration. A large deterioration is observed even in the "MHD quiet" discharges in which no coherent mode or structures are detected with the Mirnov or ECE diagnostic systems. This implies that there are other physical mechanisms correlated with the performance roll-over in the larger size plasmas. This phenomenon is also seen clearly in the following $R \simeq 2.60 \mathrm{~m}$ supershot analysis.

\subsection{3. $R \simeq 2.60 \mathrm{~m}$ supershots}

There are $\sim 104$ discharges in the 1992 TFTR supershot database with $R \simeq$ 2.60 under the constraints given in Section 2.2. The neutron yicld deterioration analysis is shown in Fig. 14. This set of data covers: $I_{p}=1.4,1.6,1.8 \mathrm{MA} B_{t} \simeq$ 4.5,4.8 T, $P_{B}=12-28 \mathrm{MW}$, and at the time of peak neutron yield: $S_{n} \sim$ $(0.5-3.4) \times 10^{16} \mathrm{~s}^{-1}, \tau_{E}=0.13-0.19 \mathrm{sec}, \tau_{E} / \tau_{E}^{L-\operatorname{mode}} \sim 1.6-2.9,\left\langle\beta_{p}\right\rangle \sim 0.65-1.30$,

$q_{a} \simeq 5.0-8.0, n_{e 0} \sim(3.0-6.5) \times 10^{19} \mathrm{~m}^{-3}, n_{e 0} /\left\langle n_{e}\right\rangle \sim 1.5-2.8, T_{e 0} \sim 6.0-10.5 \mathrm{keV}$.

Similar to the $R=2.52 \mathrm{~m}$ case, the probability of large deterioration is much higher than in the $R=2.45 \mathrm{~m}$ case. Namely, among the 104 supershots, 87 discharges $(\sim 84 \%)$ displayed $\delta S_{n} / S_{n}>15 \%$, and about half of the discharges had $\delta S_{n} / S_{n}>25 \%$.

A group of strong $m / n=2 / 1$ mode discharges were identified in this data set. They correspond to a large neutron yield deterioration - up to $\sim 60 \%$. Also many dominant $3 / 2$ and $4 / 3$ modes are seen in the large deterioration discharges. However, as in the $R=2.52 \mathrm{~m}$ case, there are many discharges that have significant deterioration but do not have correlated strong MHD modes. Most of these 
supershots have "weak MHD" which includes $m / n=1 / 1$ mode, a fishbone-type bursting mode and other weak MHD modes which are not detected in the Mirnov data but may be seen in the ECE data. Disregarding the uncertainty in the mode analysis for these discharges, the statistics show that among the 87 discharges that developed more than $15 \%$ deterioration in neutron yield, only about 38 discharges ( $<50 \%$ ) have strong coherent $m \geq 2$ modes and more than half of the 87 discharges have no discernible MHD. The search for the mechanism responsible for the performance deterioration in larger major radius plasmas is continuing. A discussion of possible effects of edge recycling is given in Section 5.

\section{OBSERVATION OF MAGNETIC ISLANDS AND THEIR EFFECTS ON PLASMA PROFILES}

Magnetic island-type structures have been observed in the $T_{e}(R)$ profiles in supershots with large $m / n=2 / 1,3 / 2$ or $4 / 3$ modes using the 20 channel fast ECE polychromator diagnostic system. In this section we describe the typical features of these tearing-type modes and their effect on the plasma profiles. Also discussed in this section is the effect of the $m / n=5 / 4$ mode and the most commonly observed fishbone-type mode on electron temperature evolution.

\section{1. $m / n=2 / 1$ island}

Large island structures are observed around the $q=2$ rational surface in the $m / n=2 / 1$ mode discharges identified in Fig. 14. Figure 15 shows the electron temperature profiles at two different times - before the MHD and after the MHD. A $\sim 20 \mathrm{~cm}(w / a \sim 0.20$, with $a \simeq 96 \mathrm{~cm})$ magnetic island can be easily identified from the two $T_{e}(R)$ profiles plotted across the $O$ point and $X$ point of the island. It is interesting to note a "bump" structure within the island on the inner side of the $T_{e}$ profile. This structure is consistent with the $T_{e}(R)$ measurement from a high resolution Thomson Scattering system [18], in which bumps on both side islands are observed. (The ECE channel located around the center of the outer side island was not functioning.) A 2-D $T_{e}$ contour plot constructed using the ECE data is presented in Fig. 16. 


\section{2. $\mathrm{m} / \mathrm{n}=3 / 2$ and $4 / 3$ islands}

When a strong $m / n=3 / 2$ or $4 / 3$ mode develops, corresponding magnetic island-type structures are detected in $T_{e}(R)$ from the fast ECE diagnostic. Figure 17 shows the measured $T_{e}(R)$ profile evolution following the development of a strong $m / n=3 / 2$ mode. (This is the same $3 / 2$ mode discharge as shown in Fig. 1.) The $3 / 2$ mode starts after $\sim 3.3 \mathrm{sec}$ when the plasma performance is still improving. At $\sim 3.4 \mathrm{sec}$ the plasma reaches its maximum performance although the energy confinement time has already started to decrease at this time, as shown in Fig. 1. Near the end of the beam phase, a clear $3 / 2$ island structure can be seen in the $T_{e}$ profile [Fig. $17(\mathrm{a})$ ] with a width $w \sim 8 \mathrm{~cm}$, or $w / a \gtrsim 0.10$. The radial variation of the temperature fluctuations is shown in Fig. 17(b). It is interesting to note that even during the early starting up phase of the mode (at $\sim 3.4 \mathrm{sec}$ ) the temperature fluctuation around the mode-rational-surface has already increased to its saturation level. An edge mode with $m / n=3 / 1$ is also seen in this discharge [Fig. 17(c)]. Since this mode also occurs in the discharges with the fishbone-type MIID, for which no severe performance deterioration is obscrved, this mode is not believed to have a direct effect on the plasma profile evolution. In many other $3 / 2$ mode discharges (for example, the discharges shown in Fig. 3) this mode does not occur. Figure 18 shows the time evolution of the electron temperature at different plasma radii. The propagation of the island transport effect to the plasma center seems to take less than $50 \mathrm{msec}$. Then, following the slow increase of the mode amplitude, the $T_{e}$ profile inside the mode rational surface continuously decreases until the mode saturates after $\sim 300$ msec.

A similar $T_{e}$ profile evolution is observed in the discharges with a dominant 4/3 mode. Fig. 19 shows the ECE and Mirnov data. After some fishbone-type MHD bursts, the $4 / 3$ mode starts to grow at $\sim 3.5 \mathrm{sec}$. When the mode is fully developed, a drop of $T_{e}$ inside the mode rational surface results. Outside the mode rational surface $T_{e}$ remains relatively unchanged. The calculated effective island size (see Section 4 ) in this case is small, $\sim 5-8 \mathrm{~cm}$, which is close to the $6 \mathrm{~cm}$ spatial resolution of the diagnostics.

Due to the limited temporal and spatial resolution, the island structures ("flat spots") in $n_{e}$ and $T_{i}$ are less clearly resolved. However, the effect of the island on the evolution of the plasma profiles $\left(n_{e}, T_{e}, T_{i}\right.$ and even the neutron emissivity 
profile $S_{n}$ ) can be observed. This argument is well supported from the statistical data analysis. Shown in Figs. 20(a)-(c) are the relative changes (between the peak neutron yield time and near the end of neutral beam) of plasma parameters near the core versus the relative changes outside the mode rational surface. The data is from the $R=2.45 \mathrm{~m}$ subset database with $P_{B}>20 \mathrm{MW}$. It is clearly seen that the coherent MHD mainly affects the plasma profiles inside the mode rational surface.

\section{3. $m / n=5 / 4$ mode}

A dominant $m / n=5 / 4$ coherent MHD mode has also been observed in TFTR supershots. Due to the higher $n$ and $m$ numbers, this mode has a higher frequency $(\sim 50 \mathrm{kHz})$ and lower $\tilde{B}_{\theta}$ than the $3 / 2$ or $4 / 3$ modes. Shown in Figs. $21(\mathrm{a})$-(c) are the $T_{\epsilon}(R), \delta T_{e} / T_{e}$ and $\dot{B}_{\theta}$ spectrum. This mode does not seem to affect plasma performance, possibly due to its spatial localization around the mode rational surface and small island width (the calculated effective island width $w_{\text {eff }} / a<0.02$, see Fig. 26b).

The observation of various coherent MHD modes in similar discharges described above indicates that the thresholds for exciting these modes must be very similar. Understanding the mechanism that causes the discrete coherent modes is a great challenge to plasma theory. Resistive MHD simulations indicate that a supershot plasma should be more unstable to the $\mathrm{m} / \mathrm{n}=2 / 1$ mode than the other higher $(m, n)$ modes [19]. When finitc $\beta$ stabilizing effects are included, the theory indicates that the plasma may become stable to all the tearing modes [20]. The neoclassical MHD theory [21] may be able to explain why the modes are unstable and their linear growth in time, however the theory predicts not just one but many $(m, n)$ modes present simultaneously. Most other existing theories (e.g., low $n$ kink-ballooning mode [22]) also seem to have difficulty explaining the MHD activity observed in TFTR supershot plasmas. Detailed comparisons of theory and experiment will appear elsewhere.

\subsection{Fishbone-type mode}

Similar to the JET ELM-free H-mode plasmas [23], the bursting fishbone-type MHD is the most commonly observed magnetic fluctuation in TFTR high power (tangential NBI) supershot plasmas. Due to many differences between the TFTR 
supershot and PDX plasmas on which the fishbone mode was first reported [24], it is not completely clear whether the observed bursting 1/1 MHD mode is the same as the PDX fishbone mode although they have many similarities. Figure 22 shows the electron temperature evolution in the fishbone-type mode phase. It is seen that although the onset of the fishbone bursting mode does not cause a decay of the plasma temperature, it clearly terminates the ramping-up phase of the central $T_{e}$ and seems to correlate with the saturation of the performance or transition to steady-state. Besides a corresponding $10-20 \%$ enhancement in the fusion product loss [Fig. 22(c)], a fishbone burst can cause a $\sim 2-5 \%$ drop in the central $T_{e}$ [Fig. 22(a)] and a very small $(\leqslant 0.2 \%)$ drop in neutron yield. The time evolution of the central electron density does not seem to be affected by the fishbone-type mode as shown in Fig. 22(d). (The time resolution for the $n_{e}$ measurement is $\sim 10$ msec.) In the bursting phase, an $m / n=1 / 1$ type oscillation can be seen in the central $T_{e}$ profile in Fig. 23(a) and (b). A comparison of the electron temperature fluctuations in the bursting and non-bursting phases measured with the ECE diagnostic system is shown in Fig. 23(c). It is interesting to note that the enhancement of the fluctuations during the bursting phase extends over a large region, even out to the $q=2$ surface. This may be due to the high $\beta$ induced toroidal coupling of the $1 / 1$ mode with higher $m$ modes.

In the high power NBI-heated supershot plasmas a few discrete sawtooth crashes are often seen during the beam injection phase, especially when the limiter conditions are not optimum. These sawtooth crashes often happened in the fishbone bursting phase. It is found that these fishbone-induced sawtooth crashes do not significantly alter the plasma performance. This is due primarily to the short period of the sawtooth crash, small plasma volume involved and the already flattened central temperature profile (due to the fishbone oscillations). Like the $m \geq 2$ tearing-type modes, the stability issue for these fishbone-type modes in the supershot plasmas is still an active research topic $[23,25]$.

\section{MODELING THE TRANSPORT EFFECTS DUE TO MAGNETIC ISLANDS}

The observed deterioration of the central plasma profiles after the onset of the magnetic island cannot be simply explained using the usual perturbation theory 
of resistive MHD. The perturbed magnetic energy available for the growth of the tearing mode from the theory is [26]

$$
\delta W=-\left(\pi / m^{2}\right) r_{s}^{3} \tilde{\psi}_{s}^{2} \Delta_{s}^{\prime}
$$

where the subscript $s$ denotes the value taken at the mode rational surface, and $\tilde{\psi}$ is the perturbed helical magnetic flux function. Here, $\Delta^{\prime}$ is the well-known tearing mode stability parameter [27]. Assuming that the coherent mode we observed is a current-driven tearing mode (i.e., $\Delta_{s}^{\prime}>0$ ) and Eq. (2) can still be used in the island saturation phase, one can estimate the scaling between the magnetic energy released from the plasma and the island width, by using the formula $w=4 \sqrt{\tilde{\psi} / \mu_{0} j_{z}}$, where $j_{z}$ is the toroidal current density. That is, the variation of the plasma stored energy implied by Eq. (2) will be proportional to $\delta W / W \propto(w / a)^{4}$. This scaling does not seem to agree with what we have observed experimentally as we will see in the following analysis [Fig. 26(b)]. This is because the perturbation theory does not allow for changes of the plasma profile away from the island, as illustrated in Fig. 24. The real situation is, however, a nearly uniform drop of the profile inside the $r<r_{s}$ region. To explain the experiments, transport during and after the development of the magnetic island must be considered.

\subsection{Analytical model}

An analytical model for the energy confinement deterioration induced by a single magnetic island has been developed by Chang and Callen [28] using a one fluid local transport equation to estimate the island-induced global energy deterioration. A simple derivation given in Appendix A shows that when a bad energy confinement region is introduced in the island region $\left[\chi_{e f f} \rightarrow \infty\right.$ for $\left(r_{s}-w / 2\right)<$ $\left.r<\left(r_{s}+w / 2\right)\right]$, the plasma temperature profile inside the $r<\left(r_{s}-w / 2\right)$ region will exhibit a nearly uniform drop:

$$
\begin{aligned}
\delta T & \equiv T^{0}(r)-T(r) \\
& =-\left(\frac{d T^{0}}{d r}\right)_{s} w+\mathcal{O}\left(w^{2} / a^{2}\right)
\end{aligned}
$$

where $T^{0}(r)(T(r))$ denotes the temperature before (after) the onset of the island. Assuming the plasma density and heating profiles do not change, one can calculate 
the energy deterioration by integrating the product of $n(r) T^{\prime}(r)$ over the plasma volume. The result is

$$
\begin{gathered}
\frac{\delta W}{W}=f\left(r_{s}\right) \frac{w}{a}+\mathcal{O}\left(w^{2} / a^{2}\right) \\
f\left(r_{s}\right)=\frac{20}{3}\left(1-\frac{r_{s}^{2}}{a^{2}}\right)\left[1-\left(1-\frac{r_{s}^{2}}{a^{2}}\right)^{3}\right] \frac{r_{s}}{a}
\end{gathered}
$$

where peaked model profiles have been used [i.e., $n(r)=n(0)\left(1-r^{2} / a^{2}\right)^{2}, T^{0}(r)=$ $\left.T^{0}(0)\left(1-r^{2} / a^{2}\right)^{2}\right]$. The coefficient $f\left(r_{s}\right)$ is plotted in Fig. 25. Equation (5) is an improved formula with respect to the ones obtained in Ref. [28], which were used in the study of the MHD induced confinement deterioration in JET [29]. For most of the supershot plasmas analyzed in Section 2 , the mode rational surface for the $3 / 2$ or $4 / 3$ mode is around $r_{s} / a \simeq 0.4$. Therefore, we have $f\left(r_{s}\right) \sim 1$. This model predicts that the energy deterioration is nearly equal to the ratio of $w / a$. It is worth pointing out that although the assumption of large local transport in an annular ring around the mode rational surface is not consistent with the observed clear island structure discussed in Section 3, it can be shown [28] that this assumption is effectively equivalent to a magnetic island model.

To compare the model with TFTR experiments, we use the following method to estimate the island width. The perturbed magnetic field $\tilde{B}_{r}$ at the mode rational surface is calculated by solving a linear, cylindrical, ideal, low $\beta$ tearing mode equation using the $q$ profile taken from a transport code (SNAP) [30] and scaled to the edge $\tilde{B}_{\theta}$ measured with Mirnov coils. The equation

$$
w_{\text {eff }}=g \sqrt{\left(\frac{r \tilde{B}_{r} q}{m B_{\theta} q^{\prime}}\right)_{s}},
$$

is used to calculate an effective island width $w_{\text {eff }}$. Due to uncertainties on the calculated $\tilde{B}_{r}\left(r_{s}\right), q(r)$ and some possible stochastic region around the islands, a constant $g$ is introduced. It is determined by matching the measured island width, when the island is large and can be identified, to the calculated one. For example, in Fig. 17(a), a $\sim 8-10 \mathrm{~cm}$ flat spot is seen on the $T_{e}$ profile acrossing the island center. The $w_{\text {eff }}$ used in this case is $\sim 9 \mathrm{~cm}$. The comparison between the theoretical model and experimental measurements is shown in Fig. 26(a). To extend the parameter regime, the $2 / 1$ mode data from the $R=2.60 \mathrm{~m}$ plasmas are also included. As we can see, the model predictions agree with the measurements 
reasonably well. In other words, the energy deterioration due to the development of magnetic islands is roughly proportional to the island width of the dominant mode [Fig. 26(b)]. From this model we see that the reason for the weak effect of the $m / n=5 / 4$ modes and some of the $4 / 3$ and $3 / 2$ modes is mainly because their island sizes are so small. This agreement between the analytical model and experimental results seems to indicate that the introduction of a bad confinement region around the magnetic island is the dominant transport process involved in the MHD correlated performance deterioration.

\subsection{Numerical simulation}

A $3 / 2$ or $4 / 3$ MHD mode in TFTR supershots takes typically $\sim 250-450 \mathrm{~ms}$ to saturate (see Figs. 1-3), which is longer than the plasma energy confinement time $\left(\tau_{E} \lesssim 180 \mathrm{~ms}\right)$. Therefore, the process of island evolution and its effect on the global plasma parameters cannot be studied without consideration of the transport processes. To study the dynamical features of the island-induced performance deterioration and to compare them with experimental measurements, numerical simulations have been employed. Two transport models have been used in the following analysis. One is a four-parameter model which consists of four coupled transport equations for the fast ions density $\left(n_{f}\right)$, electron density $\left(n_{e}\right)$, electron temperature $\left(T_{e}\right)$ and thermal ion temperature $\left(T_{i}\right)$. This is a fully predictive model. The other numerical model used is the TRANSP code [31].

\subsubsection{Four-parameter numerical modeling}

In the high power NBI heated supershot plasmas the basic dynamics of the plasma performance are determined by the balance between the beam heating and fueling, and plasma transport (particles and energy). The plasma consists of three main species: electrons, thermal ions and fast beam ions. The following four coupled transport equations are adopted to simulate the evolution of the NBI heated plasmas:

$$
\begin{aligned}
& \frac{\partial n_{f}}{\partial t}=\dot{n}_{f}+\frac{1}{r} \frac{\partial}{\partial r} r D_{f} \frac{\partial n_{f}}{\partial r}-\frac{n_{f}}{\tau_{f}} \\
& \frac{\partial n_{e}}{\partial t}=\dot{n}_{f}^{\text {ionization }}+\frac{1}{r} \frac{\partial}{\partial r} r D_{e} \frac{\partial n_{e}}{\partial r}
\end{aligned}
$$




$$
\begin{aligned}
& \frac{3}{2} n_{e} \frac{\partial T_{e}}{\partial t}=E_{b} \dot{n}_{f} G_{e}+\frac{1}{r} \frac{\partial}{\partial r} r n_{e} \chi_{e} \frac{\partial T_{e}}{\partial r}-Q_{e i} \\
& \frac{3}{2} n_{i} \frac{\partial T_{i}}{\partial t}=E_{b} \dot{n}_{f} G_{i}+\frac{1}{r} \frac{\partial}{\partial r} r n_{i} \chi_{i} \frac{\partial T_{i}}{\partial r}+Q_{e i}
\end{aligned}
$$

where $\dot{n}_{f}$ is the total beam fueling rate (from charge exchange and ionization), $\dot{n}_{f}^{\text {ionization }}$ is the beam fueling rate from ionization only, and $D_{f}(r, t), D_{e}(r, t)$, $\chi_{e}(r, t)$ and $\chi_{i}(r, t)$ are transport coefficients (they are defined by the equations and are assumed to not be functions of other plasma parameters). The fast ion slowing down time $\tau_{f}$ is a complicated function of $T_{e}, T_{i}, n_{i}$ and $E_{b}$, where $E_{b}$ is the beam injection energy. An approximate formula used in the simulation is derived in Appendix B. In Eqs. (10) and (11), $G_{i}$ and $G_{e}$ are the beam heating fractions defined by

$$
\begin{aligned}
G_{i} & \equiv \frac{2}{v_{b}^{2}} \int_{v_{1}}^{v_{b}} \frac{v d v v_{c}^{3}}{v^{3}+v_{c}^{3}}, \\
G_{e} & =\left(1-\frac{3 T_{i}}{2 E_{b}}\right)-G_{i},
\end{aligned}
$$

where $v_{b}=\sqrt{2 E_{b} / m_{b}}, v_{c}=\sqrt{2 E_{c} / m_{b}}$ and $E_{c} \simeq 19 T_{e}(\mathrm{keV})$. The electron-ion energy exchange through collisions is expressed in $Q_{e i}$, which is evaluated using [32]:

$$
Q_{e i}=\frac{3 m_{e} n_{e}}{2 m_{p} \tau_{e}}\left(T_{e}-T_{i}\right)
$$

The main assumptions made in the simulation for simplification are: 1) Profiles of the beam fueling sources $\left(\dot{n}_{f}, \dot{n}_{f}^{\text {ionization }}\right)$ are given and do not change in time. This assumption can be reasonably justified by TRANSP calculations. The profiles of the beam fueling rates at different times during the NBI phase calculated by the TRANSP code for discharge 66869 are shown in Fig. 27. This is the discharge which has a $3 / 2$ mode described in Fig. 1. The calculation shows that within the analysis uncertainty the beam fueling rates do not change during the NBI phase. 2) The impurity density is neglected. Therefore the thermal ion density can be determined from quasineutrality:

$$
n_{i} \simeq n_{e}-n_{f} .
$$

3) The transport coefficient profiles arc modeled to obtain a supershot-like plasma and assumed not changing with time. 4) When the "island" is turned on, a locally 
enhanced transport region is introduced instantaneously around the mode rational surface.

The global neutron emission rate is calculated assuming

$$
\begin{aligned}
S_{n} & =\sum_{j, k} \int d V n_{j} n_{k}\langle\sigma v\rangle_{j k} \\
& =S_{n}^{b-b}+S_{n}^{b-t}+S_{n}^{t-t}
\end{aligned}
$$

where $S_{n}^{b-b}=(1 / 2) \int d V n_{f}^{2}\langle\sigma v\rangle_{b b}$ is the beam-beam neutron yield, $S_{n}^{b-t}=\int d V n_{f} n_{i}\langle\sigma v\rangle_{b t}$ is the beam-target neutron yield, and $S_{n}^{t-t}=(1 / 2) \int d V n_{i}^{2}\langle\sigma v\rangle_{t-t}$ is the target: target or thermonuclear neutron yield. We use the empirical formula from the $N R L$ formulary [32] to calculate the thermal reaction rate and use an empirical table [33] to estimate the beam-target part. The beam-beam neutron yield is then determined by specifying the ratio $\left(\left.S_{n}^{b-b}\right|_{\max }\right) /\left(\left.S_{n}^{b-t}\right|_{\max }\right)$. The total stored energy is given by the sum of the three plasma components:

$$
W_{t o t}=W_{e}+W_{i}+W_{b}
$$

where $W_{j}=(3 / 2) \int d V n_{j} T_{j}, j=e, i, b$.

Typical simulation results are shown in Figs. 28-30. The main parameters used in the simulation are: $E_{b}=60 \mathrm{keV}$ (assuming that all the injected beam ions have the same energy), $r_{s} / a=0.42, w / a=0.18,\left.S_{n}^{b-b}\right|_{\max }=\left.0.7 S_{n}^{b-t}\right|_{\text {max }}$. A factor of 4 enhancement in the island region is used for the transport coefficients $\chi_{e}, \chi_{i}$ and $D_{e}$. [Small and constant $D_{f}\left(D_{f} / D_{e}(0)<1 / 2\right)$ has been assumed.] The energy confinement time $\tau_{E}\left[\equiv W /\left(P_{B}-d W / d t\right), P_{B}=30 \mathrm{MW}\right.$ used $]$ turns over first at $\sim 100 \mathrm{~ms}$ after the start of "NBI", which simply reflects the change of the energy growth rate $(d W / d t)$. In the simulation without the island, the plasma performance (Fig. 29) saturates at $\sim 0.4 \mathrm{sec}$. Then, a small roll-over $(\leqslant 10 \%$ ) is seen on the neutron yield and stored energy ( $\leqslant 5 \%)$. The neutron yield rolls earlier than the stored energy (which agrees with supershot observations). The reason for this "natural" roll-over is that, due to the high central beam fueling rate and small particle transport $\left(D_{e}(0) \simeq 0.2 \chi_{e}(0)\right.$ has been used to obtain a supershot-like density evolution), the plasma density quickly increases after the start of NBI. This leads to a decrease of the fast ion slowing down time in the center. Then, the central fast ion density starts to decrease at $\sim 200-300 \mathrm{~ms}$ 
after the start of NBI, which results in a drop in the beam-beam neutron yield [Fig. 29(a)].

When the island model is introduced, a 20\% deterioration in total neutron yield and $a \sim 12 \%$ deterioration in stored energy are obtained. The evolution of the plasma profiles after the development of the island is shown in Fig. 30. It is seen that the time for the island transport effect to "propagate" to the center is about $10-20 \mathrm{~ms}$, while the full "diffusive" transport decrease in central parameters takes about few hundred miliseconds. Despite the simplification made in the model on the instantancous onset of the island, the time scales we obtained here are roughly consistent with experimental obscrvations, see Fig. 18. The calculated drop of $T_{i}$ and $T_{e}$ across the $r<\left(r_{s}-w / 2\right)$ region is not quite uniform due to the involvement of the particle transport (as can be expected, see the discussion in Appendix A). This detail does not seem to agree well with the experimental observations (see Figs. 17 and 19). Nevertheless, the simulation shows that despite the many simplifications made in the model, the basic dynamical features are consistent with experimental results.

\subsubsection{TRANSP modeling}

A numerical simulation of the island effect using a more sophisticated transport analysis code TRANSP [31] has also been explored. Similar to the previous analysis, we assume that the existence of the island is equivalent to a bad confinement region around the mode rational surface. In the simulation, $T_{e}$ and $T_{i}$ are calculated by specifying the effective $\chi_{e}(r, t)$ and $\chi_{i}(r, t)$ [using the $\chi_{e}(r, t)$ from a TRANSP run for a non-deteriorated discharge (66868) and assuming $\left.\chi_{i}(r, t)=2 \chi_{e}(r, t)\right]$. At present, the TRANSP code does not have the option for calculating the $n_{e}$ from a given $D_{e}$ profile. Therefore, the measured density (without deterioration) is used in the simulation. The island width used is $w / a \simeq 0.15$ at $r_{s} / a \simeq 0.4$. The diffusion coefficient enhancement factor in the island region is $\sim 20$. Fig. 31 shows the neutron yield evolution from two simulations - with and without an island present. The island model is turned on for $300 \mathrm{msec}$ (from $3.55 \mathrm{sec}$ to $3.85 \mathrm{sec}$ ). A $\sim 25 \%$ deterioration on the total neutron yield is seen during this period. This deterioration is mainly in the beam-target and thermal components. (No anomalous fast ion transport was assumed in the model.) The time evolution of $T_{e}$ and $T_{i}$ 
profiles after the onset of the island is shown in Fig. 32. The calculated $T_{e}$ profile evolution after the onset of the island is very similar to the measurement shown in Fig. 17. The larger drop in the central $T_{i}$ than the $T_{e}$ is expected from the theory $\left[\mathrm{Eq}_{\mathrm{q}}\right.$ (4)], since the ion temperature gradient around the mode rational surface is higher. Note that due to $\chi_{i}^{e f f}(r, t)>\chi_{e}^{e f f}(r, t)$ and $\left|\nabla T_{i}\right|>\left|\nabla T_{e}\right|$ in the supershot core region, the propagation of the island effect to the plasma center is faster for the $T_{i}(<20 \mathrm{mscc})$ than for the $T_{e}(\leqslant 50 \mathrm{msec})$. Also, note that the decrement of both $T_{e}$ and $T_{i}$ are nearly constant across the $r<r_{s}$ region, which also agrees with the analytical model Eq. (4).

\section{DISCUSSION OF THE EFFECTS OF RECYCLING ON THE PER- FORMANCE DETERIORATION}

It is well-known that a strong inward impurity flux (e.g., Carbon blooms) can destroy the normal plasma performance [6]. Through intensive wall conditioning before a supershot experiment and accurate alignment of the limiter tiles, the bloom event has been suppressed in normal TFTR operation. However, a milder increase in the recycling rate (inferred from the edge $C_{I I}$ and $H_{\alpha}$ emissivity measurements) is often observed in TFTR NBJ heated supershots, especially in larger major radius plasmas. Correlations between high maximum performance and low edge recycling rate has also been observed [34].

Figure 33 shows three sequential discharges from a major radius scan experiment. The $C_{I I}$ and $H_{\alpha}$ data are normalized to their pre-beam values. No coherent MHD modes are observed in these three discharges, except for the fishbone type modes in the two smaller major radius plasmas. As can be seen, the neutron yield deterioration increases as the plasma major radius is increased from $R=2.45 \mathrm{~m}$ to $R=2.59 \mathrm{~m}$. The $C_{l l}$ and $H_{\alpha}$ emissivities are continuously increasing, corresponding to the larger deterioration in the plasmas with larger major radius.

To describe this phenomenon, a "recycling parameter" is introduced:

$$
K \equiv\left\langle\frac{\delta C_{I I}}{C_{I I}\left(t_{0}\right)}\right\rangle_{t}+\left\langle\frac{\delta H_{\alpha}}{H_{\alpha}\left(t_{0}\right)}\right\rangle_{t}
$$

where $\delta C_{I I} \equiv C_{I I}(t)-C_{I I}\left(t_{0}\right)$, and \langle\rangle$_{t}$ denotes the time average of the argument. In the following analysis, $t_{0}$ is chosen to be $\sim 3.4 \mathrm{sec}$ which is about $50 \mathrm{~ms}$ before the peak neutron yield time for most discharges. The time window for the averag- 
ing is from $3.4 \mathrm{sec}$ to $3.9 \mathrm{sec}$. (The beam duration is from 3 to $4 \mathrm{sec}$.) Therefore, the parameter $K$ is a measure of the time averaged, relative change in the sum of Carbon and $H_{\alpha}$ emissivities. Generally speaking, in the non-deteriorated discharges, such as the discharges shown in Fig. 1, the $\delta C_{11} / C_{11}$ term in Eq.(18) is negative and $\delta I_{\alpha} / H_{\alpha}$ term is positive. They tend to cancel each other and give $K \sim 0$. In larger radius plasmas, both terms become positive and make $K>0$, as can be seen from Fig. 33.

Figure 34 plots the data from the major radius scan experiment. It is clearly seen that the recycling parameter $K$ tends to increase as the major radius increases. Also, a good correlation can be seen between the parameter $K$ and the deterioration in the neutron yield. A larger database for the $R=2.60 \mathrm{~m}$ plasmas is plotted in Fig. 35. The dominant MIID mode involved in these discharges is also shown in the graph. For large MIID) activity cases $(m / n=2 / 1$ and most $3 / 2$ modes), the correlation between $\delta S_{n} / S_{n}$ and $K$ is poor, which secms to indicate that in these cases the MHD is the major mechanism for the performance deterioration. On the other hand, when the MHD is weak, a good correlation is observed between the drop in the neutron yield and the increase in the recycling. In the case when we have both MIID and large $K$, it is not clear which mechanism dominates the deterioration process. The correlation between the increase of the recycling rate and the performance decay has also been observed in the NBI plus ICRF (ion cyclotron range frequency) heating experiments where a large radiation rise often follows the injection of the ICRF power during the NBI phase [35].

The preceding paragraph gave a phenomenological description of the possible correlation between the variation of recyeling rate and performance deterioration. It is not clear how the edge recycling rate affects the plasma performance. A few possibilities are as follows. The increases in $I$ correspond to: (a) Increasing the plasma coherent MHD activity. One extreme example is the minor disruption induced low $(m, n)$ MHD, which is often observed in supershots; (b) Increasing the edge density and therefore, decreasing the beam penetration. It has been observed [36] that the maximum supershot performance (neutron yicld, for example) closely correlates with the beam fueling profile, parameterized by a ratio of electron density peakedness and the line integrated density. (c) Increasing the plasma anomalous transport due to changes of the impurity content. A more detailed study of the 
recycling effects is beyond the scope of this paper.

\section{CONCLUSIONS}

A strong correlation between the development of low $(m, n)$ MHD and performance deterioration has been observed in TFTR supershots. Detailed shot-to-shot comparison and statistical data analysis using the $1992 \mathrm{TFTR}$ database shows that in the high performance supershot regime with $P_{B}>10 \mathrm{MW}$ and $\tau_{E} / \tau_{E}^{L}>1.5$, the probability of having the $m / n=3 / 2$ and $4 / 3$ mode MIID is about $30 \%$ in $R=2.45$ $\mathrm{m}$ supershots. The MHD correlated performance deterioration depends upon the amplitude of the mode (or the island width). A deterioration up to $\sim 30 \%$ in total D.D fusion neutron yield and $\sim 15 \%$ on the stored energy has been observed when the island width is about $w / a \simeq 0.12$ (or $w \sim 9-10 \mathrm{~cm}$ ). A stronger deterioration has also been observed for the $m / n=2 / 1$ mode case, in $R \simeq 2.60 \mathrm{~m}$ plasmas, where $\mathrm{a} \sim 60 \%$ drop in neutron yicld and $\sim 30 \%$ drop in stored energy correlated with the development of a $w / a \sim 0.30$ island. The distinct feature of the island induced plasma profile evolution is that, following the slow growth of the mode amplitude, the profile $\left(n_{e}, T_{e}\right.$ and $\left.T_{i}\right)$ inside the $r<r_{s}$ region decays almost uniformly; while the profile outside the $r>r_{s}$ region remains relatively unchanged. Magnetic island-type structures have been observed around the mode rational surfaces for strong $2 / 1,3 / 2$ and $4 / 3$ modes. The $m / n=1 / 1$ fishbone-type mode, which is the most often observed MHD in the high quality supershots, does not usually cause a decay of the plasma parameters in $R=2.45 \mathrm{~m}$ plasmas. However, it terminates the rising phase of central $T_{e}$ and secms to correlate with the saturation of the plasma performance of the supershots. Strong MHD-induced fusion product losses have also been observed. These results show that the low $(m, n)$ coherent MHD can have a strong impact on the plasma performance when the other destructive events (like major or minor disruptions, blooms, etc.) are avoided.

The island-induced global energy deterioration has been modeled assuming an enhanced transport region around the mode rational surface without invoking (a) enhancements in local transport elsewhere or (b) transport of beam ions by MHD. Good agreement with the experimental measurements has been observed using both an analytical model and numerical simulations.

In supershots with larger plasma major radius $(R \simeq 2.52 \mathrm{~m}$ and $R \simeq 2.60 \mathrm{~m}$ ) 
the probability of the coherent MHD events becomes much smaller. The non-MHD correlated performance deterioration in the larger size plasmas correlates with a continuous increase of the recycling rate when the plasma reaches its maximum performance, but the corresponding physical mechanism is not clear. 


\section{ACKNOWLEDGMENTS}

The authors would like to thank Dr. R. Hawryluk, Dr. D. Meade, Dr. R. Davidson and the whole TFTR group for their encouragement and support of this research. We would also like to acknowledge the help from Dr. D. Mikklelsen on understanding and utilizing neutron yield empirical tables. This research was supported by US DoE Contract No. DE-FG02-92ER54139 and DE-AC02-76-CHO3073. 


\section{APPENDIX A:}

In a cylindrical one-fluid plasma, the equilibrium energy balance equation $Q=$ $\nabla \cdot \mathrm{q}=-\nabla \cdot n \chi \nabla T$, can be written as:

$$
-\frac{d}{r d r} r n \chi \frac{d T}{d r}=Q(r)
$$

where $Q$ is the total heating power density, $\chi$ is the effective heat diffusivity including energy convection processes. $\Lambda$ ssuming that the profiles of $n(r), \chi(r)$ and $Q(r)$ are given, we can integrate Eq. (A.1) to obtain

$$
\frac{d T}{d r}=-\frac{1}{r n \chi} \int_{0}^{r} Q r d r
$$

Integrating this equation once more and assuming zero edge temperature, we have

$$
T^{\prime}(r)=\int_{r}^{c} \frac{d r}{r n \chi} \int_{0}^{r} Q r d r .
$$

This result indicates that when a large heat transport region is introduced locally around the mode rational surface $r_{s}$ with width $w$, the temperature profile outside the "island" $r>\left(r_{s}+w / 2\right)$ will not be affected. The temperature profile inside the mode rational surface can be written as

$$
\begin{aligned}
T(r) & =\left(\int_{r}^{r_{s}-w / 2}+\int_{r_{s}+w / 2}^{a}\right) \frac{d r}{r n \chi} \int_{0}^{r} Q r d r \\
& =\left(\int_{r}^{a}-\int_{r_{s}-w / 2}^{r_{s}+w / 2}\right) \frac{d r}{r n \chi} \int_{0}^{r} Q r d r \\
& =T^{0}(r)-w\left(\frac{1}{r n \chi}\right) \int_{s}^{r_{s}} Q r d r+\mathcal{O}\left(w^{2} / a^{2}\right),
\end{aligned}
$$

where $T^{0}(r)$ is the temperature profile before introducing the island. By using Eq. (A.2), one obtains the difference in the temperature profile induced by the island,

$$
\begin{aligned}
\delta T^{\prime} & \equiv T^{0}(r)-T(r) \\
& =-\left(\frac{d T^{0}}{d r}\right)_{s} w+\mathcal{O}\left(w^{2} / a^{2}\right), \quad \text { for } r<\left(r_{s}-w / 2\right) .
\end{aligned}
$$

This result shows that to the first order in $w / a \ll 1$, the drop of the core temperature inside the island is uniform. It only depends on the local temperature gradient at the mode rational surface. 
In this calculation the magnetic island effect on particle transport has been neglected. Physically, the magnetic reconnection should also effectively enhance the particle transport just as it does the heat transport discussed above. If one assumes that the $\chi$ and $D$ do not depend on plasma density or temperature, one can prove using the same approach described above that consideration of the particle transport will make the temperature drop [Eq. (A.5)] nonuniform, i.e., $\delta T(r \sim 0)<\delta T\left(r \lesssim r_{s}\right)$. This effect can be seen in the numerical simulation (see Fig. 30). 


\section{APPENDIX B: Derivation of the fast ion slowing down time}

Using the formula provided in Ref. [32], we can calculate the energy loss of the fast beam ions to the background elections and ions:

$$
\frac{d E_{f}}{d t}=\left.\frac{d E_{f}}{d t}\right|_{i}+\left.\frac{d E_{f}}{d t}\right|_{e} .
$$

Here,

$$
\begin{aligned}
\left.\frac{d E_{f}}{d t}\right|_{i} & =-\nu_{E}^{f / i} E_{f} \\
& =-2\left[\frac{m_{f}}{m_{i}} \psi\left(x^{f / i}\right)-\psi^{\prime}\left(x^{f / i}\right)\right]\left(\frac{E_{c}}{E_{f}}\right)^{3 / 2} \frac{n_{i}}{n_{e}} \frac{1}{\tau_{s e}} E_{f}
\end{aligned}
$$

and

$$
\left.\frac{d E_{f}}{d t}\right|_{e} \simeq-\frac{2 E_{f}}{\tau_{s e}} \quad \text { for } \quad x^{f / e} \ll 1,
$$

where $x^{\alpha / \beta} \equiv E_{\alpha} / E_{\beta}, \psi(x)=(2 / \sqrt{\pi}) \int_{0}^{x} d t \sqrt{t} e^{-t}, E_{c} \simeq 19 T_{e}(k e V)$ is the critical energy and $\tau_{s e}$ is the fast ion slowing down time for collisions with electrons

$$
\tau_{s e}=\frac{3 m_{e} m_{f} v_{e}^{3}}{16 \sqrt{\pi} n_{e} e^{4} \ln \Lambda} \propto \frac{T_{e}^{3 / 2}}{n_{e}} .
$$

Substituting Eqs. (B.2) and (B.3) into Eq. (B.1), we have (taking $m_{f}=m_{i}$ )

$$
\frac{d E_{f}}{2 E_{f}\left[1+\left(\psi-\psi^{\prime}\right)\left(n_{i} / n_{e}\right)\left(E_{c} / E_{f}\right)^{3 / 2}\right]}=-\frac{d t}{\tau_{s e}} .
$$

We assume that a fast beam ion is considered thermalized when its energy $E_{f}$ equals to $3 T_{i} / 2$. Integrating Eq. (B.5), we obtain the fast beam ion slowing down time:

$$
\tau_{f} \simeq \tau_{s e} \int_{3 T_{i} / 2}^{E_{b}} \frac{d E_{f}}{2 E_{f}}\left[1+\left(\psi\left(x^{f / i}\right)-\psi^{\prime}\left(x^{f / i}\right)\right) \frac{n_{i}}{n_{e}}\left(\frac{E_{c}}{E_{f}}\right)^{3 / 2}\right]^{-1} .
$$

Here, $E_{b}$ is the beam injection energy. When the ion temperature is low compared to $E_{b}$, i.e., $E_{b} / T_{i} \gg 1, \mathrm{Eq}$. (B.6) reduces to the familiar formula [37]

$$
\tau_{f}=\frac{\tau_{s e}}{3} \ln \left[1+\left(E_{b} / E_{c}\right)^{3 / 2}\right]
$$




\section{References}

[1] STRACHAN, J. D., et al., Phys. Rev. Lett. 58 (1987) 1004.

[2] GOLDSTON, R.J., Plasma Phys. 26 (1984) 87.

[3] THOMPSON, E., et al., Phys. Fluids B 5 (1993) 2468.

[4] STRAIT, E. J., et al., in Controlled Fusion and Plasma Physics (Proc. 20th Eur. Conf. Lisbon, 1993), Vol. 17C, Part I, European Physical Society (1993) 211.

[5] HOSOGANE, N., Phys. Fluids B 5 (1993) 2412. NEYATANI, Y., et al., in Controlled Fusion and Plasma Physics (Proc. 20th Eur. Conf. Lisbon, 1993), Vol. 17C, Part I, European Physical Society (1993) 215.

[6] RAMSEY, A., et al., Nucl. Fusion 31 (1991) 1811.

[7] McGUIRE, K., et al., in Plasma Physics and Controlled Nuclear Fusion Research (Proc. 11 $1^{\text {th }}$ Int. Conf. Kyoto, 1986), Vol.1, IAEA, Vienna (1987) 421. FREDRICKSON, E. D., et al., Rev. Sci. Instrum. 59 (1988) 1797.

[8] BUDNY, R. V., et al., in Controlled Fusion and Plasma Physics (Proc. 20th Eur. Conf. Lisbon, 1993), Vol. 17C, Part I, European Physical Society (1993) 167.

[9] CHANG, Z., et al., in Controlled Fusion and Plasma Physics (Proc. 20th Eur. Conf. Lisbon, 1993), Vol. 17C, Part I, European Physical Society (1993) 207.

[10] FREDRICKSON, E., et al., Rev. Sci. Instrum. 57 (1986) 2084.

[11] CAVAllo, A., et al., Rev. Sci. Instrum. 59 (1988) 889.

[12] MANSFIELD, D. K., et al., Appl. Opt. 26 (1987) 4469.

[13] STAUFFER, F. J., et al., Rev. Sci. Instrum. 59 (1988) 2139.

[14] STRATTON, B. C., et al.,Visible charge exchange recombination spectroscopy on TFTR (invited), in Proceedings of the IAEA Technical Committee Meeting on Time Resolved Two- and Three-Dimensional Plasma Diagnostics, (Nagoya, Japan, November 1990), pp. 78-87, Vienna, 1990, IAEA. 
[15] BELL, M. G., et al., in Plasma Physics and Controlled Nuclear Fusion Research 1988 (Proc. $12^{\text {th }}$ Int. Conf. Nice, 1988), Vol. 1 IAEA, (1989) 27.

[16] TROYON, F., Plasma Phys. Controlled Fusion 26 (1984) 209.

[17] ZWEBEN, S. J., et al., in Plasma Physics and Controlled Nuclear Fusion Research, 1992, paper IAEA-CN-56/A-6-3. ZWEBEN, S.J., et al., Nucl. Fusion 33 (1993) 705.

[18] GREK, B., et al., Observation of large scale structures on the electron temperature profiles measured by thomson scattering, to be published in Nucl. Fusion .

[19] PARK, W., et al., in Plasma Physics and Controlled Nuclear Fusion Research 1990 (Proc. $13^{\text {th }}$ Int. Conf. Washington, D.C., 1990), Vol. 1 IAEA, (1991) 121.

[20] HENDER, T. C., et al., Nucl. Fusion 27 (1987) 1389.

[21] CAlLen, J. D., et al., in Plasma Physics and Controlled Nuclear Fusion Research 1987 (Proc. 11 th Int. Conf. Kyoto, 1986), Vol. 2 IAEA, (1987) 157. CARrerA, R., et al., Phys. Fluids 29 (1986) 889.

[22] MANICKAM, J., et al., Nucl. Fusion 27 (1987) 1461.

[23] NAVE, M. F. F., et al., Nucl. Fusion 31 (1991) 697.

[24] McGuire, K., et al., Phys. Rev. Lett. 50 (1983) 891.

[25] KAITA, R., et al., Phys. Fluids B 2 (1990) 1584.

[26] White, R.B., Rev, Mod. Phys. 58 (1986) 183. VAN DAM, J.W., Editor, From Particles to Plasmas (Addison-Wesley Publishing Company, Inc., 1989) p. 249.

[27] FURTH, H.P., et al., Phys. Fluids 6 (1963) 459.

[28] Chang, Z., CALLEN, J.D., Nucl. Fusion 30 (1990) 219.

[29] SNIPES, J.A., et al., Nucl. Fusion 30 (1990) 205. 
[30] TOWNER, H. H., et al., Rev. Sci. Instrum. 63 (1992) 4753.

[31] HAWRYLUK, R. J., An Empirical Approach to Tokamak Transport, in Physics of Plasma Close to Thermonuclear Conditions, Brussels, 1980, CEC Vol. 1, pp. 19-46. GOLDSTON, R. J., et al., J. Comput. Phys. 43, (1981) 61.

[32] BOOK, D. L., NRL Plasma Formulary, Naval Research Laboratory, NRL Publication 177-4405, Revised ed. (1990).

[33] MIKKELSEN, D., private communication.

[34] STRACHAN, J. D., Studies of global energy confinement in TFTR supershots,Rep. PPPL-2933, Princeton Plasma Physics Lab. 1993.

[35] TAYLOR, G., et al, in Controlled Fusion and Plasma Physics (Proc. 20th Eur. Conf. Lisbon, 1993), Vol. 17C, Part III, European Physical Society (1993) 973. (Also submitted to Plasma Phys. and Controlled Fusion.)

[36] PARK, H., et al., Role of Neutral-Beam Fueling Profile in Energy Confinement and Neutron Emission on TFTR, submitted to Nucl. Fusion.

[37] For example, CALLEN, J. D., et al., in Plasma Physics and Controlled Nuclear Fusic nesearch 1974 (Pro. $13^{\text {th }}$ Int. Conf. Tokyo, 1974) Vol. 1, IAEA, Vienna (1975) 645. 


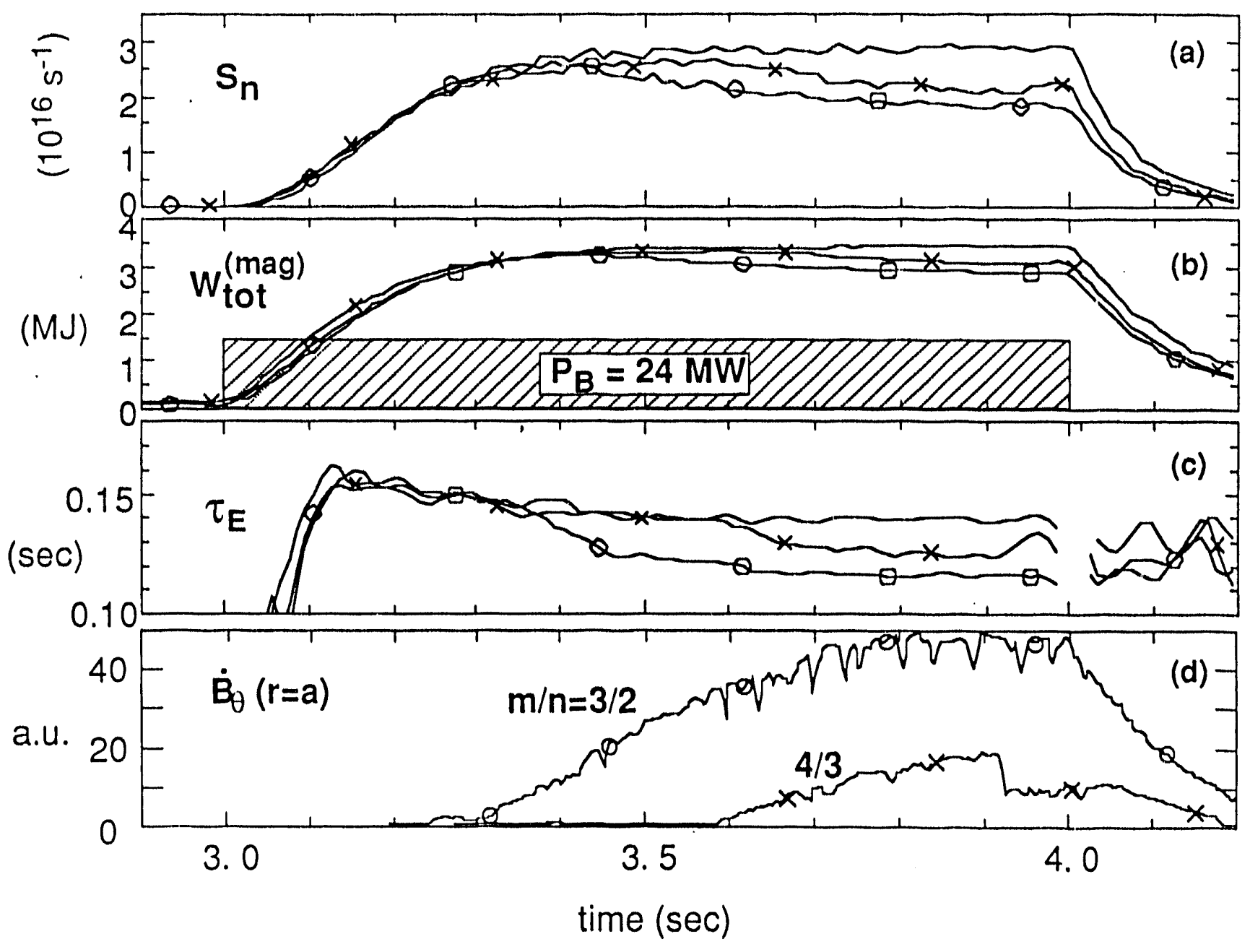

Figure 1: Comparison of (a) total neutron yield $S_{n}$, (b) total stored energy $W_{\text {tot }}$ (measured from a magnetic system) and (c) energy confinement time $\tau_{E}$, for three supershots with similar target plasma and known setup conditions. Here, $\tau_{E} \equiv$ $W_{\text {tot }} /\left(P_{\text {in }}-d W_{\text {tot }} / d t\right), P_{\text {in }}$ is the total input power. The $\tau_{E}$ values before the neutral beam and around the beam turning on and off phases are suppressed. (d) The coherent MHD modes developed in the two discharges exhibiting a performance deterioration. A fishbone-type bursting MHD mode [not shown in (d)] starting at $\sim 3.35 \mathrm{sec}$ (see Fig. 19) is seen in the discharge without performance deterioration (the solid curves without symbols). 


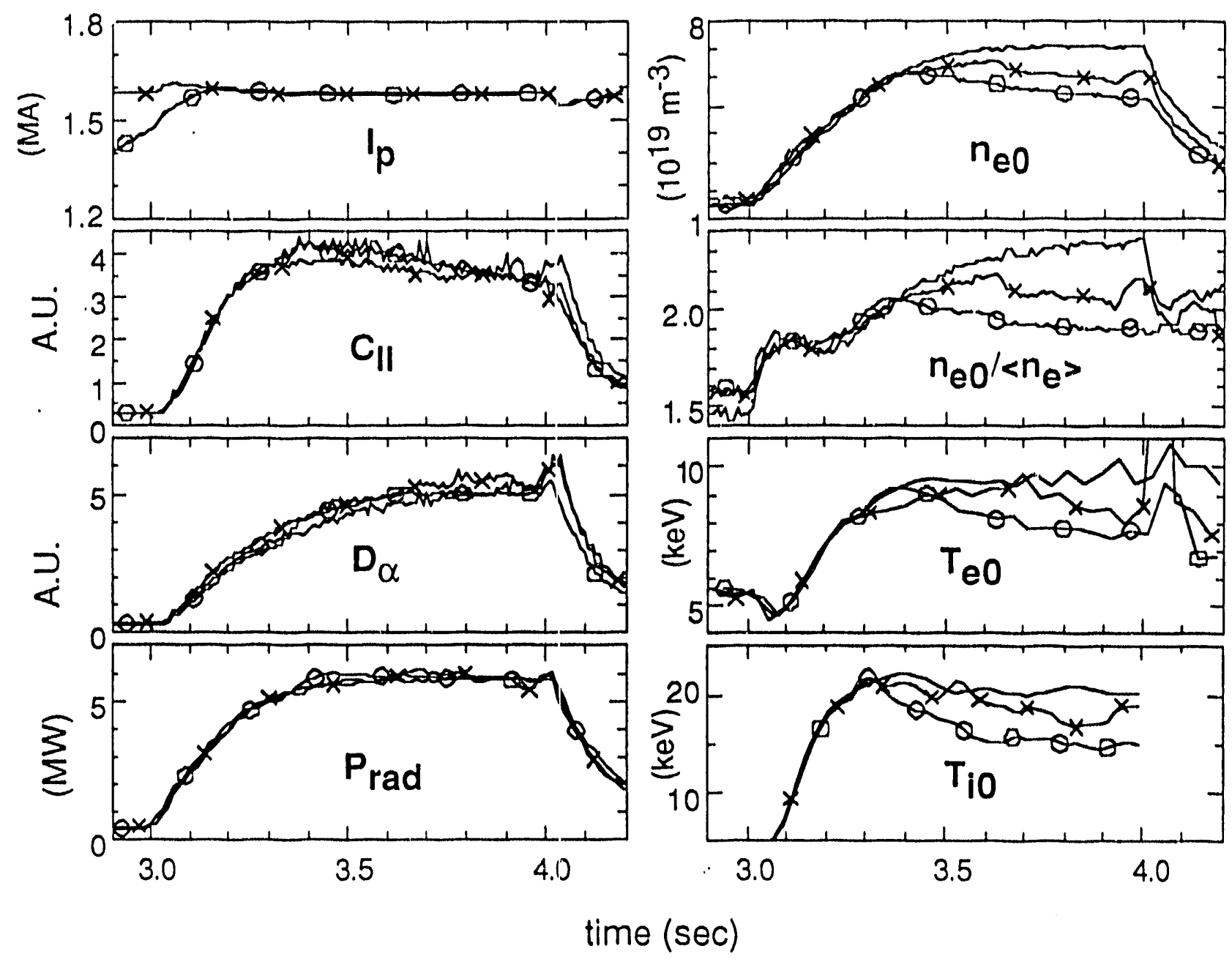

Figure 2: More comparisons of the three discharges shown in Fig. 1. The bottom three figures on the left iilustrate the similarity of the plasma wall conditioning in the NBI phase. The figures on the right display the differences in the performance correlated with different MHD activities. 


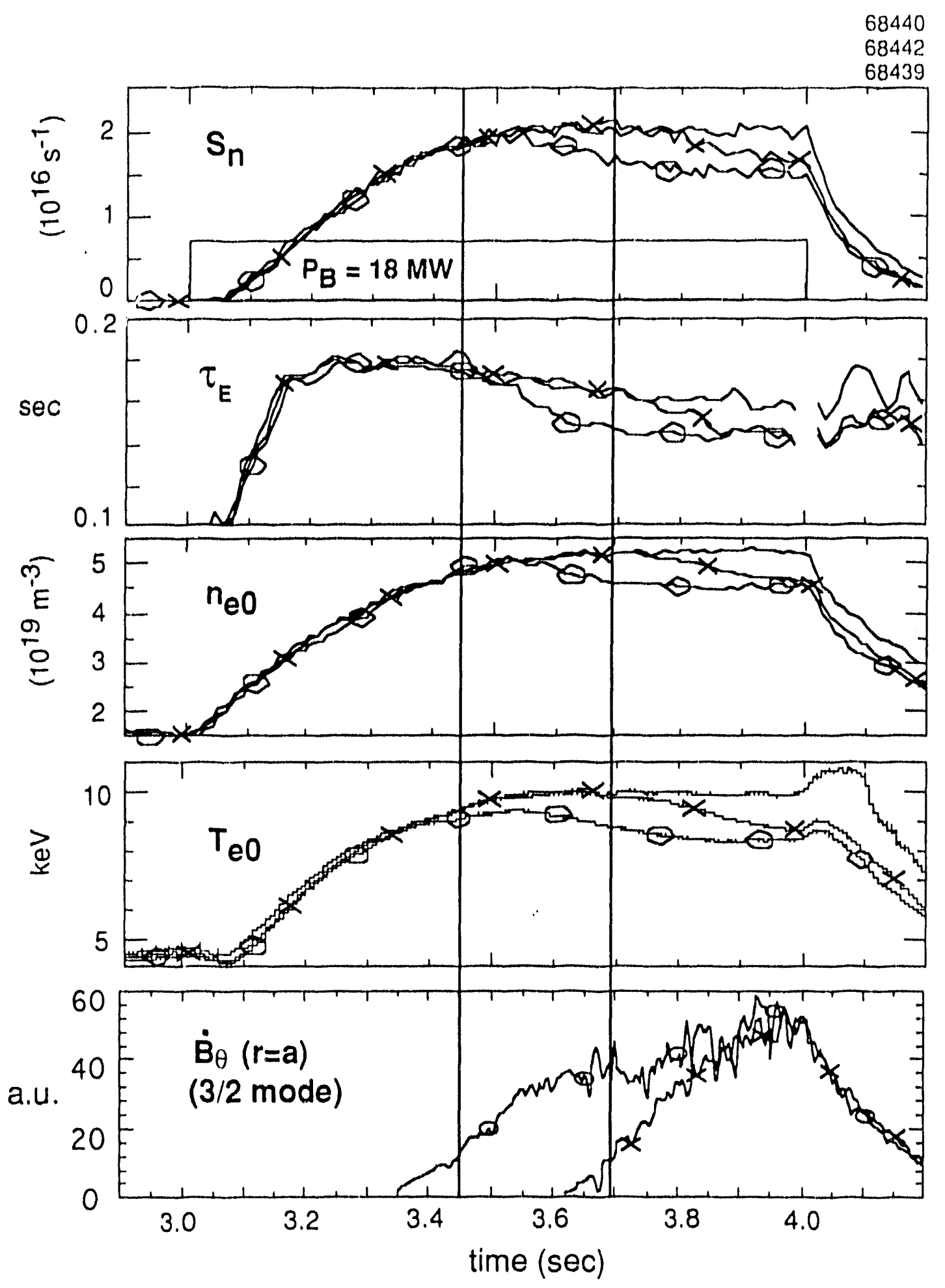

Figure 3: Time correlation between the development of the $m / n=3 / 2$ mode and the performance deterioration. Note that when the mode starts later, the deterioration also happens later. A similar discharge without the $3 / 2$ mode is also shown as a reference. Again, the wall conditioning is almost identical for these discharges. 


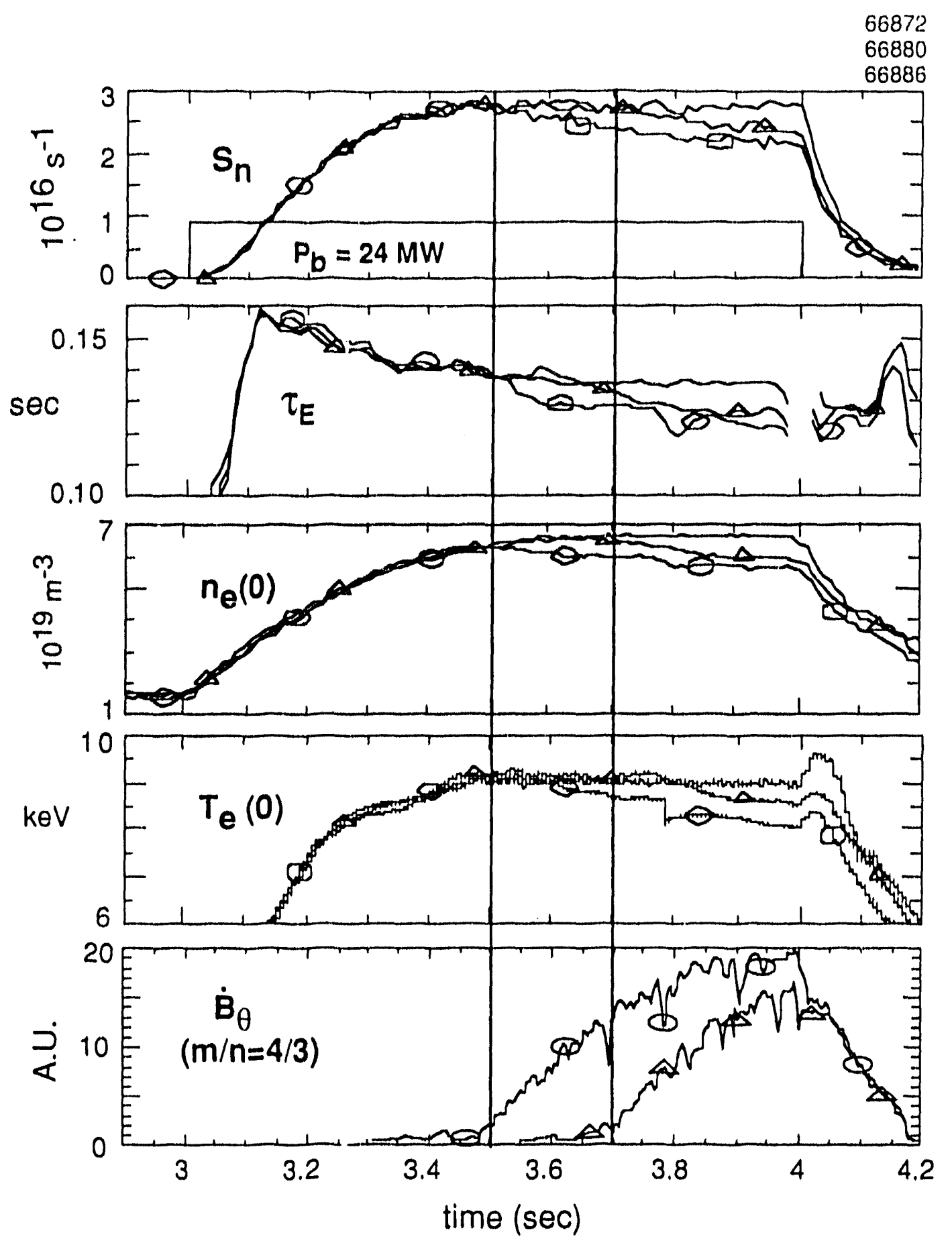

Figure 4: Similar shot-to-shot comparisons as Fig. 3. A close time correlation can be seen between the appearance of the $m / n=4 / 3$ mode and the performance deterioration. 

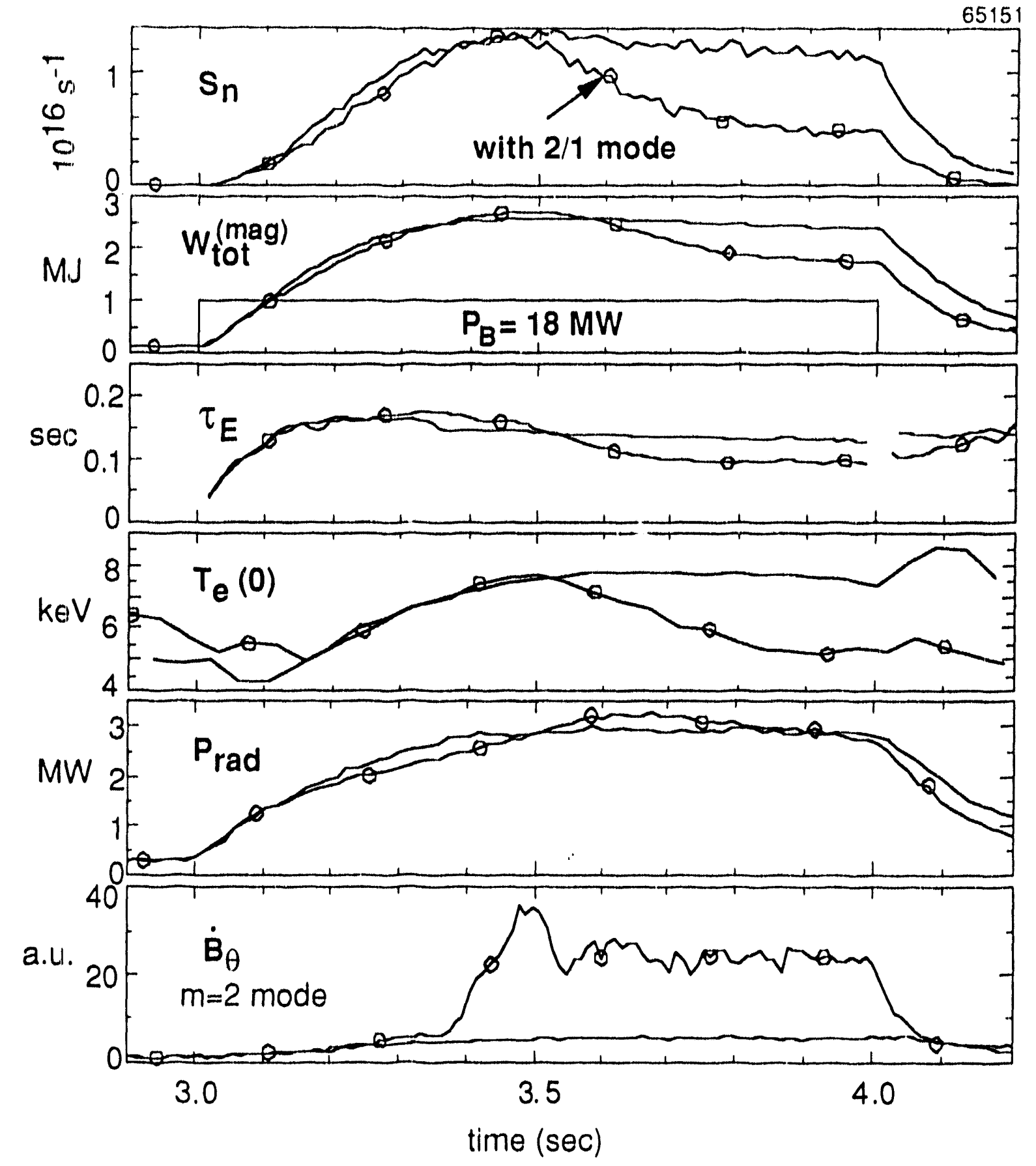

Figure 5: Comparison of two discharges with major radius $R \simeq 2.60 \mathrm{~m}$. A large deterioration is seen in the discharge having a strong $m / n=2 / 1$ mode. 


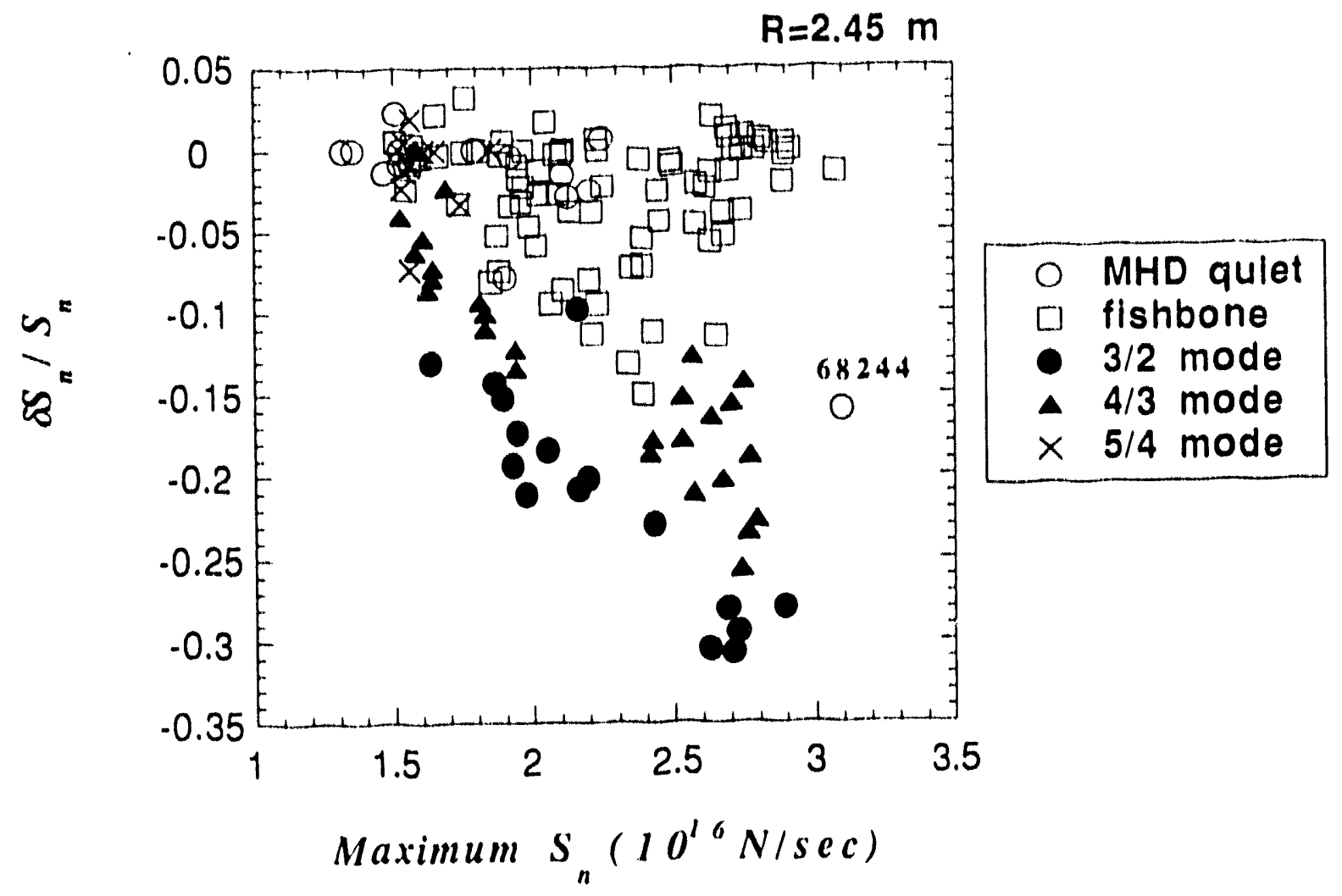

Figure 6: Statistical data analysis of 148 supershot discharges with $R=2.45 \mathrm{~m}$. The neutron yield deterioration parameter $\delta S_{n} / S_{n}$ is defined in Eq. (1). The different symbols denote different dominant MHD modes detected from the Mirnov and ECE diagnostics at around the peak neutron yield time. 


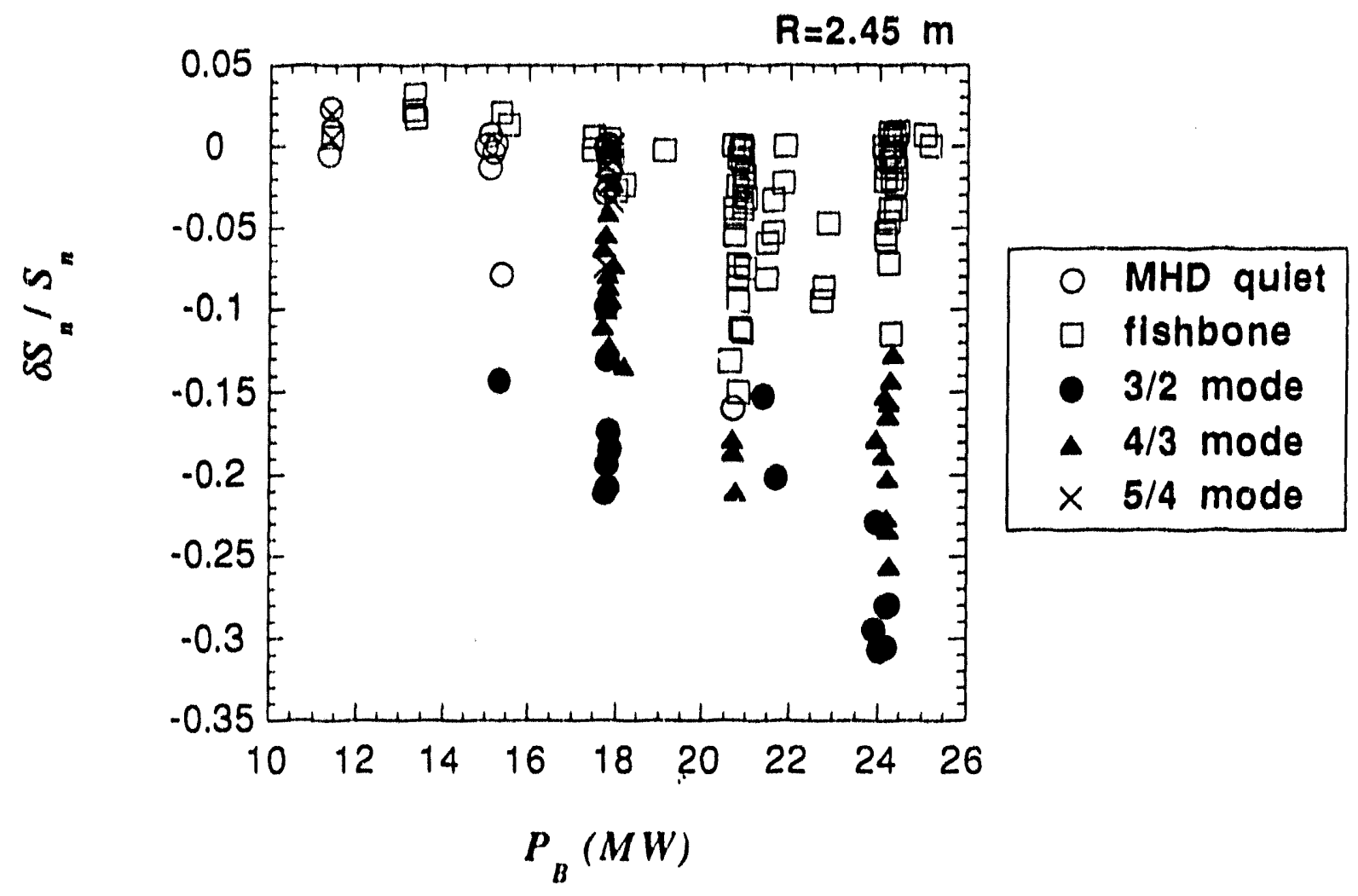

Figure 7: The neutron yield deterioration $\delta S_{n} / S_{n}$ versus the neutral beam power. Note that there is no systematic dependence of the MHD activity on the neutral beam power. 


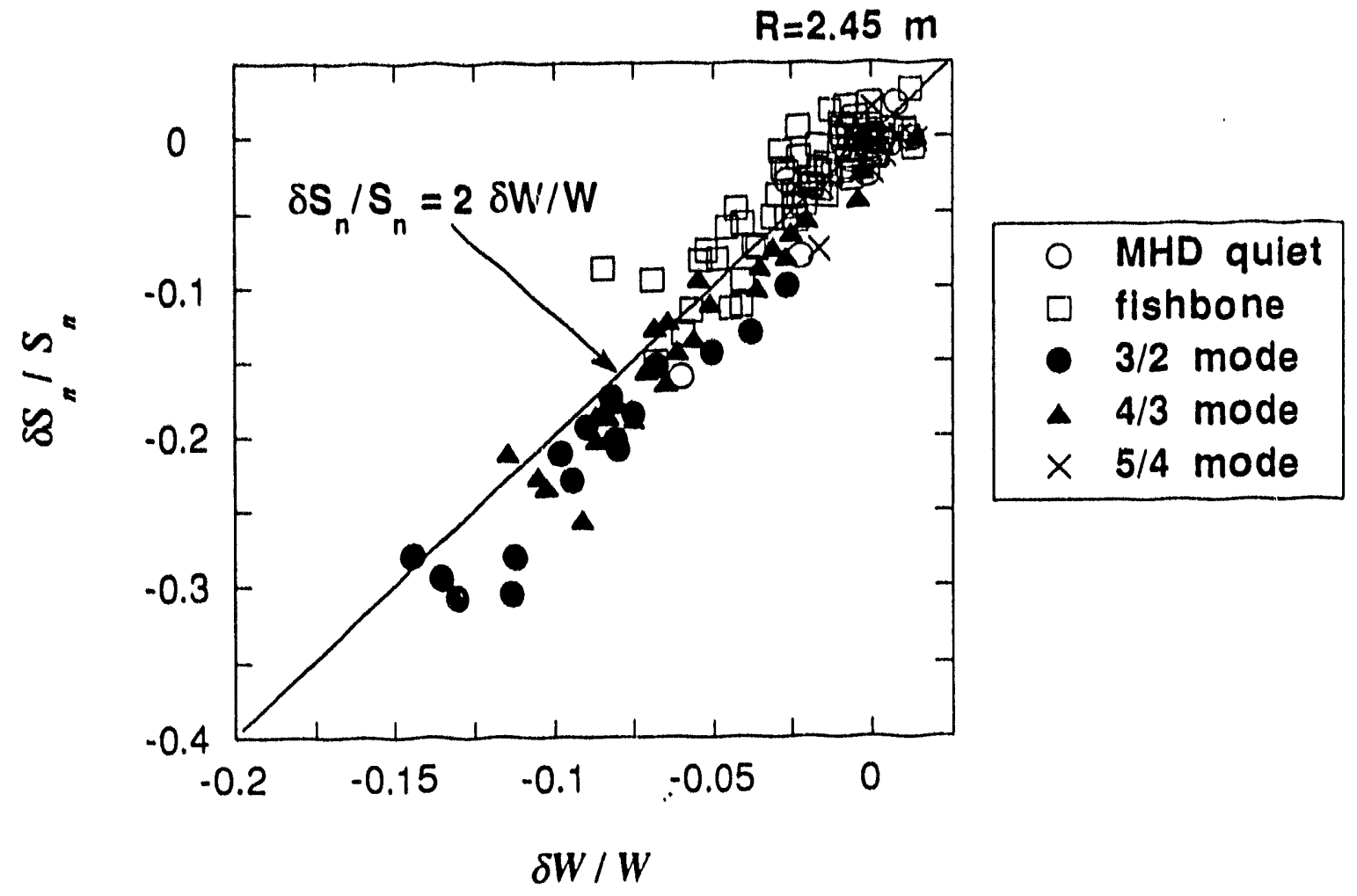

Figure 8: Relation between the neutron yield deterioration and energy deterioration, where the $\delta W / W$ is the relative change in plasma diamagnetic stored energy between its peak time and the time near the end of the beam phase. 


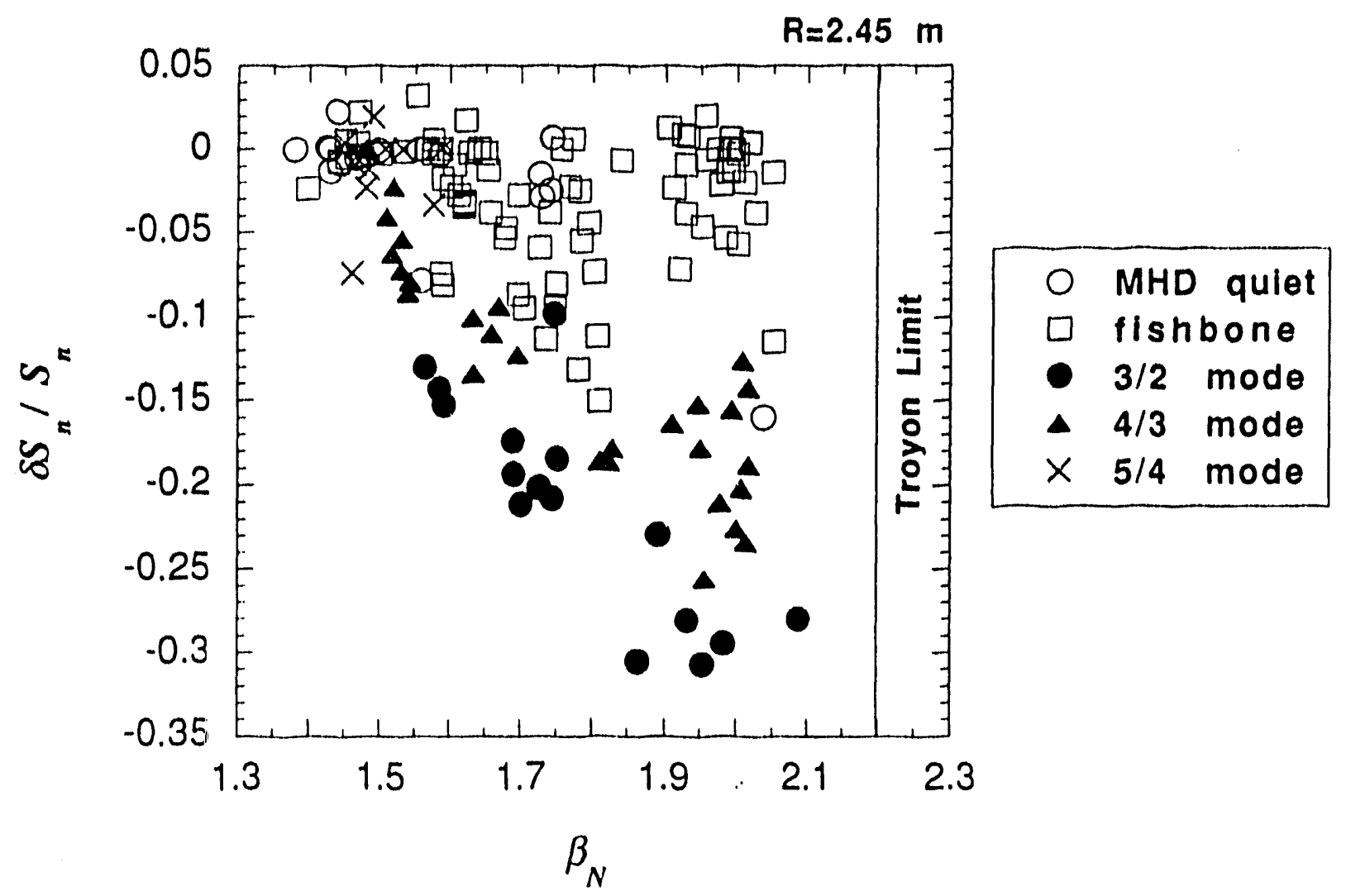

Figure 9: The neutron yield deterioration $\delta S_{n} / S_{n}$ versus the normalized toroidal, $\beta_{N} \equiv \beta_{t}(\%) a(m) B_{T}(T) / I_{p}(M A)$ for the same database as shown in Fig. 6. Here, $\beta_{t}$ is the volume averaged toroidal $\beta$ deduced from the magnetic measurement. The appearance of the coherent MHD modes does not relate to the nearness to the Troyon limit $\left(\beta_{c}^{\text {Troyon }} \sim 2.2\right)$. 


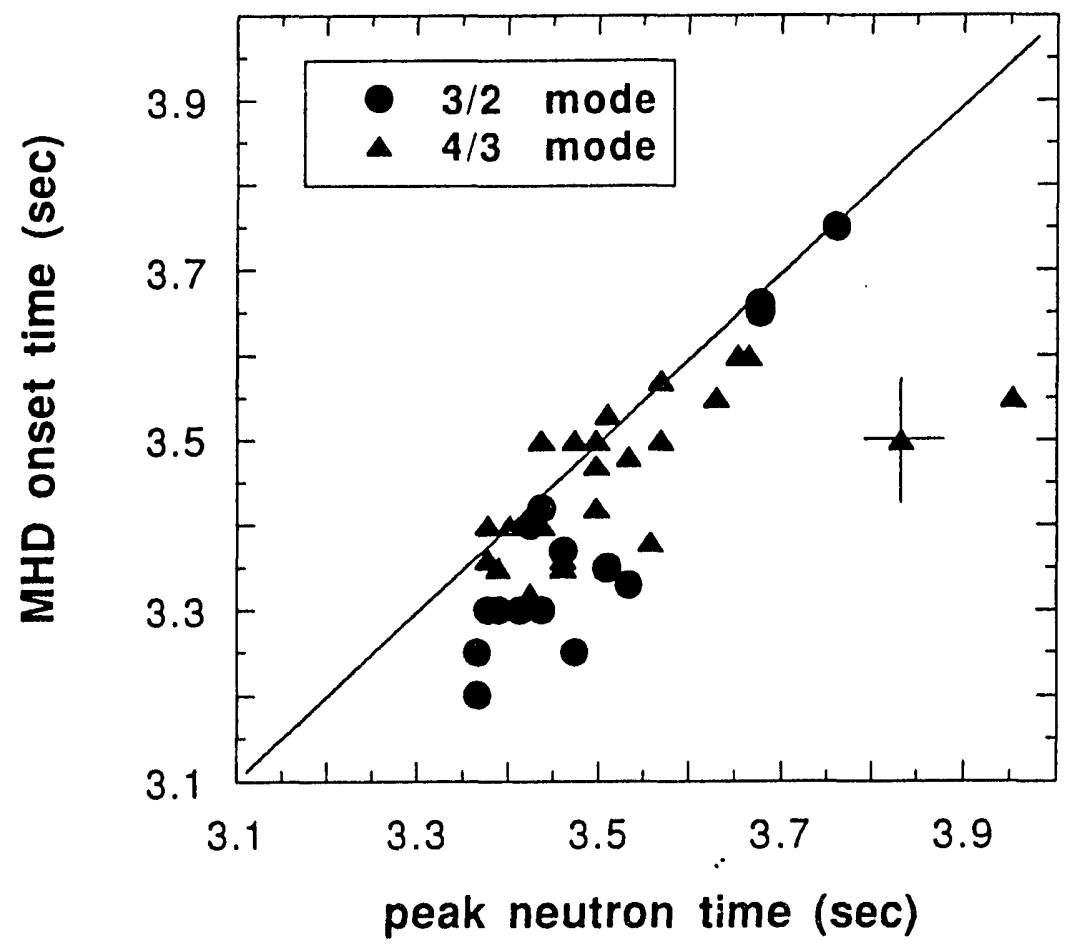

Figure 10: Correlation between the onset of MHD (3/2 and 4/3 modes) and the peak neutron yield time. Within the measurement error bars, the MHD always starts around or prior to the time of peak neutron yield. 


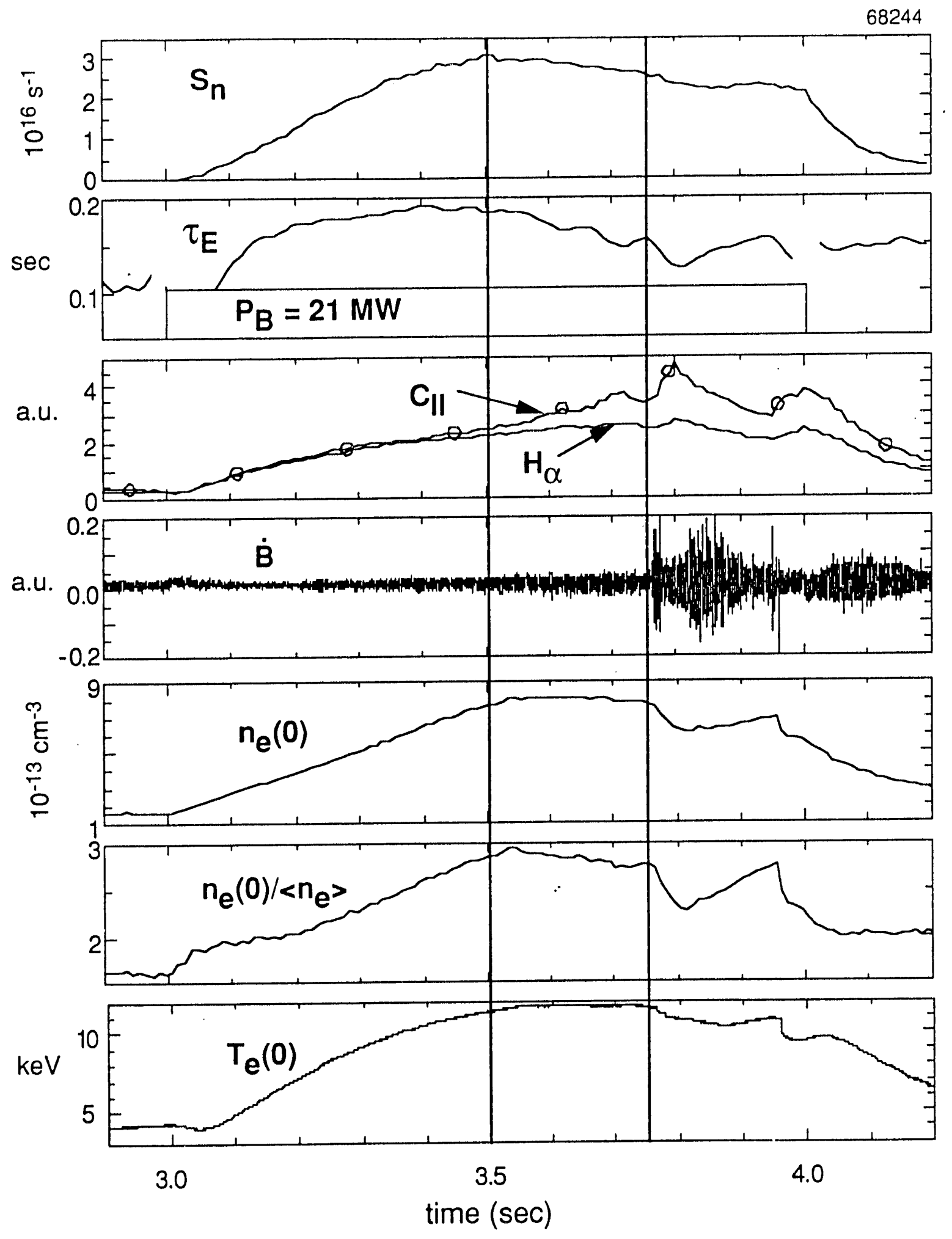

Figure 11: Performance deterioration phenomenon in the exceptional discharge (68244). Note that the neutron yield starts to decrease at $\sim 3.5 \mathrm{sec}$. No strong coherent MHD activity was detected until $\sim 3.75 \mathrm{sec}$. The possible candidates for the deterioration are the slow rise of the $C_{I I}$ and decrease of the electron density peakedness. 


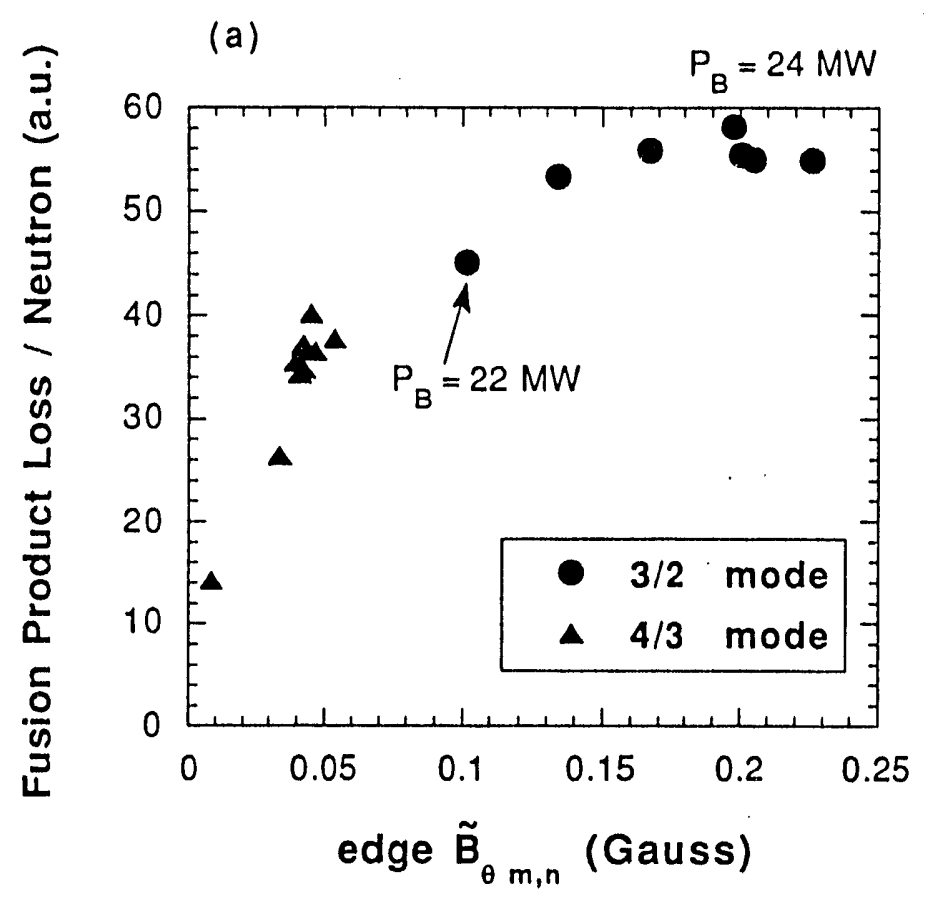

(b)

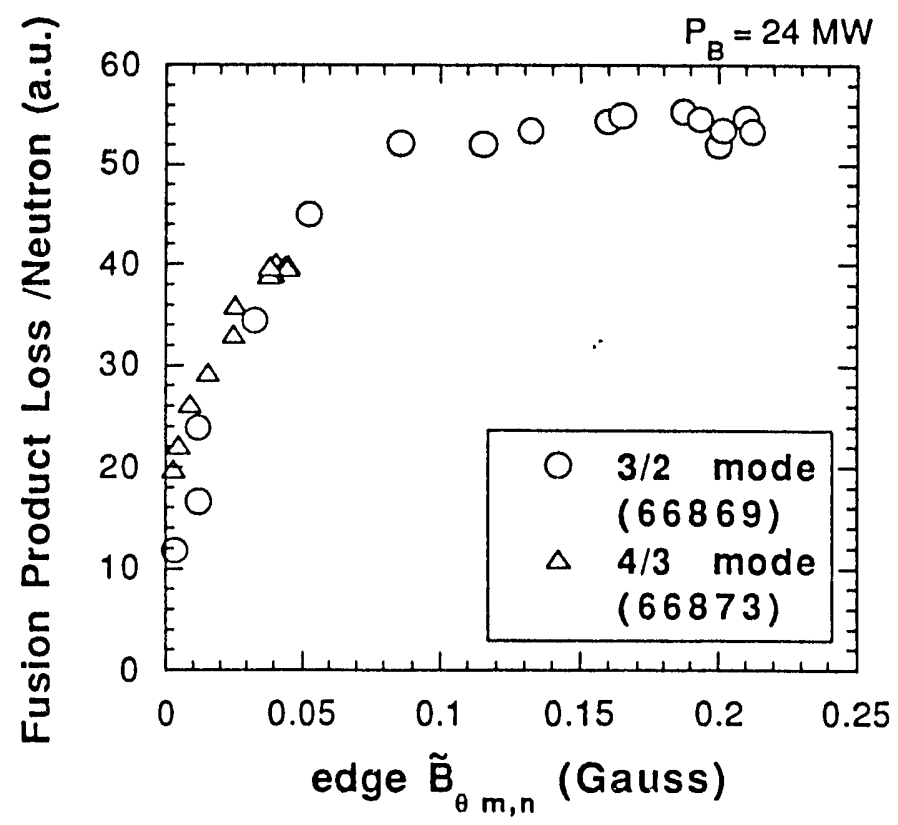

Figure 12: MHD induced fusion product particle loss. (a) The measured loss versus $\tilde{B}_{\theta m, n}$ for a subset of similar discharges from Fig. 7 with $3 / 2$ or $4 / 3$ MHD modes, where $\tilde{B}_{\theta m, n}$ is the dominant MHD mode amplitude measured at the edge. The measurement is taken at $\sim 0.7 \mathrm{sec}$ after the NBI. (b) Time correlation analysis for two discharges with either a dominant $3 / 2$ mode or $4 / 3$ mode. The data is taken at every $50 \mathrm{~ms}$ from the starting of the mode until the end of the NBI. Both analyses suggest a strong depletion of the fusion product population when the MHD amplitude exceeds 0.1 Gauss. 


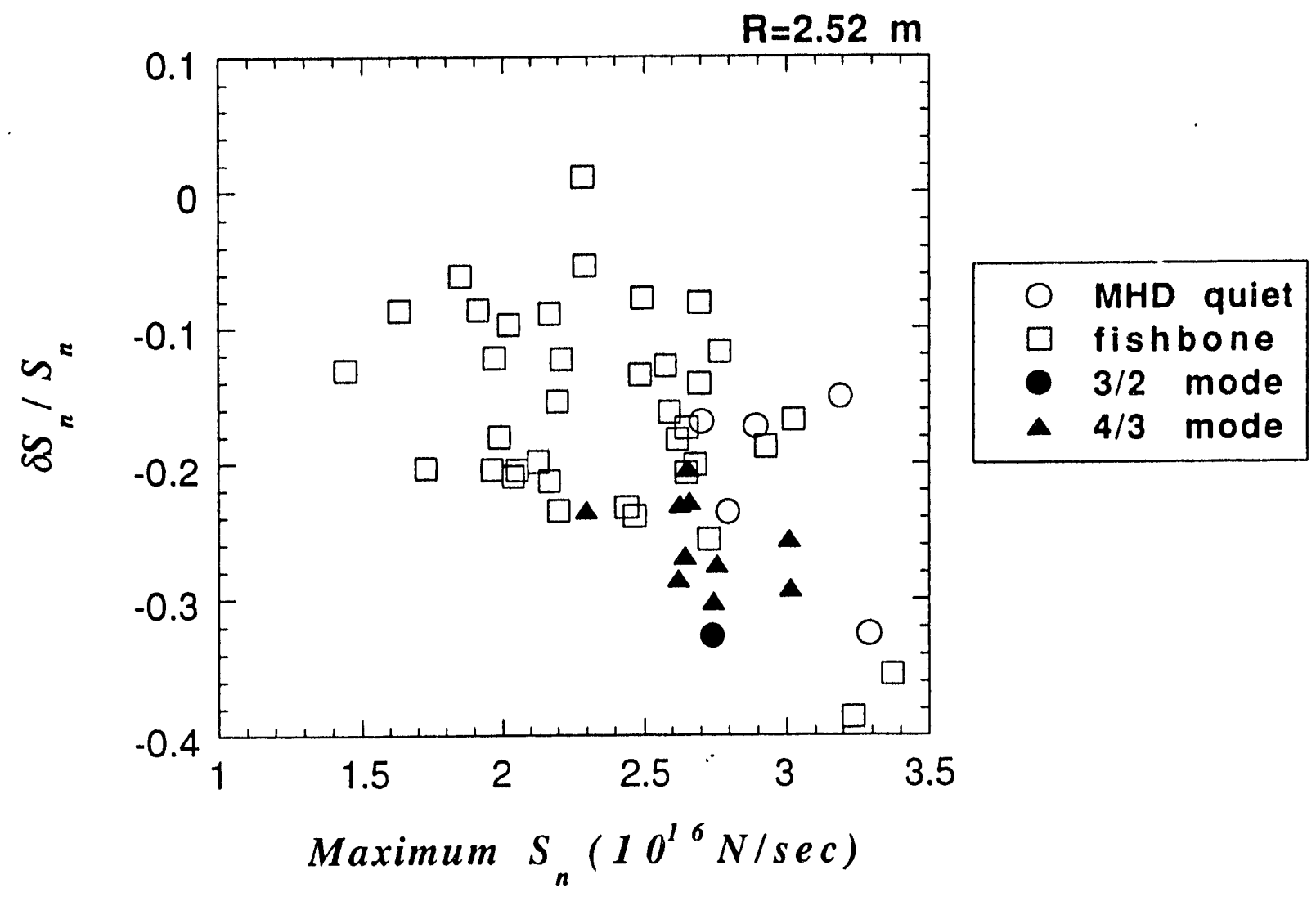

Figure 13: Statistical data analysis of the neutron yield deterioration for the $R \simeq$ $2.52 \mathrm{~m}$ plasmas. 


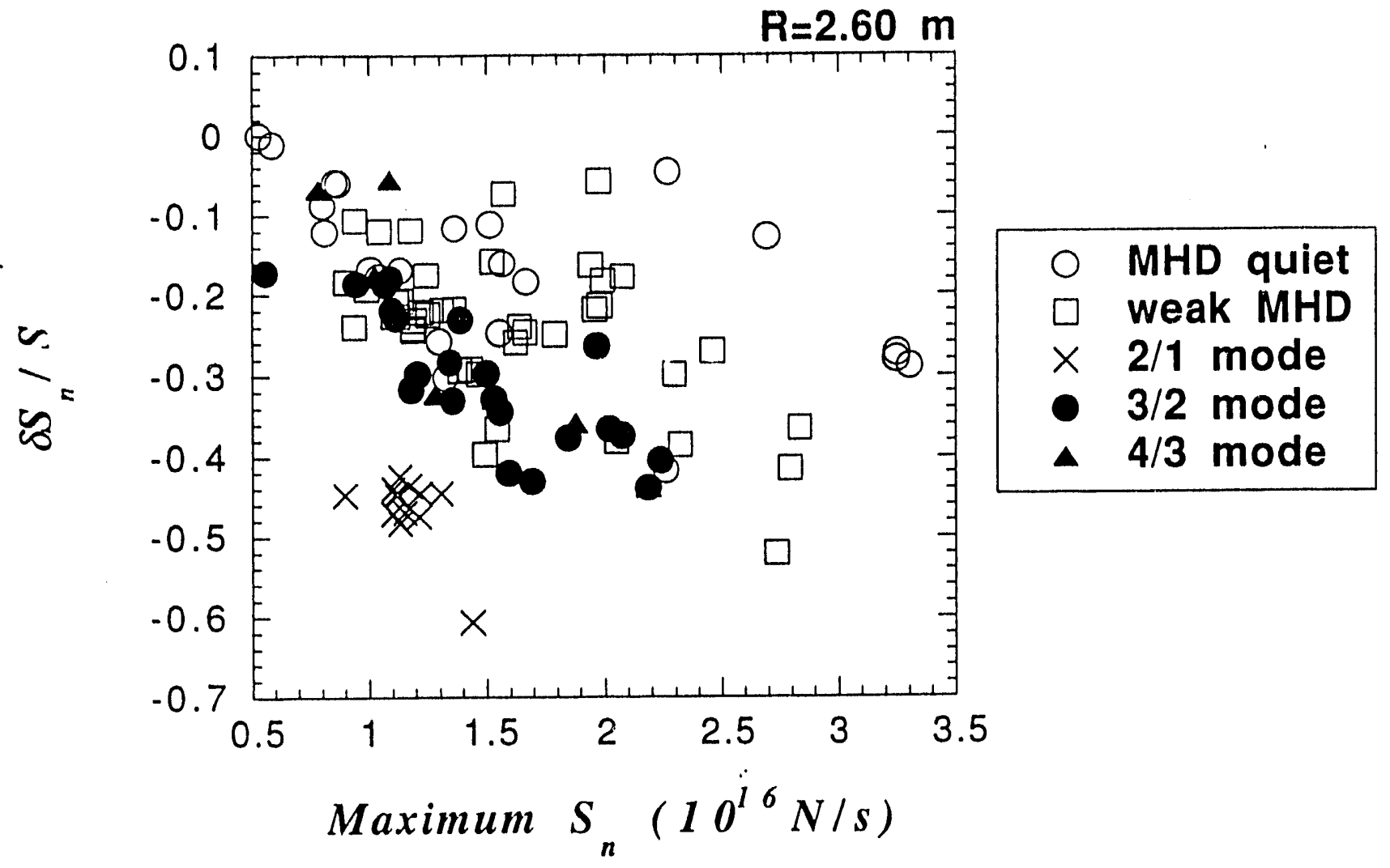

Figure 14: Statistical data analysis of the neutron yield deterioration for the $R \simeq$ $2.60 \mathrm{~m}$ plasmas. Here, the "weak MHD" represents the situation in the MHD analysis around the peak neutron yield time - there are no strong coherent modes detected from Mirnov system, but some internal mode structures may be seen on the ECE diagnostic. 


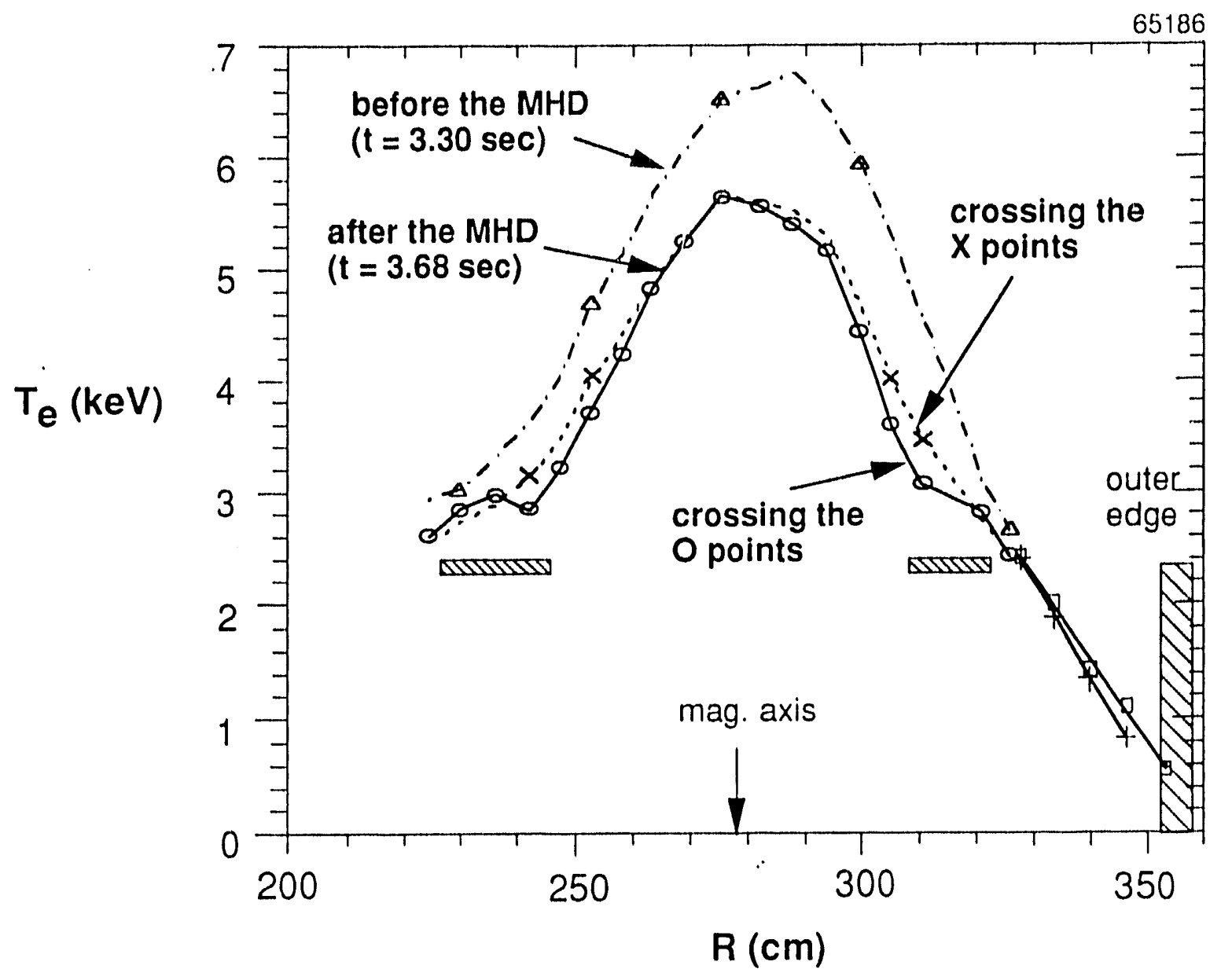

Figure 15: The $T_{e}(R)$ evolution and mode structure of a discharge with an $m / n=$ $2 / 1$ mode measured by the ECE grating polychromator diagnostic. The mode starts at $\sim 3.4 \mathrm{sec}$. A significant drop in $T_{e}$ is observed in the core inside the mode rational surface (island region). Not much change is observed outside the mode rational surface. The island structure can be seen from the two $T_{e}$ profiles crossing the $O$ and $X$ points of the island. The $T_{e}$ profile outside $325 \mathrm{~cm}$ is from the ECE Michelson interferometer diagnostic [13]. 


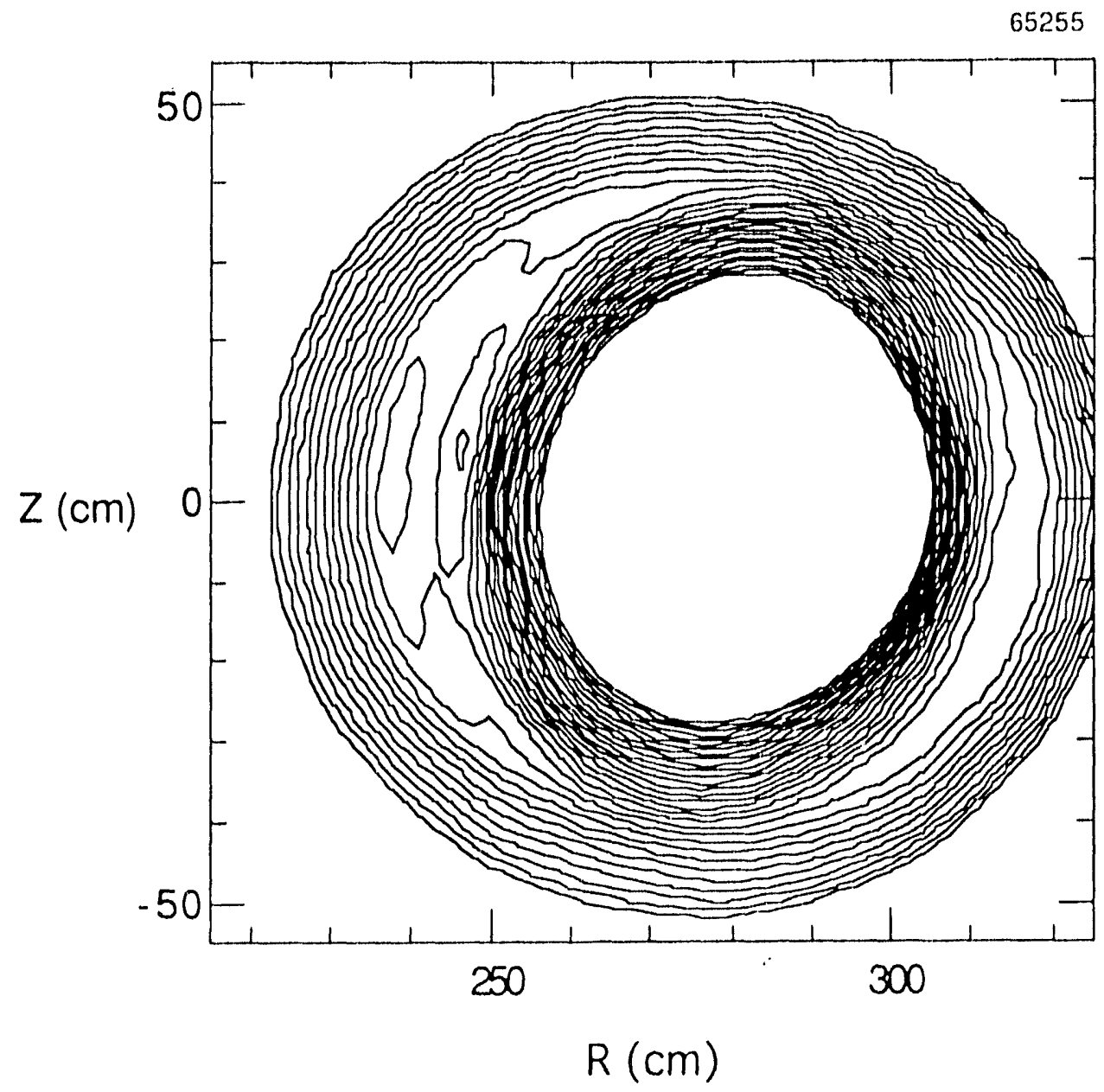

Figure 16: The 2-D $T_{e}$ contour plot of the 2/1 temperature island from a discharge similar to Fig. 15. 

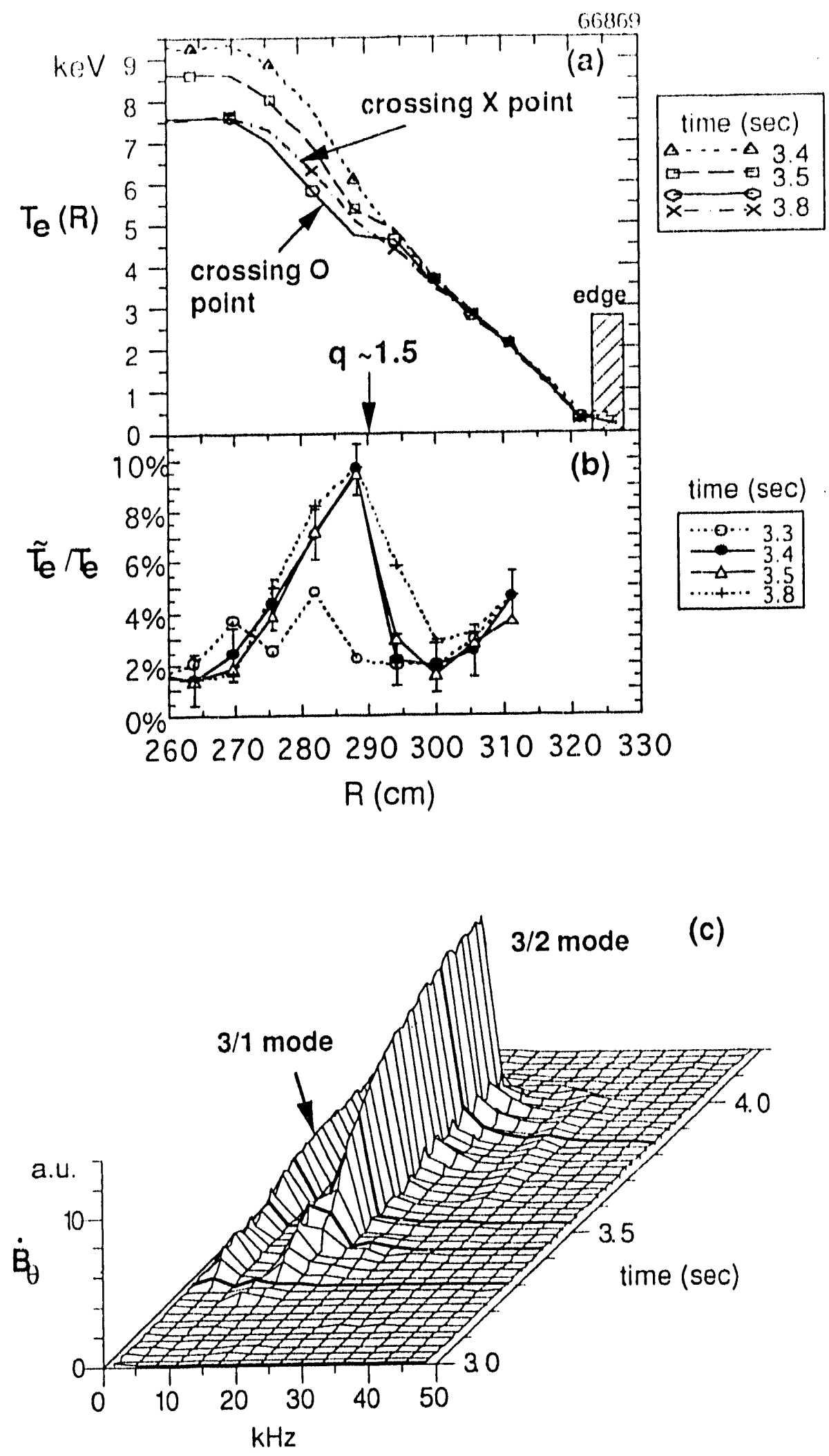

Figure 17: The effects of an $m / n=3 / 2$ MHD mode on the cvolution of plasma profiles. (a) The $T_{e}$ profiles at three different stages of the mode evolution highlighted on the $\dot{B}_{\theta}$ frequency spectrum in (c). The island structure (flat spot) can be identified from the $T_{e}(R)$ around the mode rational surface. (b) The temperature fluctuations at the four different times. The center of the outer-most magnetic flux surface is at $2.45 \mathrm{~m}$. An $m / n=3 / 1$ kink-like mode is also detected in this discharge. 

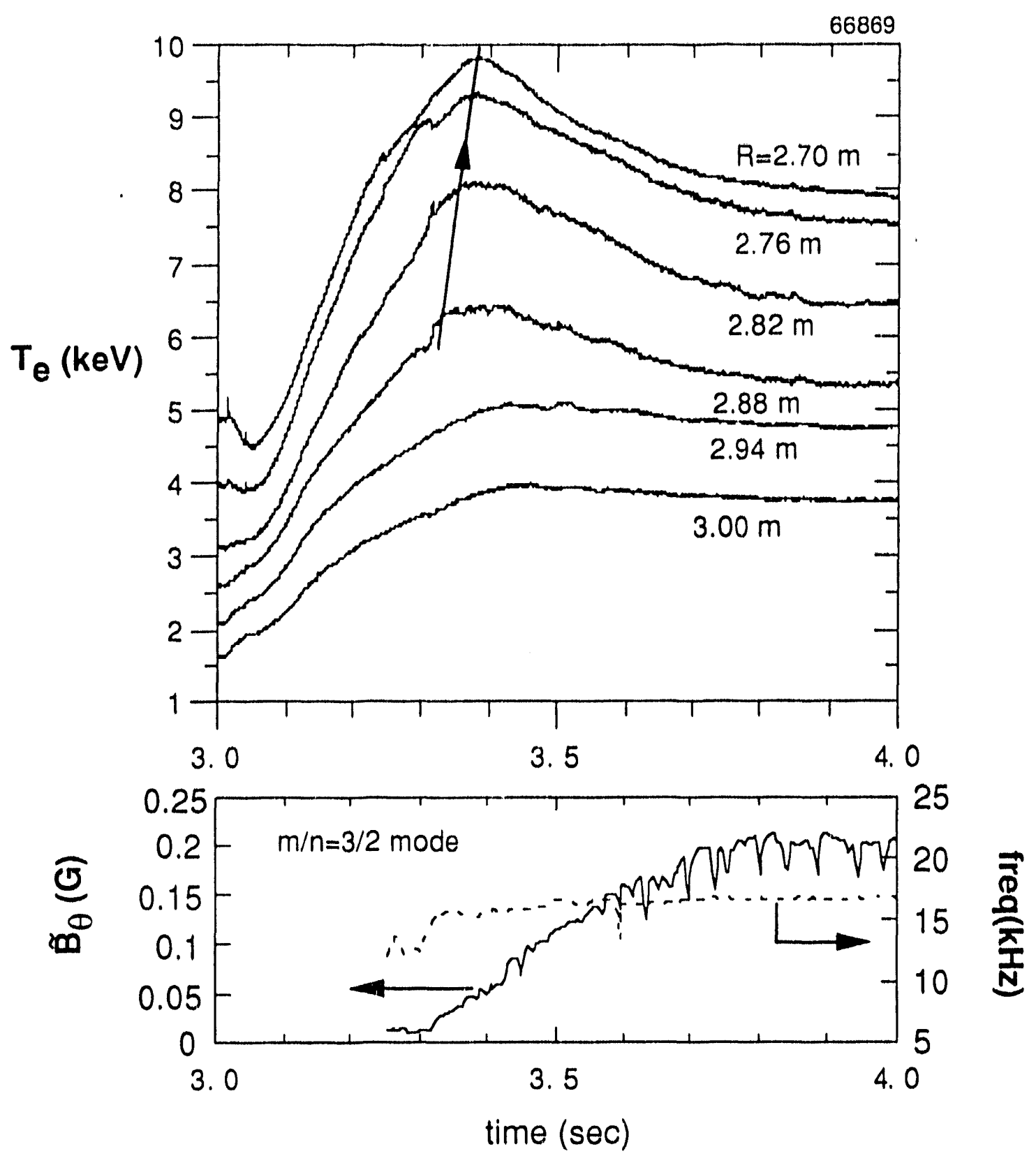

Figure 18: Time evolution of the $T_{e}$ at different radii for the same discharge as shown in Fig. 17. The effect of the $3 / 2$ mode "propagates" to the plasma center in $<50 \mathrm{msec}$. Then, a slow decay of the $T_{e}$ profile inside the mode rational surface followed in $\sim 300 \mathrm{msec}$ correlated with the slow rising of the $3 / 2$ mode shown on the lower plot. The mode frequency does not change much during the whole evolution. 

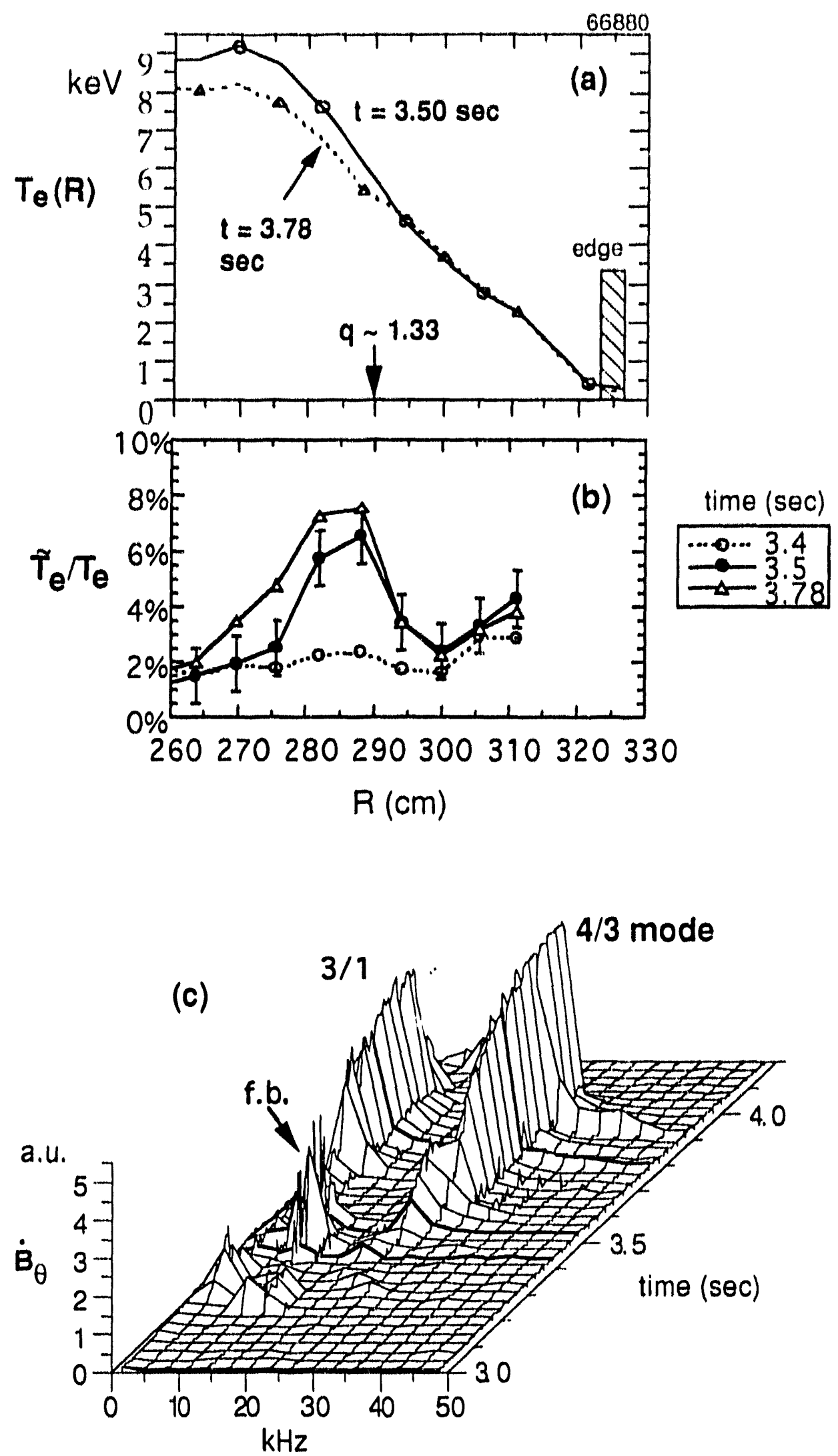

Figure 19: Similar data analysis as Fig. 17. In this discharge the dominant MHD mode detected is an $m / n=4 / 3$ mode started at $\sim 3.5$ sec. A "flat spot" can be almost seen on $T_{e}(R)$. A fishbone-type MHD activity is observed before the $4 / 3$ mode. 

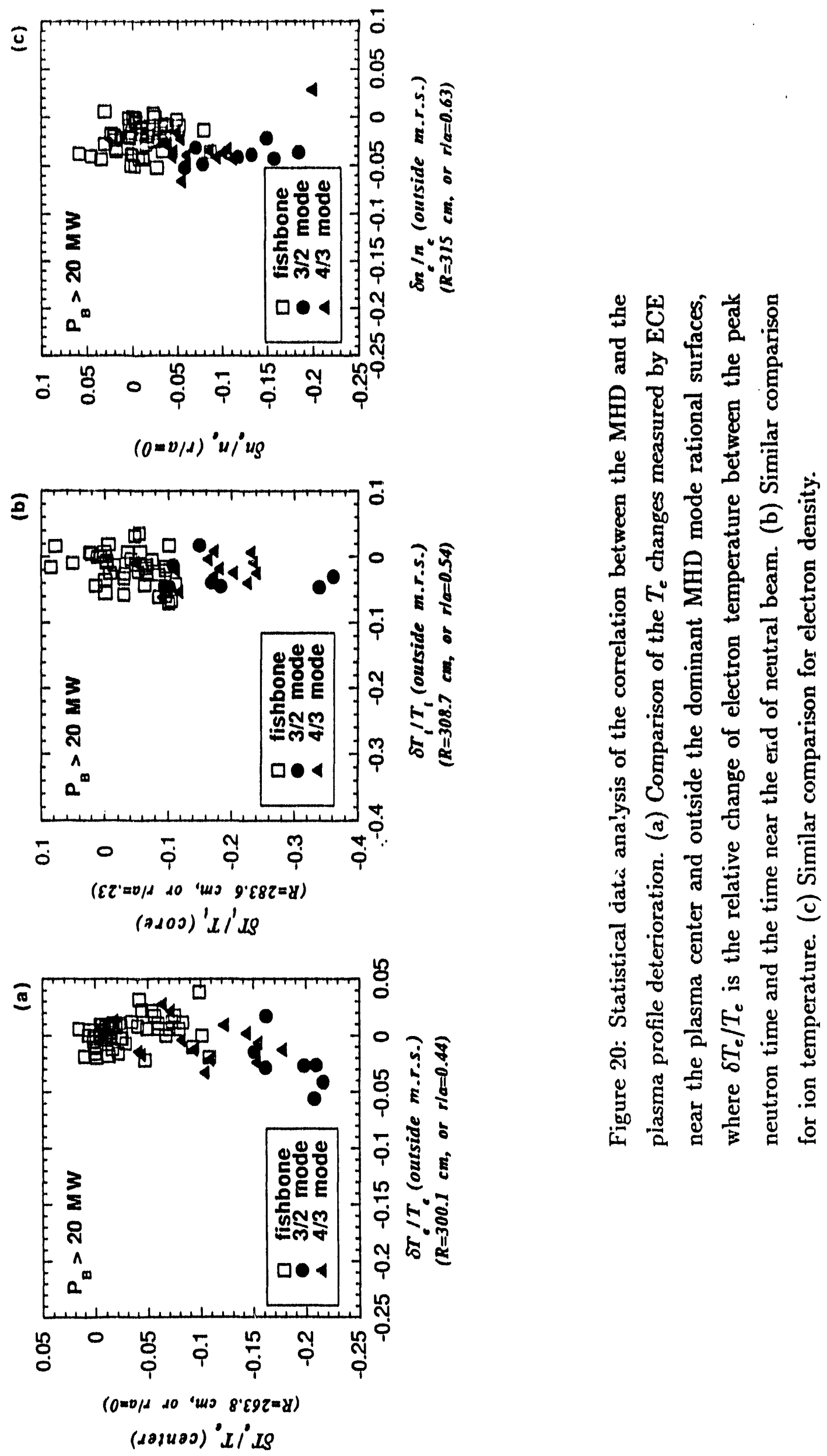


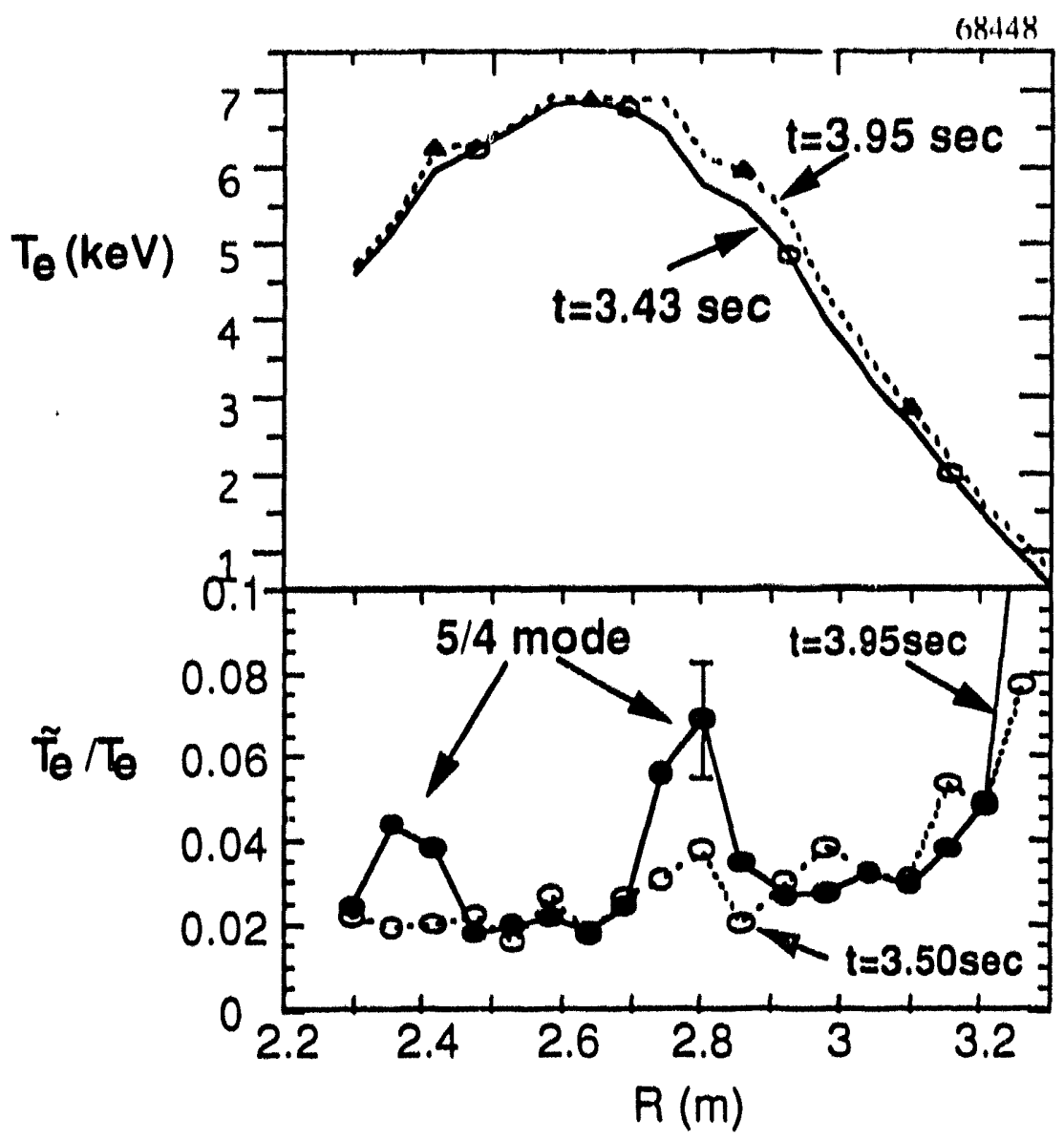

(a)

(b)

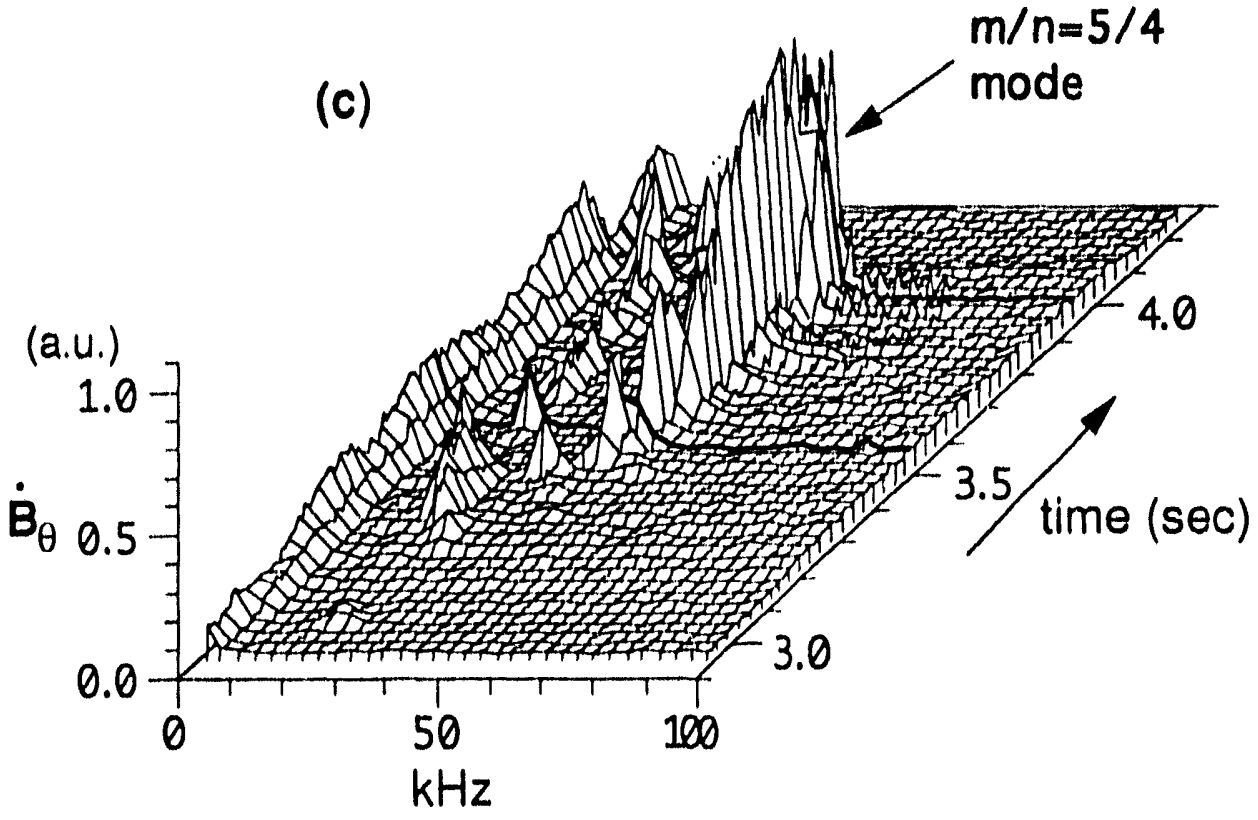

Figure 21: Similar data analysis as Fig. 17 for a supershot with a dominant $m / n=$ $5 / 4$ mode. (a) The electron temperature profiles at two different times, $3.43 \mathrm{sec}$ (before the start of the $5 / 4$ mode) and $3.95 \mathrm{sec}$ (at the mode saturation phase). (b) The profiles of the $T_{e}$ fluctuation around the two times. (c) The MHD spectrum during the NBI phase (3.0-4.0 sec). The two times are highlighted. 


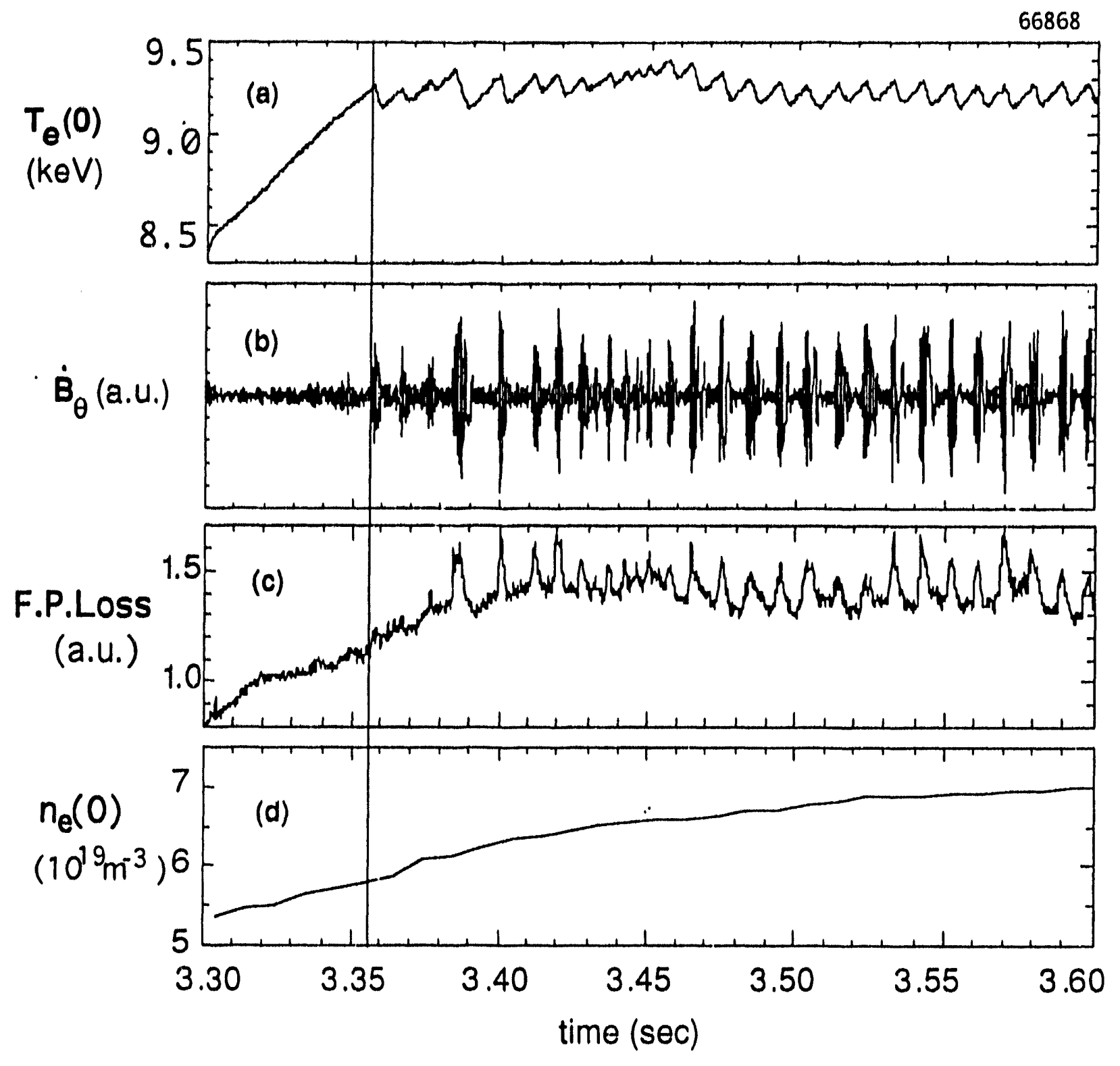

Figure 22: Effect of the fishbone-type MHD mode on the central electron temperature evolution. After the onset of the mode in (b), the central $T_{e}$ (a) exhibits a departure from its increasing phase and sawtooth like oscillations afterward. The fusion product loss also shows the corresponding bursts with $\sim 10-20 \%$ enhancement for each fishbone burst. In contrast, the electron density does not seem to be affected by the fishbone-type mode. 


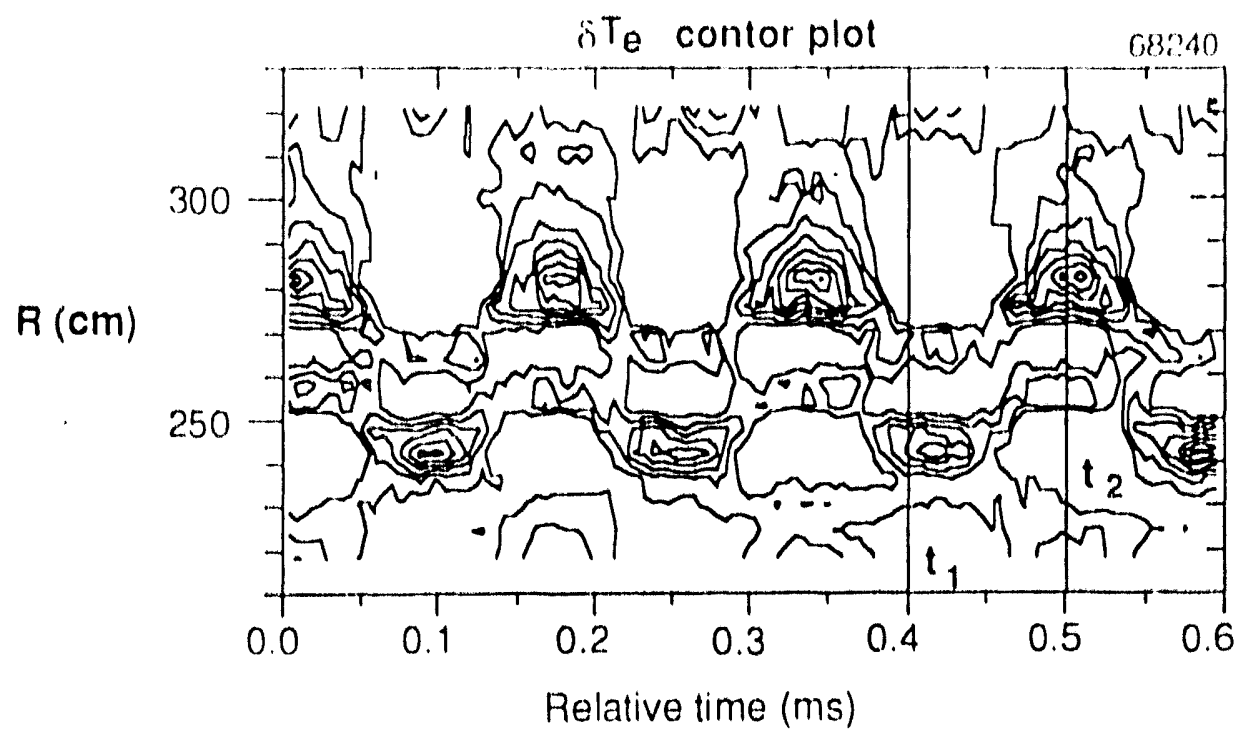

(a)

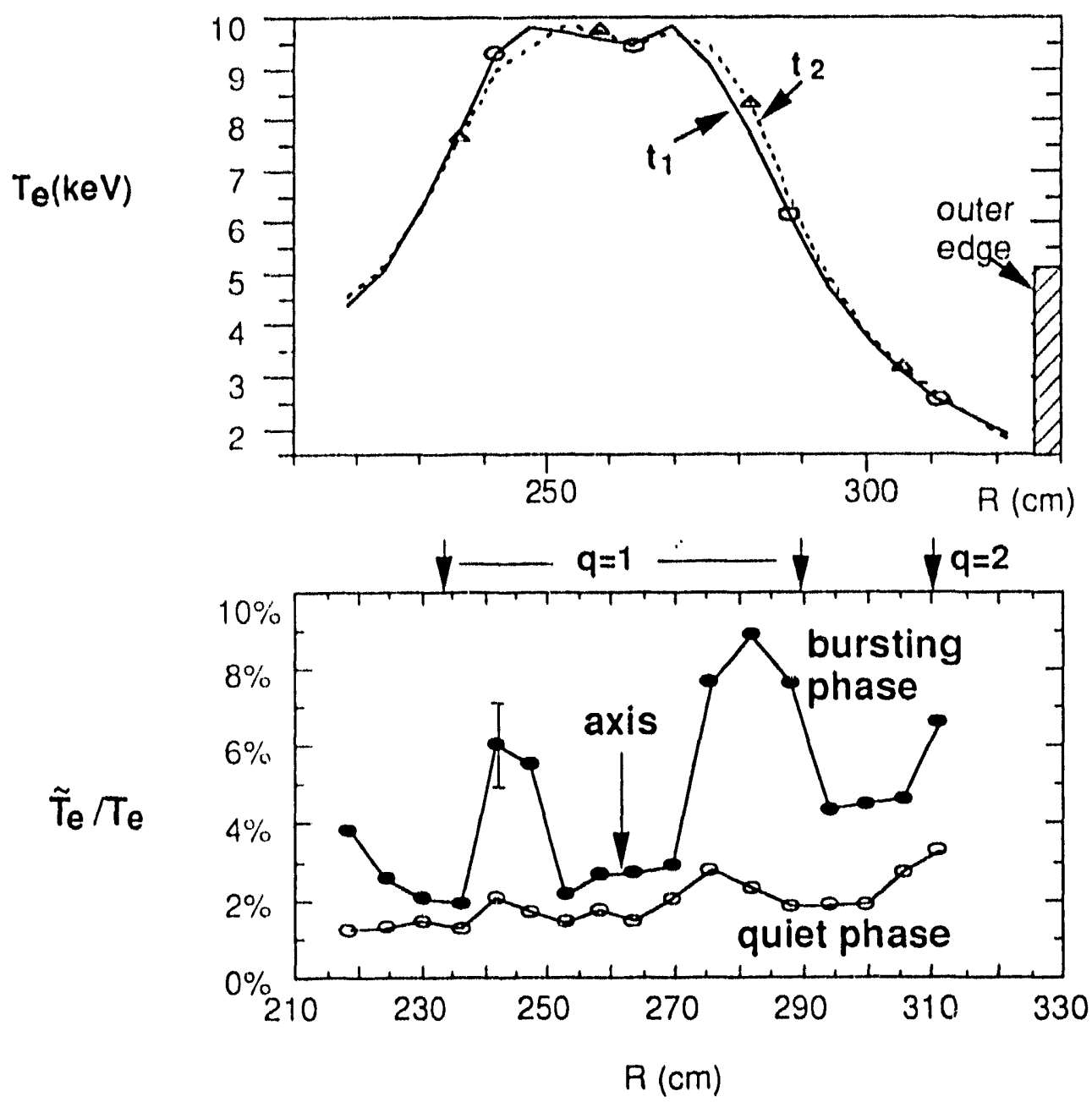

(b)

(c)

Figure 23: (a) (ontour plot of the 87 , versus time. $A$ clear $m / n=1 / 1$ oscillation is seen. (b) Oscillation of the core $T_{e}$ profile during the fishbone bursting phase. The two profiles correspond to the two different times shown in (a). (c) Electron temperature fluctuations measured by ECE for the two phases of the fishbone-type MHD. A large increase of the fluctuations can be seen inside the $q=1$ surface. The burst also couples to outside higher $m$ surfaces. 


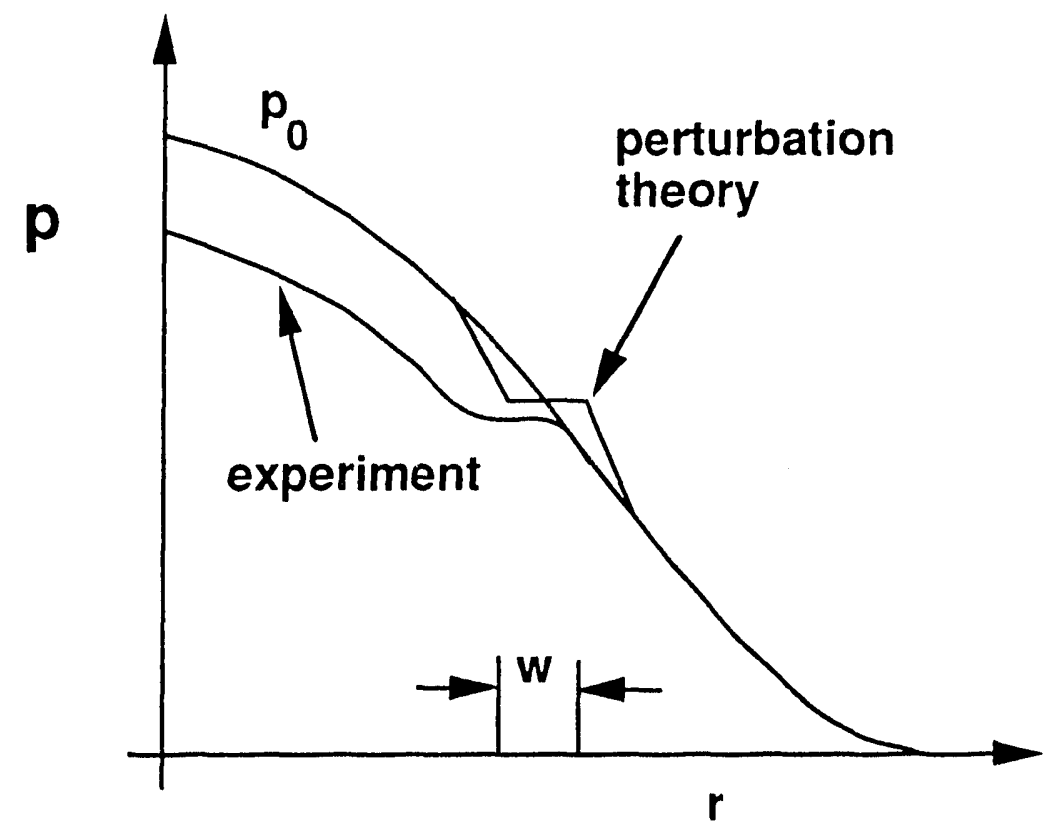

Figure 24: Illustration of the pressure profile change from perturbative tearing mode theory. Only a small amount of free energy is involved in the analysis, i.e., $\delta W / W \propto w^{4} / a^{4}$. On the other hand, the experimental measurement shows that the whole pressure inside the mode rational surface profile decreases after the onset of the island on the transport time scale. Therefore, the deterioration of energy is $\delta W / W \propto w / a$. 


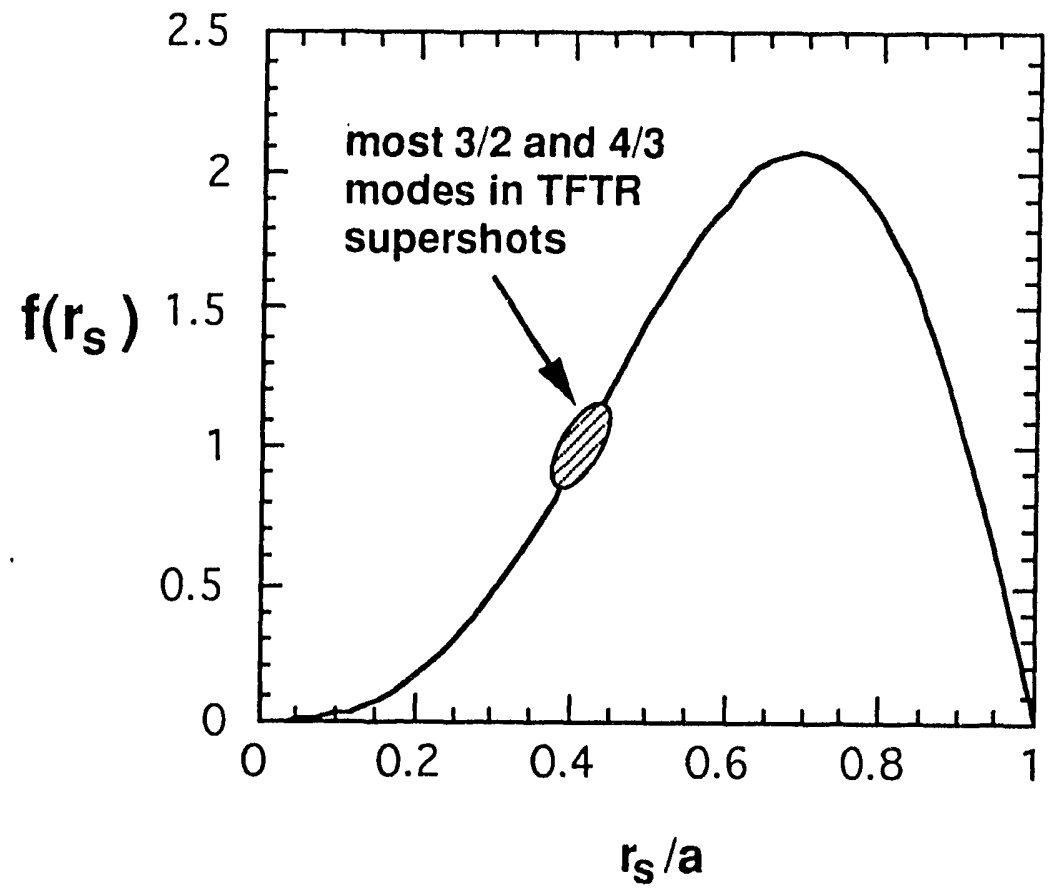

Figure 25: The energy deterioration coefficient $f\left(r_{s}\right)$ given by Eq. (6). For most of the $3 / 2$ and $4 / 3$ modes observed in supershot plasmas, this factor is $\gtrsim 1$. 
(a)

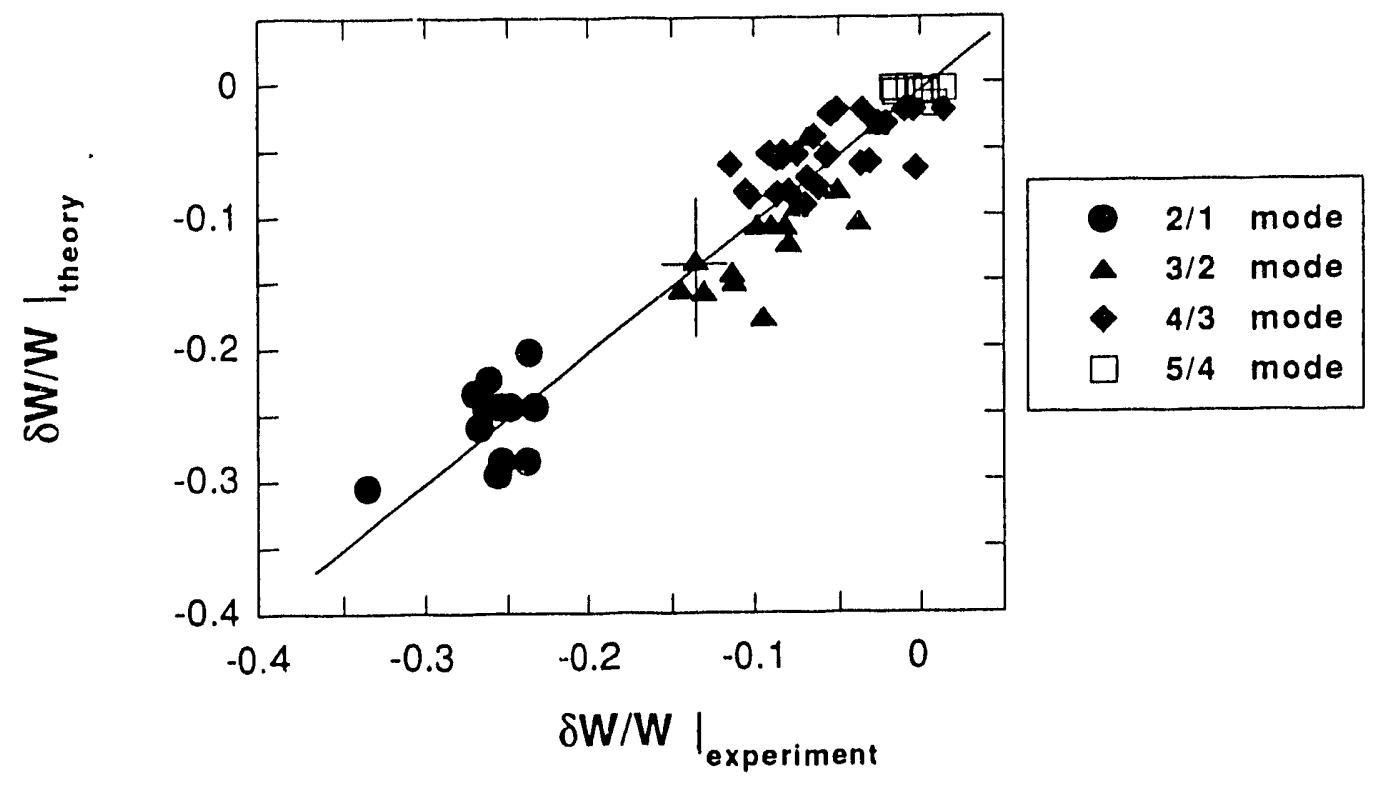

(b)

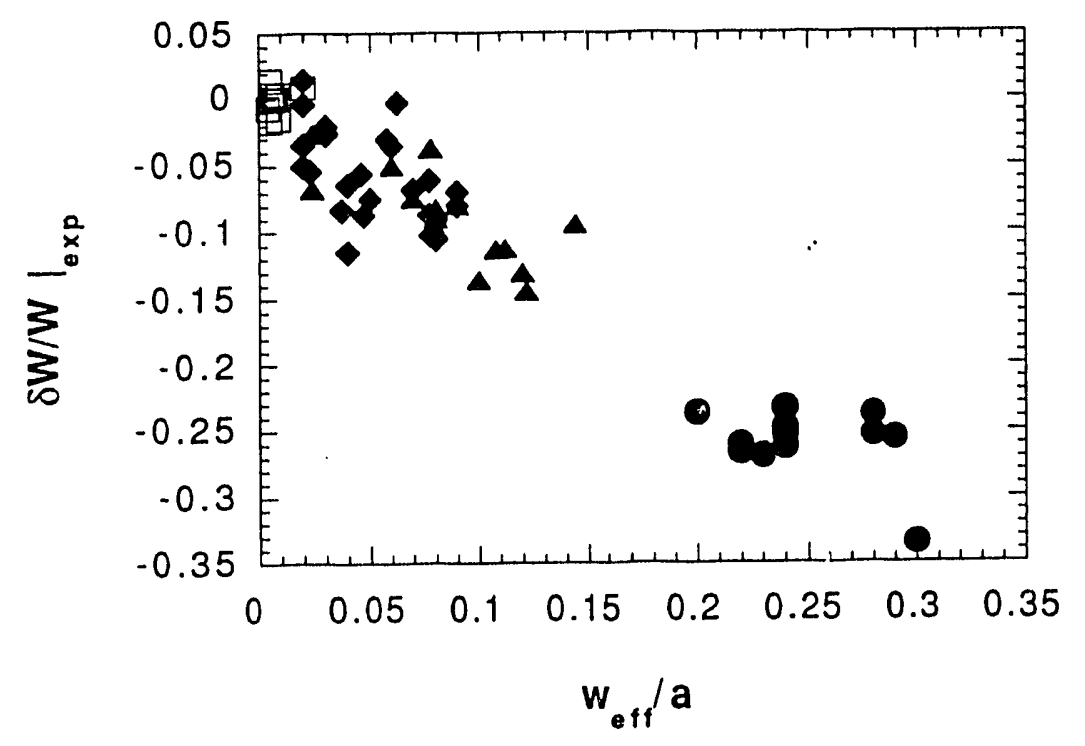

Figure 26: (a) Comparison of the theoretical model in Eq. (5) with the experiment (same data as shown in Fig. 6). To extend the parameter regime, the 2/1 mode data from the $R \simeq 2.60 \mathrm{~m}$ discharges are included. The calculated island width is shown in (b). 


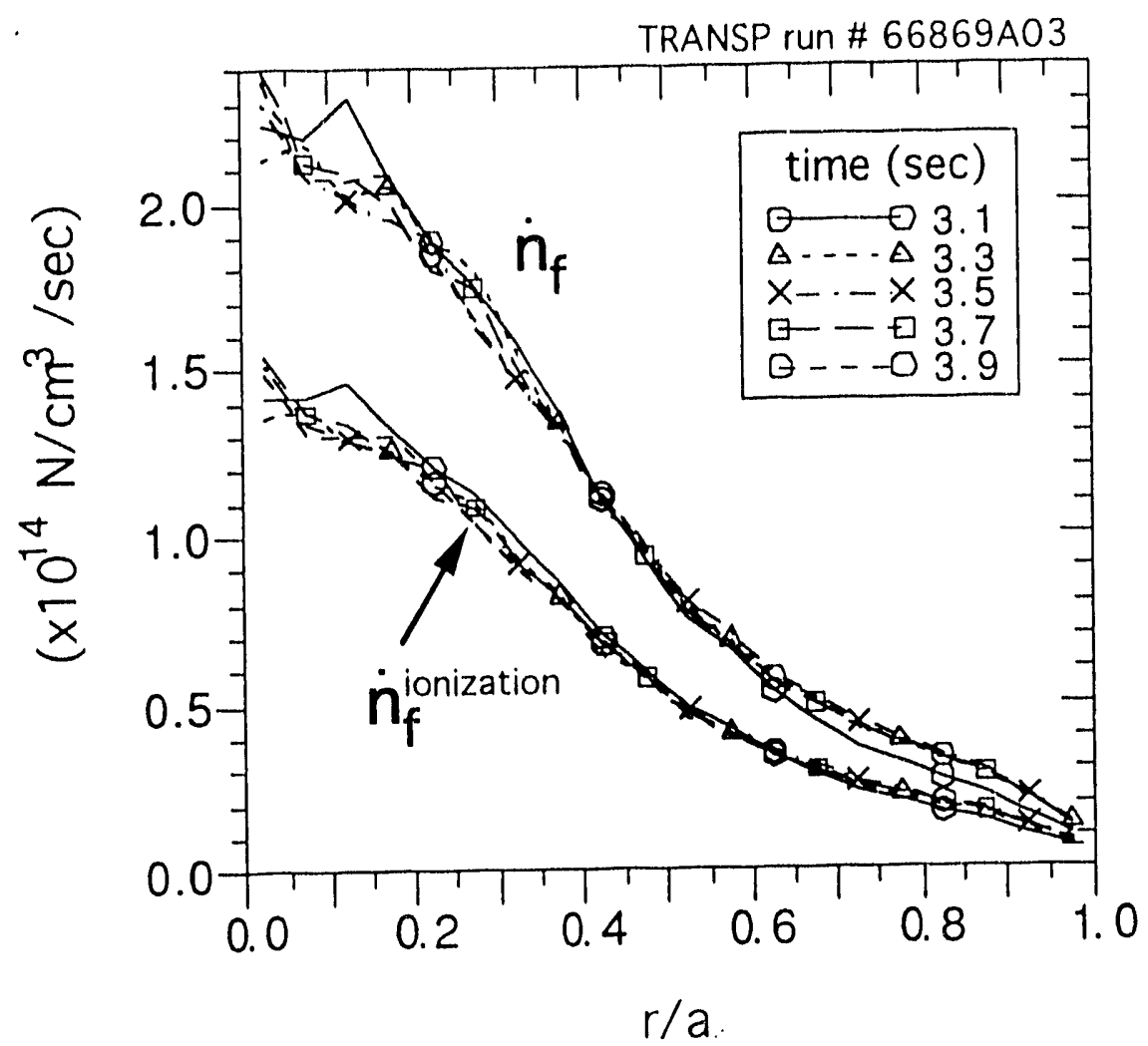

Figure 27: The TRANSP code calculated beam fueling rates. $\dot{n}_{f}$ is the total fueling rate which includes the main ionization and charge-exchange processes in the beam heated plasma. $\dot{n}_{f}^{\text {ionization }}$ is the total beam ionization rate. Note that in this discharge (66869) there is a strong $3 / 2$ mode that developed after $\sim 3.3 \mathrm{sec}$ (see Fig. 1). No changes, however in the beam fueling rates can be seen. 

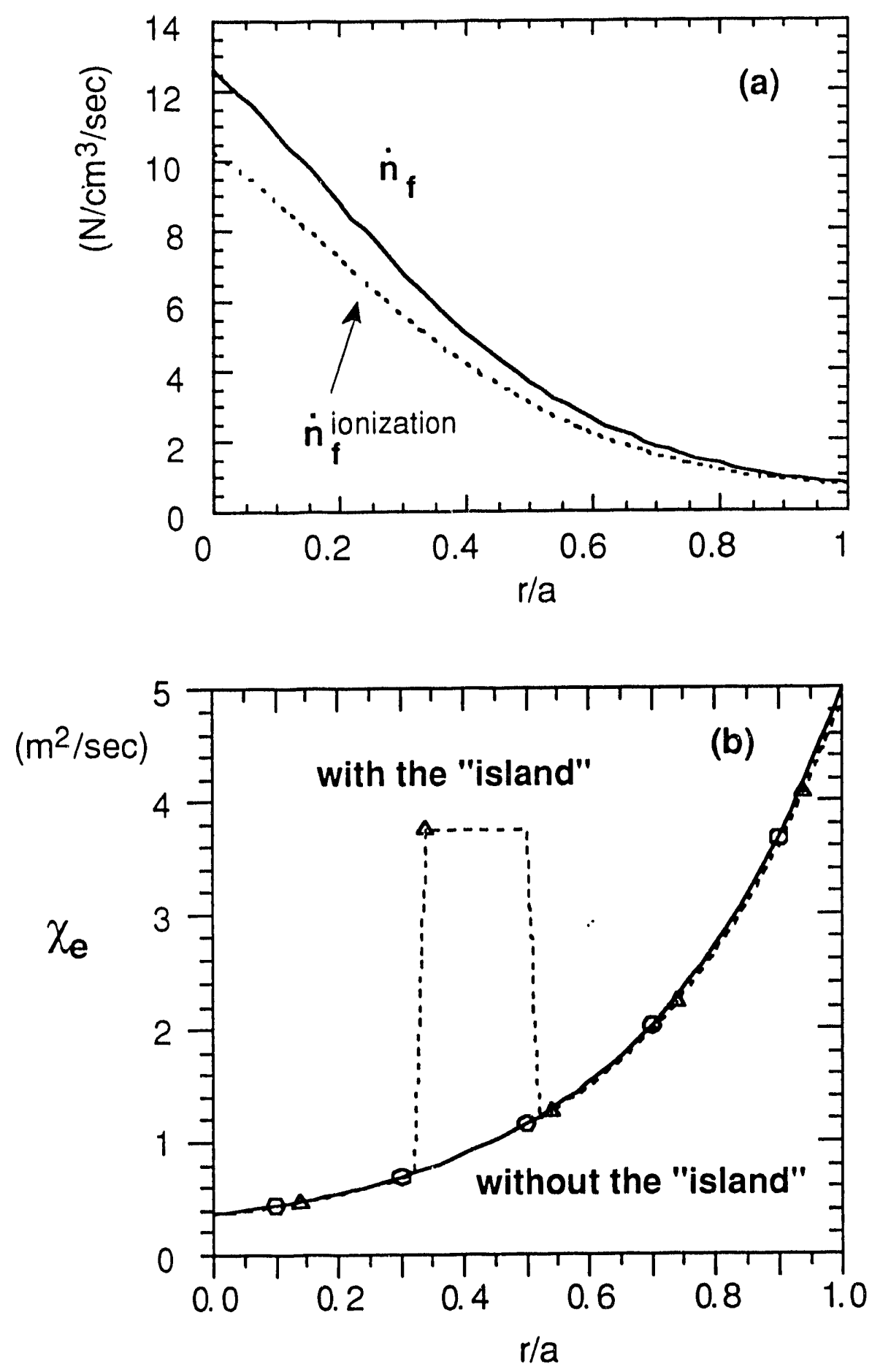

Figure 28: (a) The beam fueling sources used in the four-parameter simulation, where $\dot{n}_{f}$ is the total fueling rate (charge-exchange plus ionization) and $\dot{n}_{f}^{\text {ionization }}$ is the ionization part. (b) The modeled "magnetic island" effect on the effective electron heat diffusivity $\chi_{e}$ (and $\chi_{i}, D_{e}$ as well). An enhanced transport region around the mode rational surface is introduced after onset of the island. 
(a)

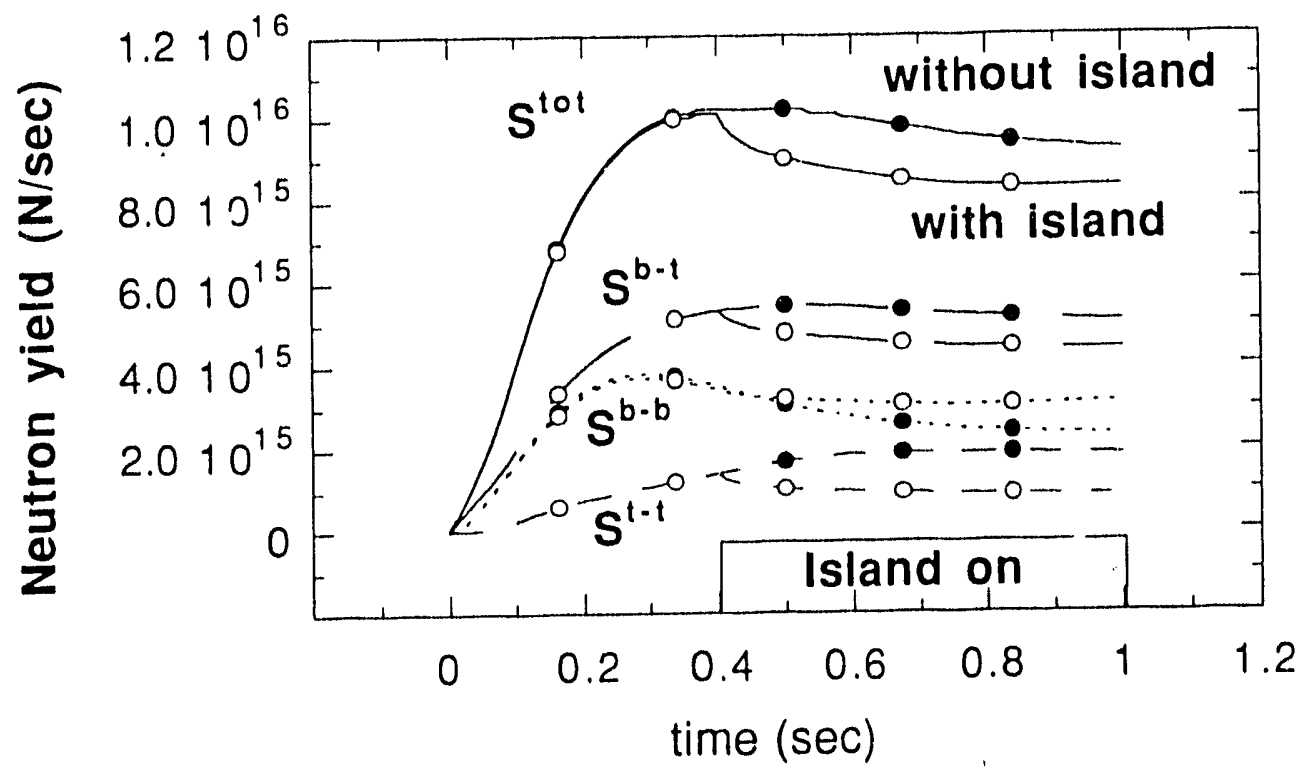

(b)



Figure 29: Simulation results. (a) The evolution of the simulated neutron rate in the NBI phase. The curves with solid dots are the simulation without turning on the island model. The curves with open dots are the results with the island model on from $0.4 \mathrm{sec}$ after the beam. (b) Simulated evolution of the total stored energy' and confinement time. 

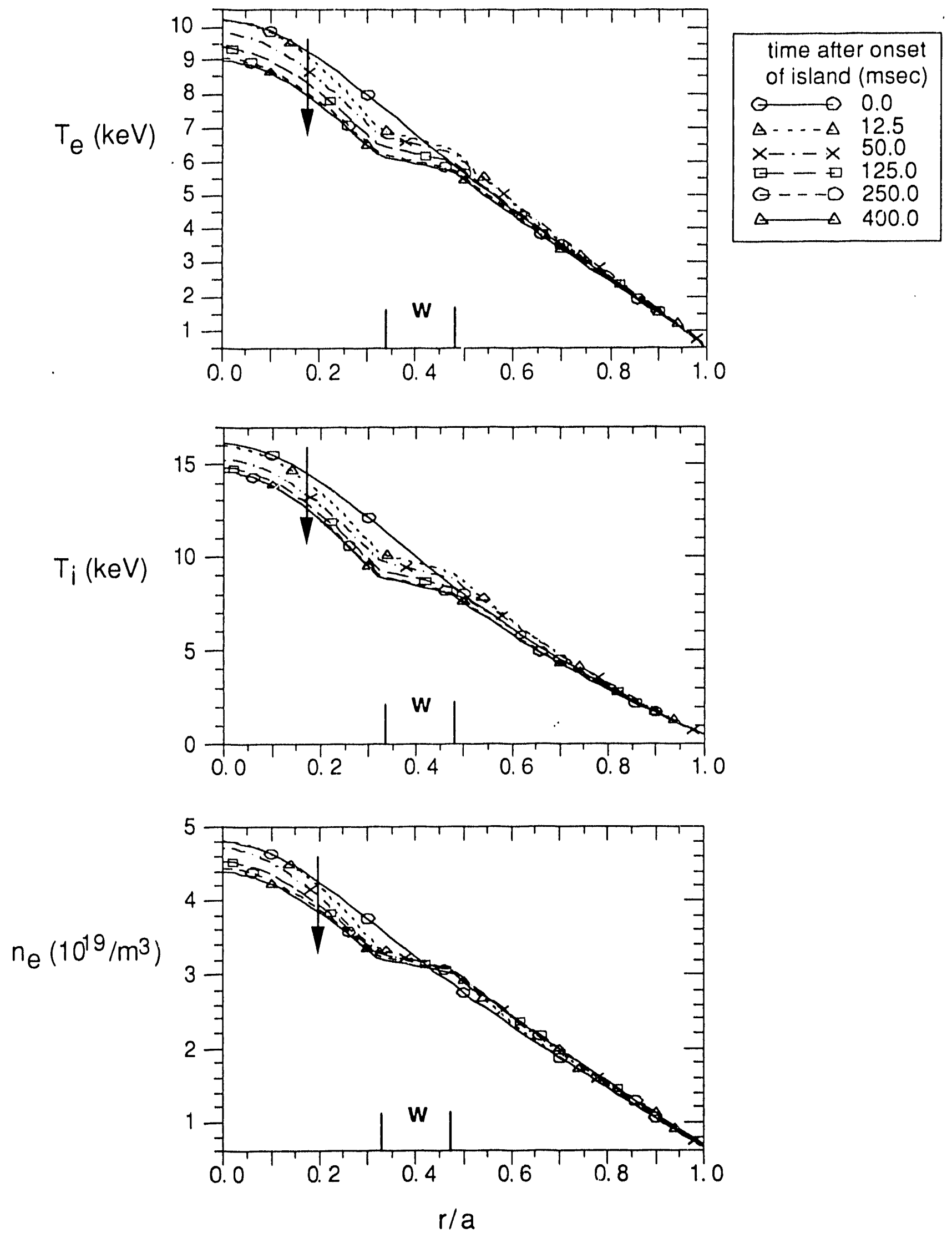

Figure 30: Time evolution of the simulated plasma profiles after onset of the island. 


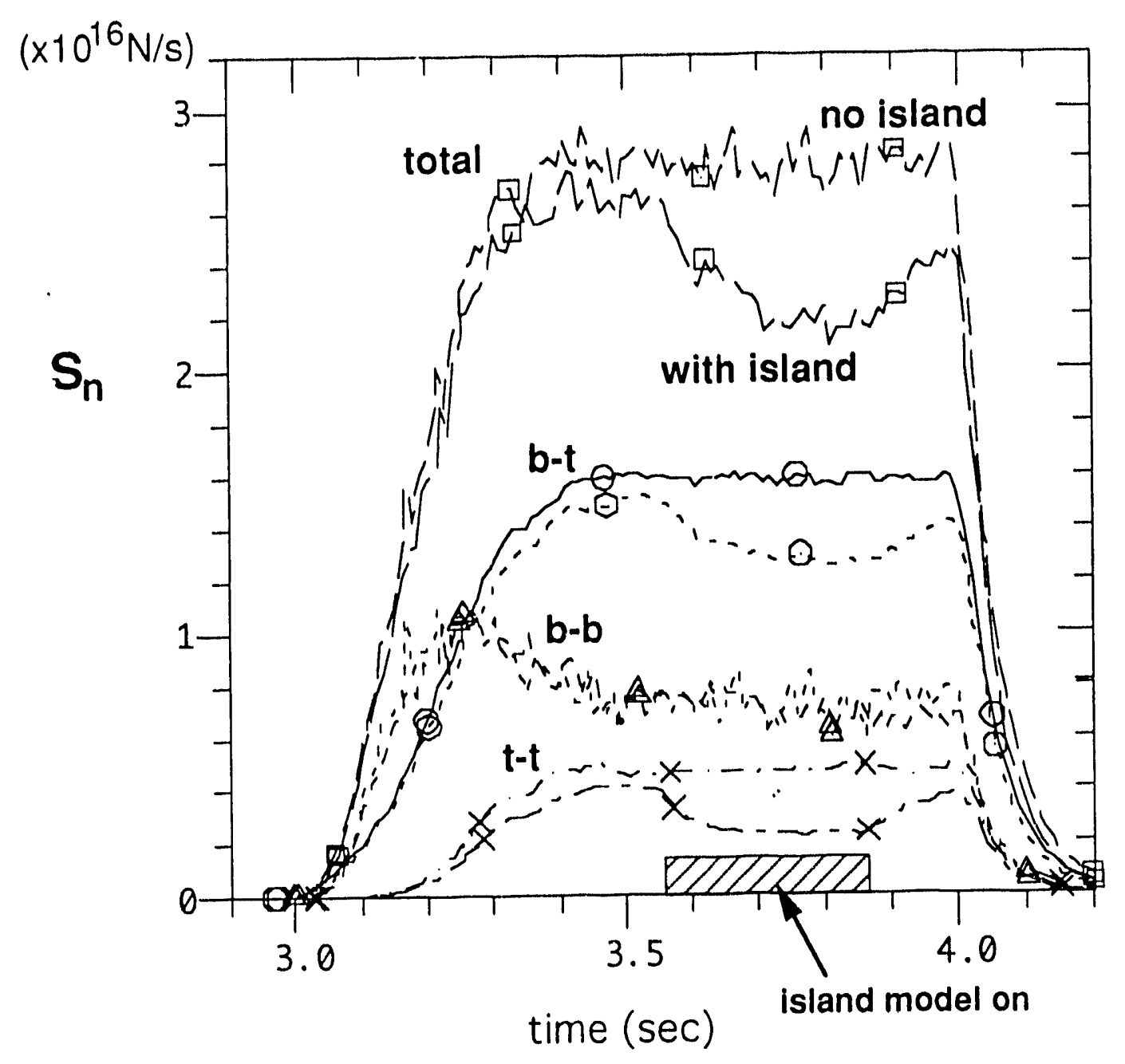

Figure 31: Simulated neutron yield evolution from the TRANSP code. The nondeteriorated curves correspond to the simulation without the island. When the island model is on during $3.55-3.85 \mathrm{sec}$, an $\sim 25 \%$ deterioration is observed in the total neutron yield. Changes in the three components are indicated by the curves labeled with the symbols B-T for beam-target, B-B for beam-beam and T-T for target-target or thermal part. 
run \# 66868t11
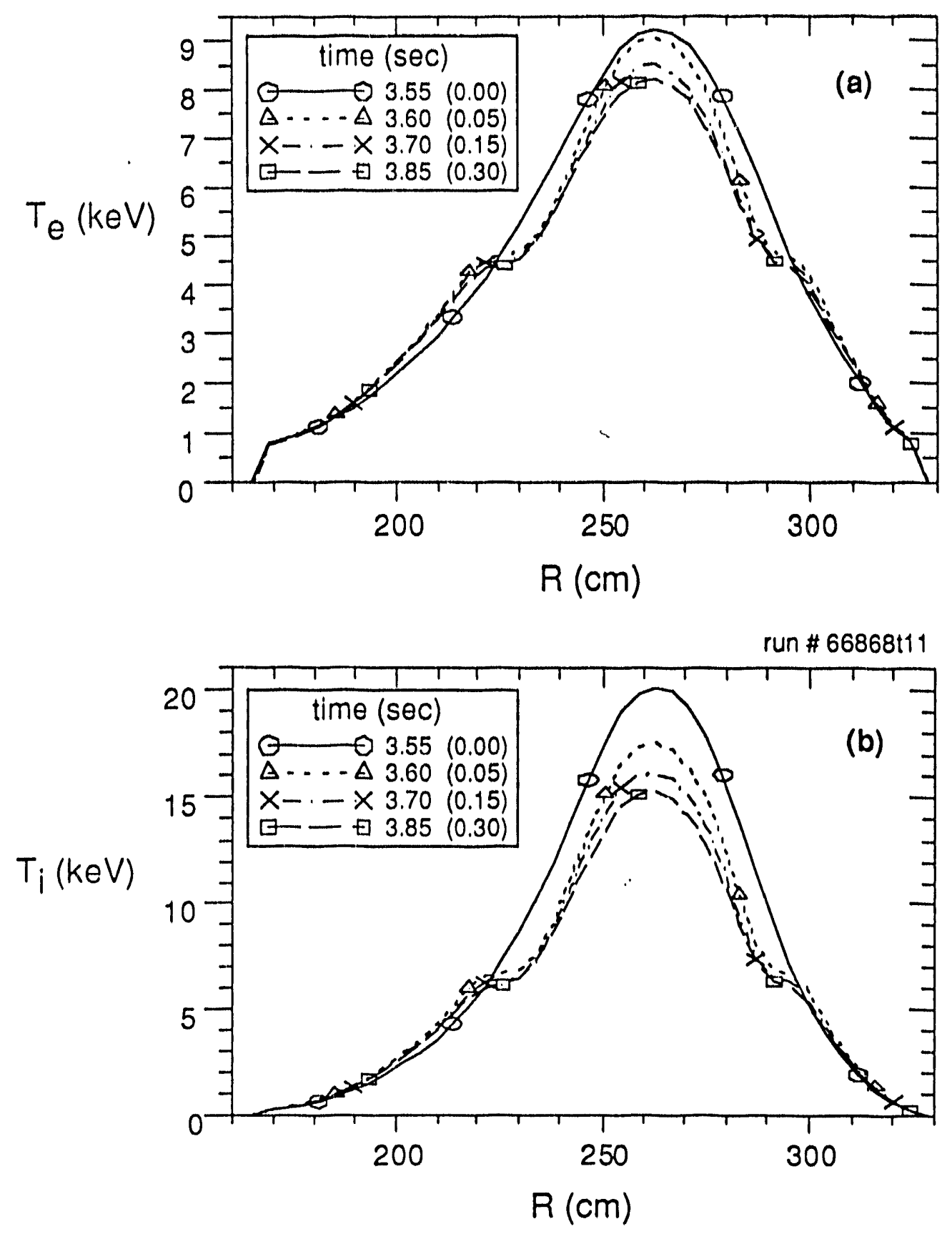

Figure 32: Time evolution of the TRANSP calculated (a) $T_{e}$ and (b) $T_{i}$ profiles after the onset of the island modesl. The time relative to the onset of the island is shown in the parenthesis. The $T_{e}$ evolution is very similar to the measurement shown in Fig. 17. A larger decrease in the central $T_{i}$ than the $T_{e}$ is observed, which is expected from the theory [see Eq. (4)] due to the larger ion temperature gradient around the mode rational surface. 


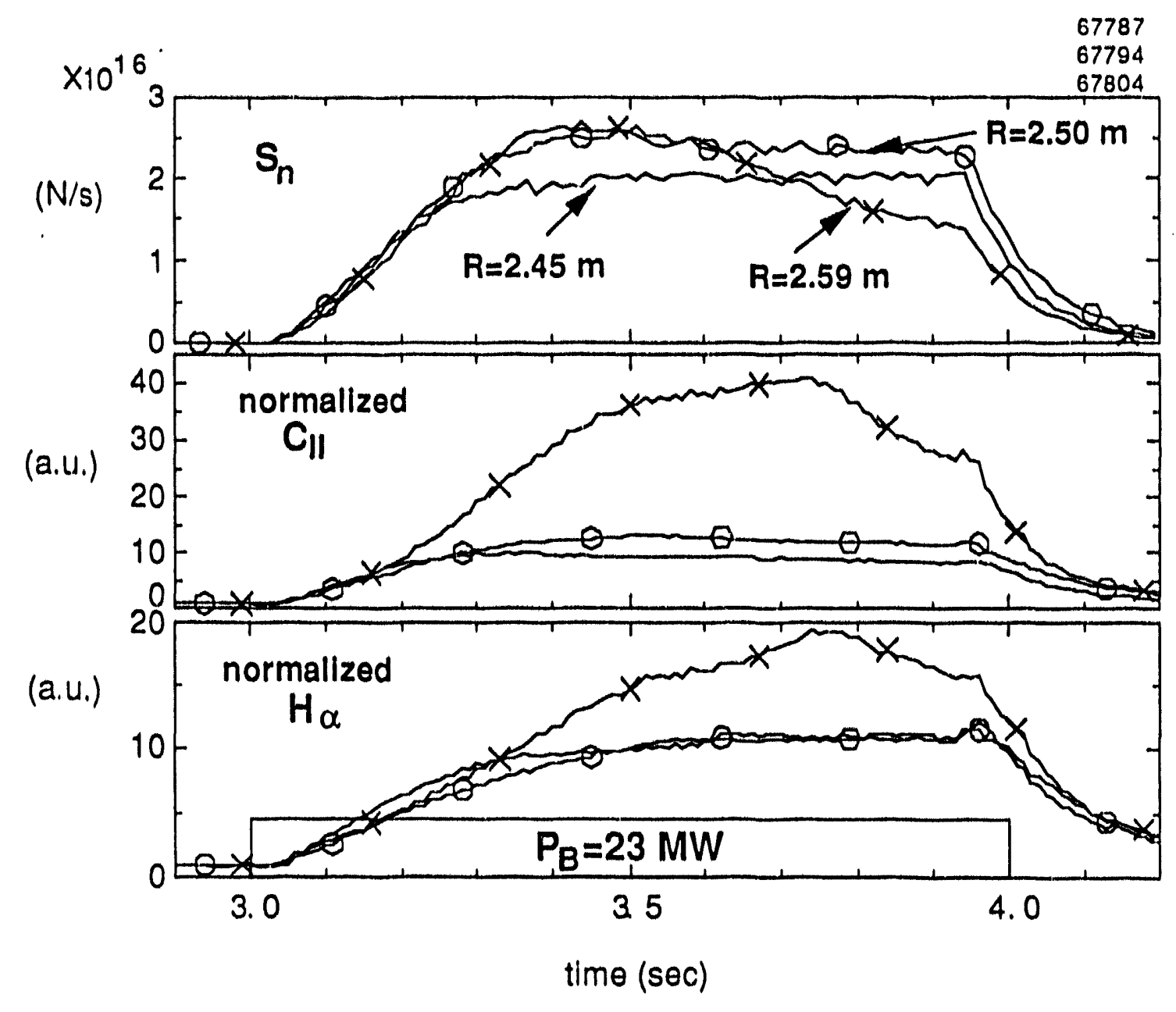

Figure 33: Comparison of three supershot discharges from a major radius scan experiment. They have the same neutral beam power, plasma current, target density, etc. The Carbon II and $H_{\alpha}$ data have been normalized to their values before the beam. 


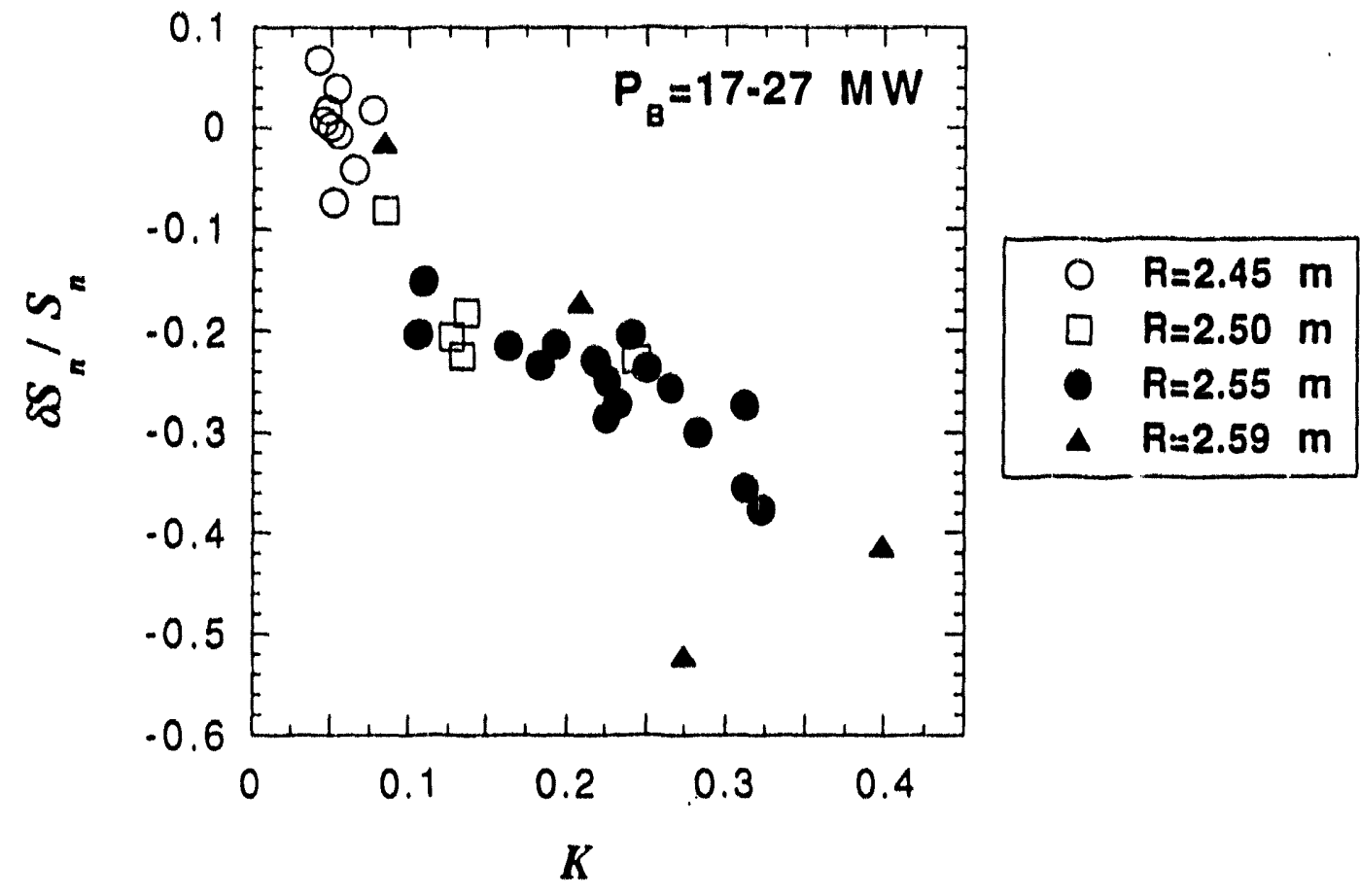

Figure 34: Correlation between the neutron deterioration and the "recycling parameter" $K$ defined in Eq. (18) for a major radius scan experiment. No strong coherent $m \geq 2$ modes are observed in these discharges. 


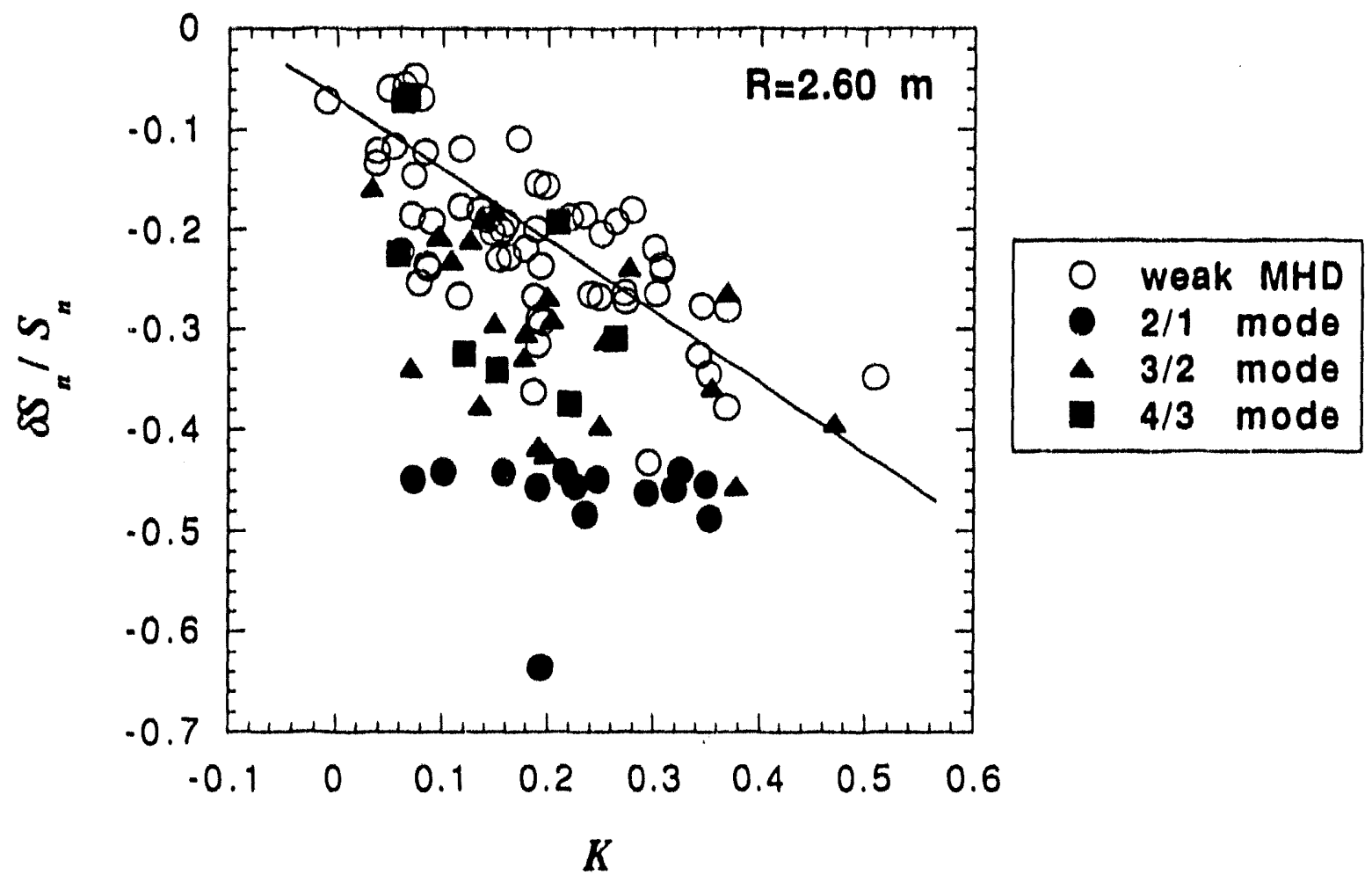

Figure 35: Similar statistical data analysis as Fig. 34 for a subset of $R \simeq 2.60$ $\mathrm{m}$ plasmas from the database used in Fig. 14. The straight line suggests the correlation tendency between the neutron yield deterioration and the recycling parameter $K$ for the weak MHD discharges. 
Dr. F. Peolon, Univ. of Wollongong, AUSTRALIA

Prol. M.H. Brennan, Univ. of Sydnoy, AUSTRaLIA

Plasma Rosearch Lob., Austrelian Nat. Univ., AUSTRALIA

Prol. I.A. Jones, Flinders Untr, AUSTRALIA

Prol. F. Cap, Inat for Theoretical Physics, AUSTRIA

Prot. M. Heindter, Insibul for Theoreveche Physik, AUSTRIA

Prol. M. Cooscens, Astronomisch IntituUt, BELGIUM

Ecole Roy de Mitisure, Lab. do Phy. Plaemas, BELGIUM

Commision-Europeen, DQ. XII.Fusion Prog., BELGIUM

Prol. R. Boudqua, Rifkeuniverdbet Gont, BELGIUM

Or. P.H. Sakanaka, Instituto Fisica, BRWZIL

Institu to Nadona Do Pesquieas Espadrir.INPE, BRUZIL

Documentu Offico, Abomic Enoroy ol Ceneda Ld., CANADA

Dr. M.P. Bectynak, MPB Technologies, Inc., CANADA

Dr. H.M. Skangard, Univ, of Sackatchowen, CANADA

Prof. J. Toichmam, Univ. of Montreal, CANADA

Prol. S.P. Sromivacen, Univ. of Calgary, CANADA

Prol. T.W. Jolmston, INRS-Energio, CANADA

Dr. R. Botton, Contre cansodien de tusion magnedique, CANADA

O. C.R. James, Univ. of Nborta, CANADA

Dr. P. Lukic, Komonaktho Univorszita, CZECHO-SLOVAKIA

The Librarian, Cutham Leborabry, ENGLAND

Librery. R61, Ruthertord Applaton Laboralory. ENGLAND

Mre. SA. Hutchinson, JET Libray, ENGLAND

Dr. S.C. Sheme, Univ, of South Pacific, FIJI ISLANDS

P. Mathonen, Univ. of Helsinkj, FINLAND

Prol. M.N. Buscenc, Ecolo Polybechnique,. FRANCE

C. Mountor, Leb de Physique dos Milioux lonises, FRANCE

J. Radil CENCADARUCHE - Bal 506, FRANCE

Prol. E Economou, Univ. of Crolo, GREECE

Ms. C. Rinni, Univ, of loannina, GREECE

Dr. T. Mud, Acadomy Bibliographic Sor., HONG KONG

Preprini Lubrary, Hungarian Acadamy of Sa., HUNGARY

Dr. B DasGupte, Saha Inst. of Nuctear Physics, INDIA

Dr. P. Kaw, Inst. tor Plasma Rosearch, INDIA

D. P. Rosenaw, Ieraed Inst of Technology, ISPAEL

Libranan, Intemational Conter lor Thoo Physics, ITALY

Miss C. Do Palo, Associaziono EURATOM.ENEA, ITALY

Dr. G. Grosso, Istuto di Fisica del Plasma, ITALY

Prol G. Rostengni, Istuto Gas tonizzat Del Cnr, ITALY

Dr. H. Yamato, Toshiba Ros \& Deral Contor, JAPAN
Prol. I. Kewakemi, Hroshima Univ., JAPAN

Prol. K. Nishikawa, Hroshima Univ., JAPAN

Orector, Japan Alomic Energy Research Inst., JAPAN

Prol. S. Ioh, Kyushu Univ., JAPAN

Recearch Into. Cr., Navonal Instit. for Fusion Science, JAPAN

Prol. S. Tenaka, Kyoto Univ., JAPAN

Librery, Kyolo Univ., JAPAN

Prol. N. Inow, Univ, of Tokyo, JAPAN

Socretary, Plasma Saction, Eloctrotechnical Lab., JAPAN

S. Mori, Tectriced Advicor, MAERI, JAPAN

Dr. O. Miterad, Kumamow Inst of Technotogy, JAPAN

J. Hycon-Sook, Koroa Abomic Energy Research Inst., KOREA

D.1. Chad, The Korea Adv. Inat of Sa. \& Toch., KOREA

Prot. B.S. Llley, Untw. of Weikato, NEW ZEALAND

Inst of Phyelac, Chinese Aced Sci PEOPLE'S REP. OF CHINA

Lborey, Inst of Plasma Physica, PEOPLE'S REP. OF CHINA

Tinghua Univ. Ubray, PEOPLE'S REPUBLIC OF CHINA

2. U. S.W. Inst Phyelas, PEOPLE'S REPUBLIC OF CHINA

Prol. J.A.C. Cabred, Inetiuto Superior Tocnico, PORTUGAL

Dr. O. Porrus, AL I CUzA Univ., ROMANIA

Dr. J. Villiors, Fusion Studios, AEC. S. AFAICA

Prot. M.A. Hellberg. Univ. of Natal, S. AFRICA

Prol. D.E. Kim, Pohang Inst. of Sai a Tech., SO. KOREA

Prof. C.I.E.M.A.T, Fusion Division Library, SPAIN

D. L SWAfio, Univ. Of UMEA, SWEDEN

Library, Royal Inst. of Tectnology, SWEDEN

Prol. H. Witholmeon, Chaimors Univ. of Toch., SWEDEN

Convo Phys. Des Plasmas. Ecole Polytech, SWITZERLAND

Bibliotheok, Inst. Voor Plasma.Fysica, THE NETHERLANDS

Asst Prot. Dr. S. Cekix, Modde Easl Tech Univ., TURKEY

Dr. V.A Gukthikh, Sa. Res. Inst. Eloctrophys. I Apparatus, USSR

D. D.D. Ayubr, Siberian Branch of Acadomy of Sai., USSR

Dr. G.A. Elicoov, I.V. Kurchatov Inst, USSA

Librarian, The Ukr.SSA Acadomy of Sciences, USSR

Dr. L.M. Kovizizimykh, Inst. ol General Physics, USSR

Kemlorsctungeaniege GmbH, Zentrabibliothok, W. GERMANY

Bibliothok, Inst. För Plasmatorsctiung. W. GERMANY

Prot. K. Schindler, Ruhr. Universital Bochum, W. GERMANY

Dr. F. Wagner, (ASDEX), Max.Planck-Institut, W. GERMANY

Litoravian, Max-Planck-Institut, W. GERMANY

Prol. R.K. Janow, Inst. of Ptyysics, YUGOSLAVIA 

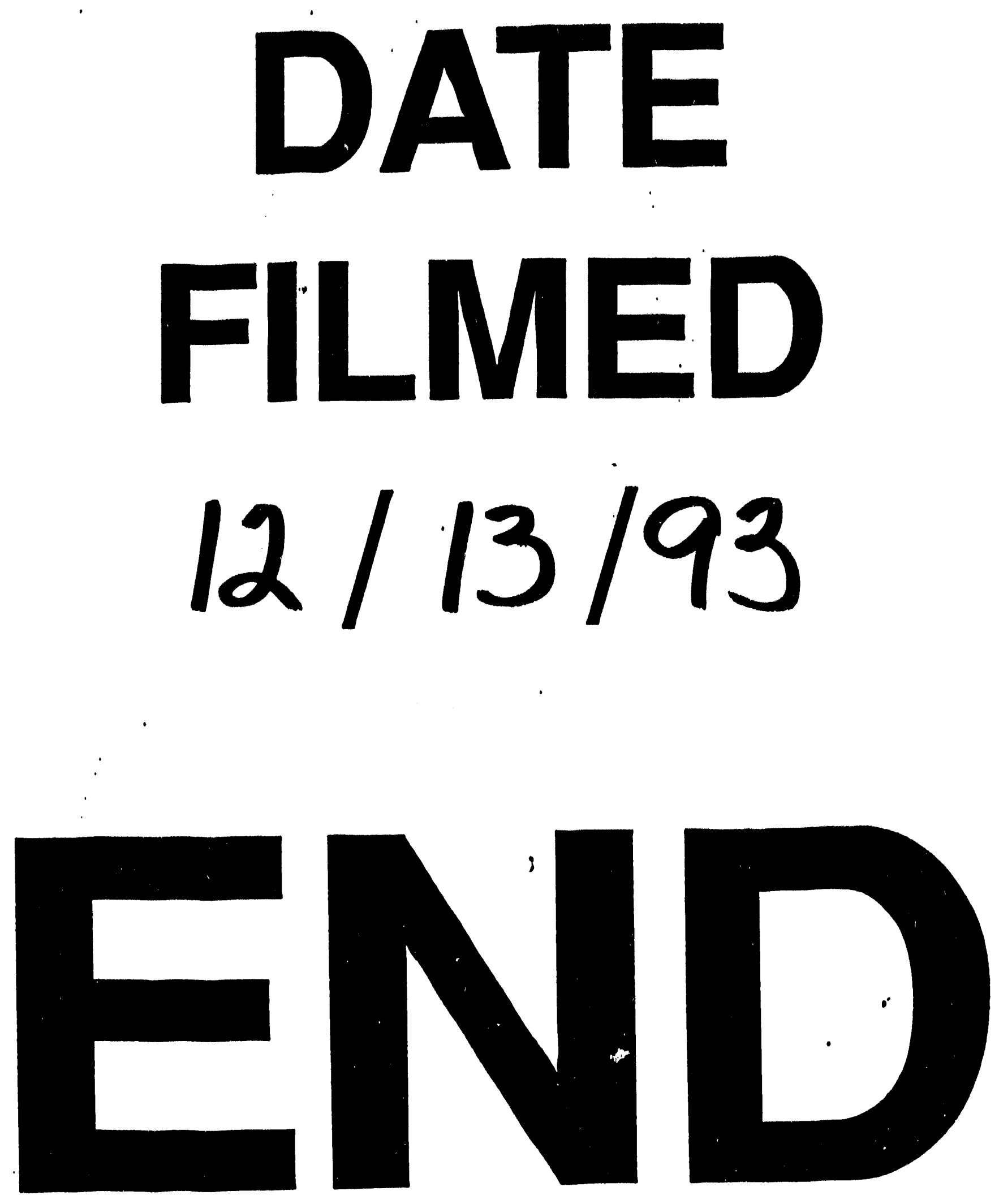